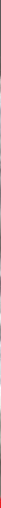

\title{
IntechOpen
}

\section{New Perspectives in Fluid Dynamics}

Edited by Chaoqun Liu

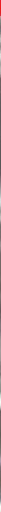





\section{NEW PERSPECTIVES IN FLUID DYNAMICS}

Edited by Chaoqun Liu 
New Perspectives in Fluid Dynamics

http://dx.doi.org/10.5772/59432

Edited by Chaoqun Liu

\section{Contributors}

Jorge Flores-Velazquez, José Simão Antunes Do Carmo, Daniel Marinho, Pedro Forte, Tiago Barbosa, Daniel Almeida Almeida Marinho, Medzid Muhasilovic, Chaoqun Liu

\section{(c) The Editor(s) and the Author(s) 2015}

The moral rights of the and the author(s) have been asserted.

All rights to the book as a whole are reserved by INTECH. The book as a whole (compilation) cannot be reproduced, distributed or used for commercial or non-commercial purposes without INTECH's written permission.

Enquiries concerning the use of the book should be directed to INTECH rights and permissions department (permissions@intechopen.com).

Violations are liable to prosecution under the governing Copyright Law.

\section{(cc) BY}

Individual chapters of this publication are distributed under the terms of the Creative Commons Attribution 3.0 Unported License which permits commercial use, distribution and reproduction of the individual chapters, provided the original author(s) and source publication are appropriately acknowledged. If so indicated, certain images may not be included under the Creative Commons license. In such cases users will need to obtain permission from the license holder to reproduce the material. More details and guidelines concerning content reuse and adaptation can be foundat http://www.intechopen.com/copyright-policy.html.

\section{Notice}

Statements and opinions expressed in the chapters are these of the individual contributors and not necessarily those of the editors or publisher. No responsibility is accepted for the accuracy of information contained in the published chapters. The publisher assumes no responsibility for any damage or injury to persons or property arising out of the use of any materials, instructions, methods or ideas contained in the book.

First published in Croatia, 2015 by INTECH d.o.o.

eBook (PDF) Published by IN TECH d.o.o.

Place and year of publication of eBook (PDF): Rijeka, 2019.

IntechOpen is the global imprint of IN TECH d.o.o.

Printed in Croatia

Legal deposit, Croatia: National and University Library in Zagreb

Additional hard and PDF copies can be obtained from orders@intechopen.com

New Perspectives in Fluid Dynamics

Edited by Chaoqun Liu

p. $\mathrm{cm}$.

ISBN 978-953-51-2228-9

eBook (PDF) ISBN 978-953-51-6644-3 


\section{We are IntechOpen, \\ the world's leading publisher of Open Access books}

Built by scientists, for scientists

\section{$3,800+$}

Open access books available

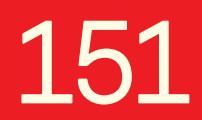

Countries delivered to

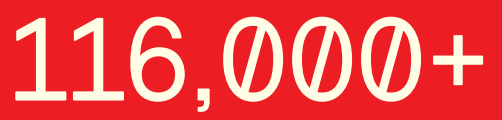

International authors and editors
$120 \mathrm{M}+$

Downloads

Our authors are among the

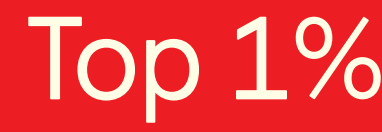

most cited scientists

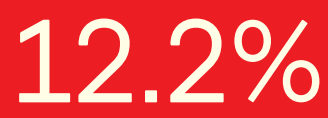

Contributors from top 500 universities

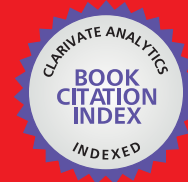

WEB OF SCIENCE ${ }^{\mathrm{TM}}$

Selection of our books indexed in the Book Citation Index in Web of Science ${ }^{\mathrm{TM}}$ Core Collection (BKCI)

Interested in publishing with us?

Contact book.department@intechopen.com

Numbers displayed above are based on latest data collected.

For more information visit www.intechopen.com

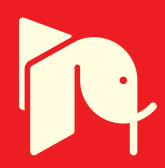





\section{Meet the editor}

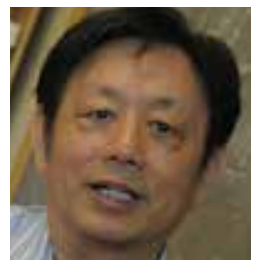

Dr. Chaoqun Liu received his bachelor of science degree in 1968 and master of science degree in 1981 from Tsinghua University, Beijing, China, and PhD in 1989 from the University of Colorado in Denver, Colorado, USA. He is currently a Distinguished Scholar Professor, the highest honored academic title, and Director of the Center for Numerical Simulation and Modeling at the University of Texas in Arlington, USA. He has been working in the areas of high-order direct numerical simulation (DNS) and large eddy simulation (LES) for flow transition and turbulence for 25 years since 1990. As PI, he has been awarded 47 federal research grants of over 5.7 million US dollars since 1990 by the United States. He has published 7 books, 86 journal papers, and 125 conference papers. As a principal lecturer, he conducted the TGS 2015 workshop on New Theory of Turbulence Generation and Sustenance at Tsinghua University, Beijing, China, on June 4-6, 2015, sponsored by 20 research institutions with over 220 audiences. He also chaired the first and the third AFOSR international conference on DNS/LES. He is an expert in high-order DNS/LES for flow transition, turbulence, and shock and boundary layer interaction. 



\section{Contents}

Preface XI

Chapter 1 Self-Contradictions of Current Turbulence Theory and Liu's New Turbulence Generation Theory 1

Chaoqun Liu and Shuhyi Chen

Chapter 2 Modeling of Wave Propagation from Arbitrary Depths to Shallow Waters - A Review 23

José Simão Antunes do Carmo

Chapter 3 CFD-Based Investigation of Wind-Strokes over Highway Bridge Section 67

Medzid Muhasilovic, Kenan Imsirpasic, Karel Ciahotny and Brano Sirok

Chapter 4 Computational Fluid Dynamics Achievements Applied to Optimal Crop Production in a Greenhouse 77

Jorge Flores-Velázquez, Abraham Rojano, Adriana Rojas-Rishor and Waldo Ojeda Bustamante

Chapter 5 Technologic Appliance and Performance Concerns in Wheelchair Racing - Helping Paralympic Athletes to Excel 101 Pedro Forte, Tiago M. Barbosa and Daniel A. Marinho 



\section{Preface}

Although fluid dynamics, a classical discipline, has been studied for many centuries, there are many questions still that remain unanswered, especially on turbulent flow. With significant advances in computational technology and decrease in hardware costs, a rapid evolution in computational fluid dynamics (CFD) techniques and models has been noticed. Many industrial engineering sectors are also making a concerted effort in the development and advancement of fluid dynamics. With the increasing demand for research, I believe the future for the advancement of fluid dynamics could not be brighter. Thus, the purpose of this book is to invite the leading researchers engaged in various research areas, relating to fluid dynamics, to come together and present their recent achievements and invoke further discussions. This book will mainly focus on the individual expert's experiences and discoveries. Although we realize that a single chapter with selected topics cannot provide exhaustive representations, it should give readers great insight into experts' accomplishments.

This book contains five chapters. The new turbulence theory and the advances and wide applications of CFD are described in each of the chapters as follows.

In the first chapter, "Self-Contradictions of Current Turbulence Theory and Liu's New Turbulence Generation Theory", Dr. Liu and Dr. Chern give a critical review of the classical and current turbulence theories and point out many self-contradictions existing in the classical and current turbulence theories. In this chapter, the authors provide more than 10 topics about the misconceptions created of the classical and current turbulence theories, especially baffling with two totally different concepts, vortex and vorticity tubes. Turbulence is one of the most important but unresolved problems in the modern fluid dynamics. Mathematically, one counter example can overthrow a theory without the need of a second example. However, classical and current turbulence theories are not only filled with one but many self-contradictions. Therefore, we really do not have the right turbulence theory. The transition community believes that turbulence is generated by "vortex breakdown,", but the turbulence community believes that there are coherent structures in the fully developed turbulence after vortex breakdown. In other words, studying the structure of a house after its collapse is meaningless. There are many other such contradictions. This chapter introduces a new turbulence generation theory given by Liu. According to Liu, (i) flow transition is not a process of vortex breakdown but turbulence vortex structure buildup; (ii) the nature of turbulence generation is that fluids cannot tolerate high shear, and shear must be transferred to the rotation and form a very fast rotation core; (iii) "shear layer instability" is the "mother of turbulence"; (iv) small-scale turbulence is generated by multi-level shear layers that are generated by multi-level sweeps, ejections, and negative and positive spikes; and (v) a large vortex provides energy to smaller vortices through fast rotation, which causes strong sweeps. According to Liu, the nature of the flow transition is mainly due to vorticity roll-up 
from the wall. The flow transition is vorticity redistribution and increment from near wall to whole boundary layer. In addition, flow transition is a process of non-rotational vorticity transferring to rotational vorticity.

In the second chapter, "Modeling of Wave Propagation from Arbitrary Depths to Shallow Waters: A Review", José Simão Antunes do Carmo points out that numerical models are a useful instrument for studying the complex superposition of wave-wave and wave-current interactions in coastal and estuarine regions and investigating the interaction of waves with complex bathymetries or structures built in nearshore areas. Moreover, since their applications are significantly less expensive and more flexible than the construction of physical models, they form a convenient tool to support design. The ability of the standard Boussinesq and Serre or Green and Naghdi equations to reproduce these nonlinear processes is well known. However, these models are restricted to shallow water conditions, and the addition of other terms of dispersive origin has been considered since the 1990s, particularly for approximations of the Boussinesq type. To allow applications covering a greater range of area, other than shallow waters, (where the water depth is at rest and so is the wavelength), a new set of Serre-type equations, with additional terms of dispersive origin, is developed and tested with the available data and with a numerical solution of a Boussinesq-type model, also improved with dispersive characteristics. Explicit and implicit methods of finite difference are implemented to solve both approximations of Boussinesq and Serre- types with improved dispersive characteristics. A finite element method is also implemented to solve an extension of a Boussinesq-type model that takes into account the wave-current interactions. Application examples to solve real-world problems are shown and discussed. The performances of $1 \mathrm{HD}$ models are compared with experimental data of very demanding applications, namely (i) a highly nonlinear solitary wave propagating up a slope and reflecting from a vertical wall and (ii) a periodic wave propagating in intermediate-depth waters upstream a trapezoidal bar, followed by very shallow waters over the bar, and again in intermediate-depth water conditions downstream.

In the third chapter, "CFD-Based Investigation of Wind-Strokes over Highway Bridge Section”, Dr. Medzid Muhasilovic (M.Sc.), Kenan Imsirpasic (M.Sc), Prof. Dr. Karel Ciahotny, and Prof. Dr. Brano Sirok point out that there is an almost everlasting debate on the possibilities of investigation tools for their applications in a prospective fashion, while solving the engineering tasks, and against the fact that there is use of these engineering tools, while correcting the existing technological problems. A unique chance to compare these tools of research in traffic intentions, while setting the modern road communications through southern Bosnia and Herzegovina, with the natural occurrences in the atmosphere (such as a strong north wind in this geographic region), offers the highway section of Pocitelj-Zvirovici. In such cases (and before the actual construction of this highway bridge), "for the sake" of prospective engineering, the CFD mechanism (the "tool kit" for performing computational fluid dynamics) was applied to solve this atmospheric problem. Both in steady-state explorations (while applying the k- $\varepsilon$ turbulence treatment) and in the time-dependent CFDbased mode, the authors explored the wind strokes at $10 \mathrm{~m} / \mathrm{s}, 20 \mathrm{~m} / \mathrm{s}, 30 \mathrm{~m} / \mathrm{s}$, and $40 \mathrm{~m} / \mathrm{s}$, an expected but certainly unwanted strong gaseous flows over the bridge, detecting the traffic safety edge points. Results obtained from the explorations of the CFD tool are explained and discussed in this chapter.

In the fourth chapter, "Computational Fluid Dynamics Achievements Applied to Optimal Crop Production in a Greenhouse", Jorge Flores-Velázquez, Abraham Rojano, Adriana Ro- 
jas-Rishor, and Waldo Ojeda Bustamante point out that computational fluid dynamics (CFD) has been successfully used in protected agriculture to simulate greenhouse weather as a physical process. The variables involved are velocity, wind direction related to absolute or relative humidity, temperature, as well as deficit vapor pressure and carbon dioxide, among others. The research evolution is changing from the traditional validation of new designs and management to the testing of efficient production with less environmental pollution. This study points out the assessment based on the physical principles of conservation of mass, momentum, and energy. Constitutive relationships like Darcy-Forchheimer porosity model in the momentum equation as well as the geometrical and physical properties of the materials involved are needed to fulfill particular issues of temperature, wind, and humidity. As a result, this study is enhanced by the effect of solar radiation in processes such as crop transpiration with dynamical meshes and condensation. Most of the processes that occur in a greenhouse are related to mass transfer of events (relative humidity and transpiration), momentum (velocity and species), and energy or exchange of heat (convection and radiation), and consequently the computational fluid dynamics is a robust tool for greenhouses and animal farm behavior simulations. The inclusion of culture and auxiliary climatic control systems is essential to the production process from an agricultural point of view. The results of this study indicate that once the models are experimentally validated, the use of these systems allows to know the spatial characteristics of the variables involved. From a mechanical point of view, the use of anti-insect screens and crop presence cause a drop of $80 \%$ in speed. A heating system for hot pipes under the atmospheric conditions of central Mexico is only necessary for specific periods before sunrise. A change in the position of the greenhouse windows in zigzag shape increases air movement and homogenized conditions inside, even if the same ventilation ratio is maintained, but a variation in the porosity of the mesh has side effects in the air renewal ratio. The prospect of computational fluid dynamics applied to the protected agriculture will focus on building a dynamic model with many variables to make an efficient use of resources and reduce the use of fossil fuels in crop production.

In the last chapter, "Technologic Appliance and Performance Concerns in Wheelchair Racing: Helping Paralympic Athletes to Excel,", Pedro Forte, Tiago M. Barbosa, and Daniel A. Marinho show that numerical simulations have provided useful evidence on helping several sportsmen to excel. This methodology enables a deeper understanding of the influence of equipment and sports techniques on sports performance. In wheelchair racing, technology was used without considering Olympic sports (once some of the Paralympics used the same technology of their Olympic counterparts) and induced unique changes in prosthetic and wheelchair devices. Eventually, technology has become an essential part of Paralympic sports, wheelchair racing being one of the most popular events. Numerical simulations can help us gather evidence on the effects of drag force, acting upon the athlete-chair system. Different types of wheelchairs are designed for racing (track and road races) and net and invasion sports. One of the various strategies to enhance performance is to minimize the aerodynamic drag of the frame, tires, helmet, sports outfit, and body posture. Numerical simulations can be used to predict fluid dynamics. The aim of this chapter is to review the state of the art on numerical simulations in wheelchair racing and suggest further studies. The chapter includes sections covering (i) the main determinants in wheelchair racing, (ii) the effect of aerodynamic force on performance in wheelchair racing, (iii) analytical models, experimental testing, and numerical simulations in wheelchair racing, (iv) numerical simulations on equipment and techniques. The wheelchair racing sports sciences lack research; 
however, there is some information that could positively contribute to the performance increase in the athletes. While managing the mathematical models based on physics laws, coaches could identify the mechanical performance obstacles and try to minimize them. The drag reduction by the rolling resistance and air resistance access also contributes to the performance increase in the athletes. A better propulsion could also be obtained by reducing the total drag associated to a mass reduction and other possible aerodynamic positions. The purpose of the aerodynamic drag reduction in computational simulations and/or computer fluid dynamics should be an improvement in the winning time. No data pertaining to CFD tests in wheelchair racing were found. The stroke technique should also be focused on despite of the fact that there are indications on the number of hours of training that should be taken for an injury occurrence. It is stated that high angular velocities near the shoulder and the elbow, generated by the strokes, induce stress, increasing the risk for joint injuries. There are also some indications about the contact zones, force applied, and contact break at the rear wheels. Physiological variables should also be considered in the wheelchair racing athletes, which certainly lacks information regarding this sport. Thus, it is acceptable to say that a precise control of the total drag and an efficient use of the stroke technique allied to high levels of strength derived from the strength and physical training conditions will positively contribute to a better performance in the athletes.

I hope this book will be useful to scientists and engineers who are interested in fundamental fluid dynamics and its applications in engineering.

In conclusion, I would like to thank all the authors for their incredible contributions to this book and their patience in assisting us. Furthermore, I would like to acknowledge and thank the referees for their tiresome effort in making this book come to fruition. Last but not the least, I would like to thank my family, including Weilan Jin (my wife), Haiyan Liu (my daughter), and Haifeng Liu (my son), for their unconditional support.

Chaoqun Liu

Distinguished Scholar Professor University of Texas at Arlington

Arlington, USA 
Chapter 1

\title{
Self-Contradictions of Current Turbulence Theory and Liu's New Turbulence Generation Theory
}

\author{
Chaoqun Liu and Shuhyi Chen \\ Additional information is available at the end of the chapter \\ http://dx.doi.org/10.5772/61836
}

\begin{abstract}
Turbulence is one of the most important but unresolved problems in modern fluid dynamics. Mathematically, one counterexample can overthrow a theory and we really do not need a second example. However, classical and current turbulence theories are filled with not only one but many self-contradictions. Therefore, we really do not have the right turbulence theory. The transition community believes that turbulence is generated by "vortex breakdown," but the turbulence community believes there are coherent structures in fully developed turbulence after vortex breakdown. It is equivalent to say we study the structure of a house after the house collapses and breaks down. This is really ridiculous. There are many others. According to Liu, (1) flow transition is not a process of vortex breakdown but turbulence vortex structure buildup; (2) the nature of turbulence generation is that fluids cannot tolerate high shear and shear must transfer to rotation and form a very fast rotation core; (3) "shear layer instability" is the "mother of turbulence"; (4) turbulence small scales are generated by multiple-level shear layers that are generated by multiple level sweeps, ejections, negative and positive spikes; (5) large vortex provides energy to smaller vortices through fast rotation, which causes strong sweeps. According to Liu, the nature of the flow transition is mainly caused by vorticity rollup from the wall. Flow transition is vorticity redistribution and increment from near wall to whole boundary layer. In addition, flow transition is a process of non-rotational vorticity transferring to rotational vorticity.
\end{abstract}

Keywords: Vorticity, vortex, rotation, vortex buildup, shear layer, boundary layer transition

\section{Introduction}

Turbulence is still an unsolved scientific problem, which is not only important to science but also to industrial applications in aerospace engineering, mechanical engineering, energy 
engineering, bioengineering, and many others. Turbulence remains the most important unsolved problem of classical physics. Clearly, understanding of turbulence will help scientists and engineers cope with the broad range of turbulent flows. Nobel Prize winner Richard Feynman considered turbulence as "One of the most important unsolved problems of classical physics" [34]. Nobel Prize winner Werner Heisenberg said, "When I meet God, I am going to ask him two questions: why relativity? And why turbulence? I really believe he will have an answer for the first" [29]. These comments and addresses clearly show turbulence remains a top secret in nature and awaits more research activities.

Mathematically, one counterexample can overthrow a theory and we really do not need a second example. However, classical and current turbulence theories are filled with many selfcontradictions. Therefore, we really do not have the right turbulence theory. The transition community believes that turbulence is generated by "vortex breakdown," but the turbulence community believes there are coherent structures in fully developed turbulence after vortex breakdown. It is equivalent to say we study the structure of a house after the house collapses and breaks down. This is really ridiculous. According to Liu, flow transition is not a process of vortex breakdown but turbulence vortex structure buildup.

In the current turbulence textbooks, there are many places filled with self-contradictions. We first say vortex never breaks down according to Helmholtz vorticity flux conservation law, and later we say turbulence is generated by "vortex breakdown." We first say vortex can only end on the wall surface and later say vortex can detach from the wall surface. How can a vortex attach to a wall, and then become detached, break down, and reconnect? These will leave vortex leg inside the flow field and directly violate vorticity flux conservation law.

Some people argue that "we do not have exact definition for vortex." If we really have no definition for "vortex," we then have no serious scientific research at all for turbulence study. In fact, the definition of a "vortex" is clear, which is a fluid rotation core but not vortex tube. Our textbooks say lambda vortex becomes hairpin vortex through "self-deform," but there should not be any "self-deform" in the world. "Deformation" is a motion and any motion must be driven by force. Some literatures say multiple vortex rings are auto-generated. However, the world should not have anything to be auto-generated. Everything in this world should be generated under certain mechanism.

Many people accepted the concept of "turbulence bursting" and "turbulence intermittency." However, this is a misunderstanding. Turbulence is generated by very organized activities, step by step. There is no possibility that the turbulence could suddenly appear and then suddenly disappear. It would be a question that if God manipulates the fluid flow as a result of that turbulence could suddenly appear and then suddenly disappear? This is a misunderstanding by some people who do not fully understand turbulence.

Richardson [31] believed there is an eddy cascade, but no one was able to find such a vortex cascade, even today when the 3-D PIV and laser equipment is quite advanced. Kolmogorov [11] believed the larger vortex gives energy to smaller vortices through "vortex breakdown," but there is no "vortex breakdown" in this world. Especially, no matter how one defines "vortex," turbulence has no way of being generated by "vortex breakdown." 
Linear mode suppression technology was studied for decades for flow transition control, and tens of million dollars have been spent in US and Europe, but who succeeded? When the perturbation is larger than $2 \%$, there are no linear modes. If flow transition was caused by linear modes and must experience the process of self-deform from lambda vortex to hairpin vortex, how could we explain "bypass transition" and "free stream turbulence"? Anyway, the classical and current turbulence theories are full of self-contradictions.

There are many others. This paper tries to revisit the classical and current turbulence theory, find some self-contradictions, and briefly introduces Liu's new turbulence theory.

After 25 years of intensive study by Dr. Chaoqun Liu and his students at the University of Texas at Arlington, Dr. Liu presented a new theory on turbulence generation and sustenance, which is consistent and has no self-contradictions. Compared with the current theories on turbulence, which is mainly empirical and derived on the basis of dimensional analysis, Liu's theory is based on accurate scientific computing and experiments. Therefore, the new theory is more trustworthy. The core of Liu's theory maintains that the nature of turbulence is such that shear must transfer to fast rotation cores in a flow field and that turbulence is not generated by vortex breakdown, but multiple level shear layer instability, which is generated by sweeps and ejections. "Shear layer instability is the mother of turbulence," and "it is the nature of turbulence that shear must transfer to fast rotation." Turbulence is not generated by "vortex breakdown" but "vortex buildup." This new theory may bring a revolution to not only the basic fluid mechanics, but also to practical engineering applications including turbulence modeling.

$\mathrm{Wu}$ and Moin [36] reported a new DNS for late flow transition on a flat plate. They obtained fully developed turbulent flow with structure of a forest of hair-ping vortices by flow transition at zero pressure gradients. However, they did not give the mechanism of the late flow transition. Actually, similar work for the whole process of K- and H-type transition has been reported by Liu et al. [12-14] 14 years ago, and Rist et al. [32] 7 years ago. The newer results have higher resolution, but all reported vortex structures are similar.

In order to arrive at a deeper understanding of the physics of turbulence generation and sustenance, we recently conducted a high-order direct numerical simulation (DNS) with $1920 \times 241 \times 128$ gird points and about 600,000 time steps to study the mechanism of the late stages of flow transition in a boundary layer at a free stream Mach number 0.5 [2-6, 12-23, 24-28, 37-38]. The DNS results have been well-validated by UTA and NASA Langley researchers [10]. A visualization method combining $\lambda_{2}$ iso-surface [9] and vortex lines were used to describe the flow field.

According to the current flow transition theory, the flow transition process has been described as follows: (1) receptivity, (2) linear instability, (3) nonlinear growth and interaction, and (4) breakdown to turbulence. However, the authors believe that turbulence is not caused by "vortex breakdown" but "vortex buildup" and linear modes only play a role in triggering vorticity rollup, but not directly causing the flow transition. Therefore, the authors believe that the transition process should be described as follows: (1) perturbation and growth (which may include linear modes or other disturbances); (2) large vortex formation including vorticity 
rollup and shear layer instability; (3) multiple-level vortex structure buildup including sweeps, ejections, and small length scale generation; and (4) symmetry loss and being chaotic to turbulence [23].

First, contrary to current transition theory, there is no "vortex breakdown" but there is a "turbulence vortex structure buildup," which is just the opposite. "Vortex breakdown" is theoretically incorrect and is never observed by any experiment or DNS. At present, most flow transition papers just use one term, "vortex breakdown," to describe the last stage of flow transition. If "vortex breakdown," which never exists, means flow transition from the laminar state to turbulence state, the authors believe that we will need more than one hundred research papers to describe such a process, not just the one term, "vortex breakdown." According to Dr. Cai's high-resolution experiment (Figure 1, personal communication) with the highest resolution of $1 \mu \mathrm{m}$, while most of our experiments only have $\mathrm{mm}$-scale resolution, large vortices interaction could produce countless small vortices with the scale in the order of $1 \mu \mathrm{m}$ but did not find any large vortex breakdown, even no large vortex deformation. The large vortices are still alive, which contradicts to Richardson's large vortex short turnover time, expected by $l / u$ [7]. Cai et al conclude that the classical and current theory that small length vortices are produced by large vortex breakdown and that the energy is passed from large vortex to smaller vortex through "vortex breakdown" has no way to be correct. The other impressive qualitative agreement is that the vortex rings rotate fast with a rotation speed of around 10,000 circles per second in a jet flow, while our DNS shows that the rotation speed is around 8,000 circles per second in a boundary layer. According to the DNS observations by Liu, no small scales survive if the large vortex disappears, since the small scales are generated and supported by the large vortex structure.

Mathematically, one counterexample can overthrow a theory and we really do not need a second example. The classical and current flow transition theories are filled with many misunderstandings and self-contradictions. Thus, we do not have the right turbulence theory. The classical and current flow transition and turbulence theories must be revisited.

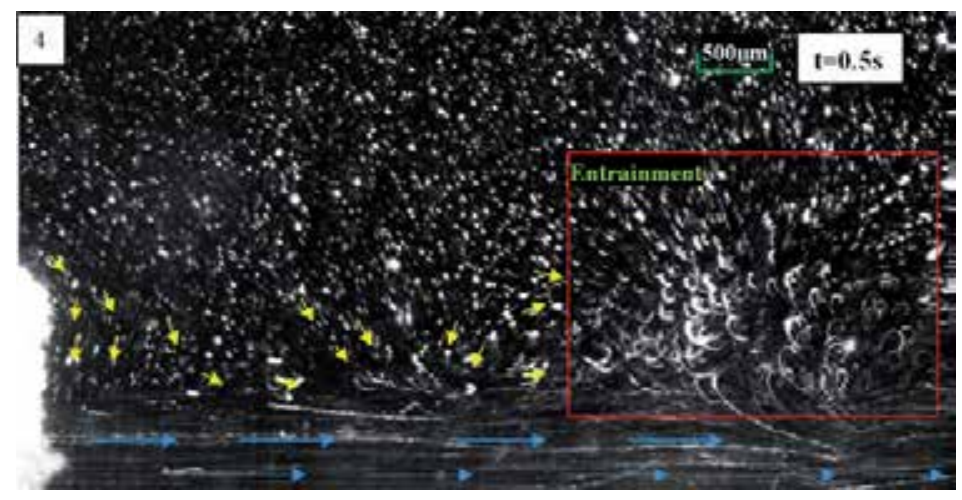

Figure 1. Vortices generated by water jet (Cai's experimental observation with highest resolution of $1 \mu \mathrm{m}$; personal communication) 


\section{Self-contradictions of classical and current turbulence theories}

As shown below, the classical and current turbulence theories have many serious selfcontradictions:

1. Vortex breakdown: The transition community commonly agrees that after receptivity, linear instability and nonlinear instability stages, flow will "break down" to turbulence, which means the turbulent flow does not have structure at all due to the "breakdown." However, the turbulence community believes there are coherent structures in fully developed turbulence after the flow breakdown. It does not make much sense to study the house structure after the house collapses and breaks down into hundreds pieces of debris. If turbulence has coherent structure, the flow structure must never break down during the transition. This question must be answered for any serious scientific research on turbulence and it cannot be skipped or ignored. Liu et al. [23] believe there is no breakdown in any sense and both transitional flow and turbulent flow have the same mechanism for turbulence generation and sustenance and certain structure that can be accepted by Navier-Stokes equations. The fully developed turbulent flow has more small structures in a more chaotic manner. Liu et al. [23] also believe that when the transition community discusses the flow transition process and the turbulence community discusses the turbulence "coherent structure," they really discuss the same thing, which is "turbulence generation and sustenance" and should have the same mechanism. According to the authors, flow transition is not a process of vortex "breakdown," but just the opposite which is a process of turbulence vortex structure "buildup." There may be some arguments that "breakdown" does not mean one vortex breaks to hundreds of pieces. However, no one was able to observe even that one breaks into two pieces, like that shown in many textbooks for Richardson's eddy cascade and Kolmgorov's vortex breakdown (e.g., [7]) In any case, one cannot believe the English word "breakdown" means "buildup," or house "breakdown" means we are building the house. Flow transition is a process of vortex "buildup." Some people may argue that we do not have the exact definition for vortex. Actually, if we define vortex as a vortex tube, vortex tube cannot break down. If we define vortex as a rotation core, the core is very stable and cannot break down either, like a tornado. However, if we have no definition for vortex, we then have no serious scientific research on turbulence. The definition of vortex is apparently a rotation dominant flow with a rotation core which has less dissipation. In practice, vortex breakdown is mainly caused by inappropriate pickup of lambda 2 or Q-iso-surface.

2. The role of linearly unstable modes: Although the linear modes are well-understood, Liu et al. [23] believe that the modes may be still linear only when its magnitude is smaller than $2 \%$. When the perturbation is greater than $2 \%$, there are no linear modes at all since the base flow has been changed. How can we still find T-S modes when we do not have Blasius base flow? The authors' DNS shows we do not have Blasius velocity profile at very early transition stage and the inflection points are developed as the vorticity rolls up. The significant perturbation growth and vortex structure formation are all nonlinear. The flight environment cannot keep the inflow perturbation to be smaller than $2 \%$. Further 
study found that although the linear unstable modes are important, they are small, cannot form vortex, and cannot cause the flow transition either by absolute instability, convectional instability, or mode interaction. The complex turbulence structure cannot be formed by those mode interactions or resonance, but sophistic vortex development, step-by-step [23]. Actually, the flow transition is vorticity redistribution and increment and the vorticity is given by the original Blasius solution for the flat plate case. Actually, the linearized N-S solution departs from DNS solution at very early stages (Figure 2)

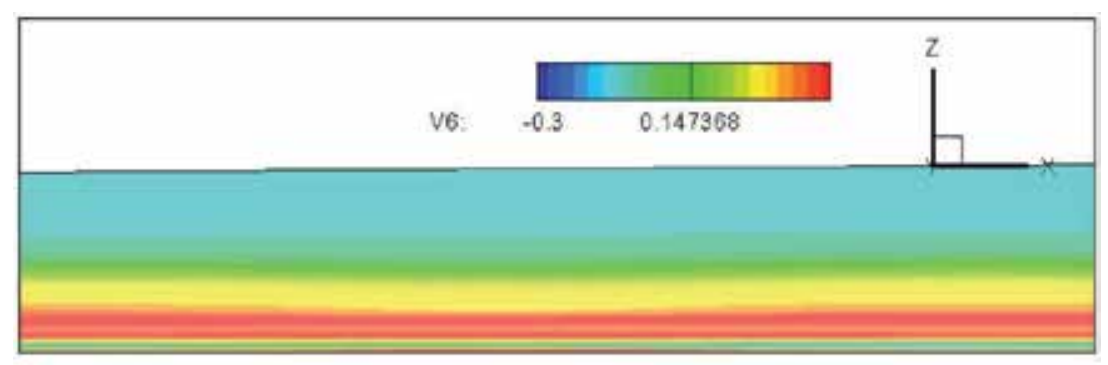

(a) Linearized N-S solution

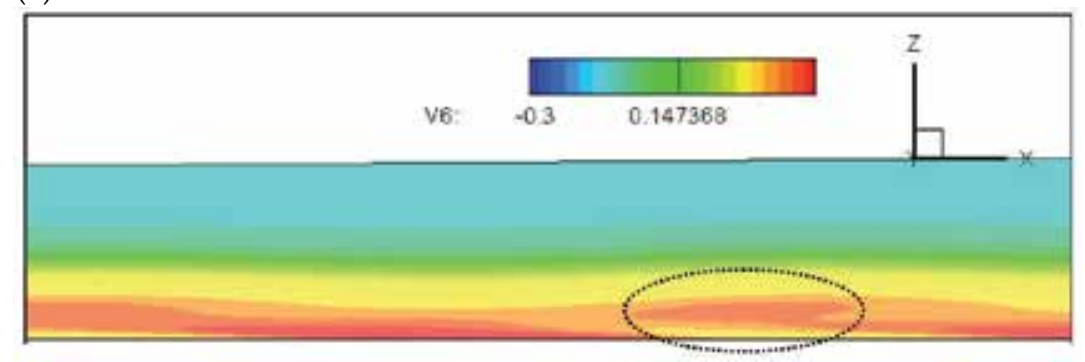

(b) DNS solution

Figure 2. Comparison of linearized solution and DNS solution at early stage of flow transition

3. Nature of turbulence: Liu et al. [23] believe that the flow transition is caused by the flow inherent property that fluid cannot tolerate high shear, and shear must transfer to rotation. The role of perturbation (linear modes or others) is to trigger vorticity rollup, which leads to flow transition. Similar to linear modes, any perturbation, like gust, dust, sands, mosquito, fly, roughness, blowing, can trigger the vorticity rollup and then cause the transition. Most transition researchers believe turbulence is caused by unstable modes growth and their interaction or resonance to vortex breakdown. However, that is not the case and how can vortex break down like resonance to produce turbulence with coherent structure? There are some arguments that we have no exact definition on "vortex" and "breakdown." However, no matter how one defines "vortex," there is no possibility that turbulence is generated by "vortex breakdown." 
4. $\Lambda$-vortex to hairpin vortex: Hama et al. [8] and Moin et al. [30] believe the $\Lambda$-vortex becomes hairpin vortex through a self-deformation mechanism. First, there must be no self-deformation. Deformation is a motion and any motion must be driven by some force and cannot be "self-deformed." Liu et al. [23] believe $\Lambda$-vortex root and vortex ring are generated separately and independently through different mechanisms and vortex ring is not part of $\Lambda$-vortex. Correctly understanding the hairpin vortex formation is key to understanding turbulence. According to Liu et al., the hairpin vortex has ring and legs. The legs are generated by vorticity rollup, which can generate low speed zones above the legs through vortex rotation (ejections), and the ring is generated by shear layer instability (K-H type.) In many turbulence textbooks (e.g., [7]) $\Lambda$-vortex is defined as a vortex tube. The authors believes it is a serious misunderstanding that vortex is defined as a vorticity tube without vorticity line leakage, like stream tube; $\Lambda$-vortex is a rotation core which is open for vorticity lines to come in and come out (Figure 3).

5. Vortex reconnection: Based on current theory, since the hairpin vortex tube (see [7]) has to be stretched, it will have to break down as the leg is placed on the wall surface where velocity is zero and the ring head is located almost near the inviscid area where the streamwise velocity is near one unit (the dimensional speed could be $170 \mathrm{~m} / \mathrm{s}$ if $\mathrm{M}=0.5$.) Some literatures suggest the hairpin vortex will break down and reconnect. The vortex tube breakdown concept directly violates the Helmholtz vorticity flux conservation law, which states that vortex tube foot must lie on the boundary and cannot lie down inside the flow field. There is no mechanism to support either vortex breakdown or reconnection. Liu et al. believe it is a serious mistake to consider "vortex" as a "vortex tube" (e.g., Figure 4 in the book, Turbulence, Davidson, 2004) and consider "vortex" as a congregation of vorticity lines with a rotation core, but, in general, is not a vortex tube. Actually, we never have vortex tube which is laid on the wall and vortex is never attached to the wall.
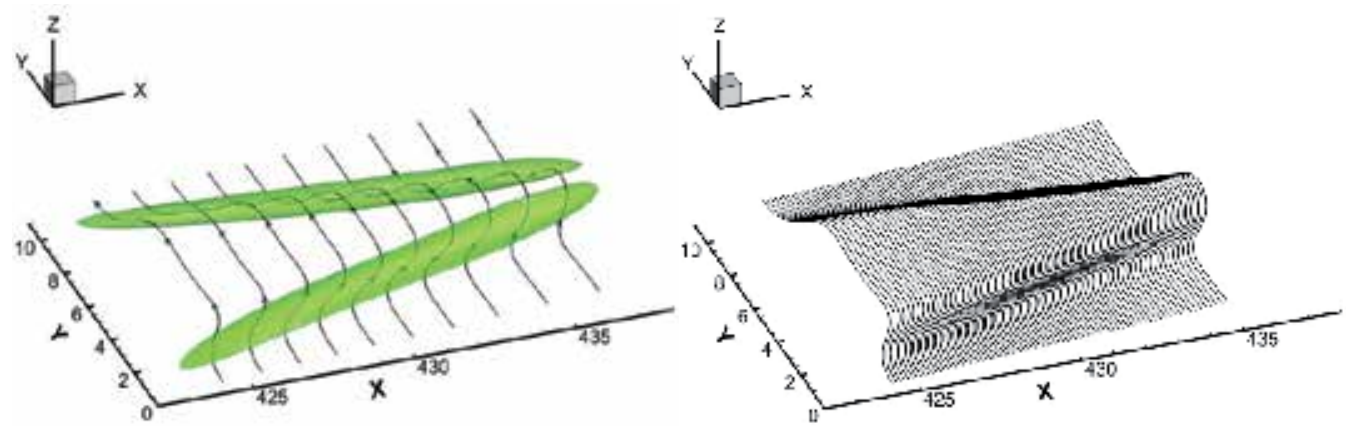

Figure 3. $\Lambda$-vortex (green part) is not a vortex tube as many vortex filaments (solid lines) penetrate the vortex

6. Vortex attachment and detachment: Some literatures suggest that the vortex is originally attached on the wall and then detached from the wall. It is really hard to believe how the vortex leg is originally linked with the wall surface and then detached from the wall. Liu et al. [23] believe the vortex (rotational core) is never attached on the wall. There is no 


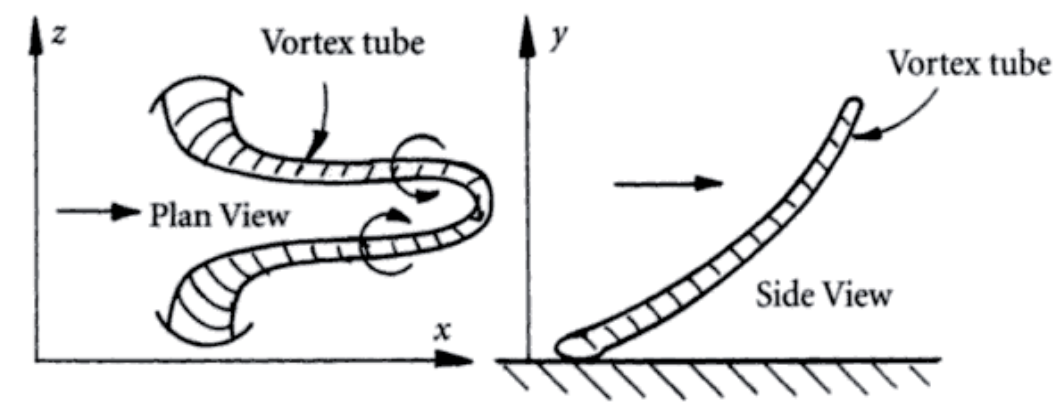

Figure 4. Vortex tube must break down to violate vorticity flux conservation law (copied from Davidson's book, Turbulence, published by Oxford University Press, 2004, see [7])

mechanism to support the switch from attachment to detachment, which would directly violate the vorticity flux conservation law and make the vorticity tube end inside the flow field. Actually, $\Lambda$ - vortex, not vorticity tube, is never attached on the wall (Figure 5).

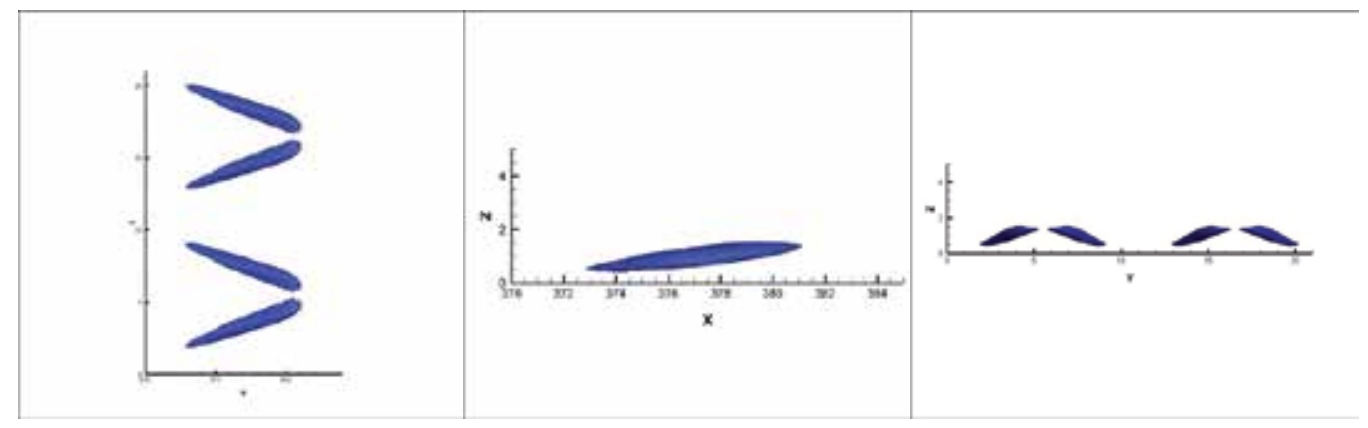

Figure 5. Projection of $\Lambda$-vortex on $x-y$ plane; $x-z$ plane; $y-z$ plane at $t=3.07 T$

7. Vortex ring auto-generation: Some literatures suggest that the multiple vortex rings in a vortex package are auto-generated. We must be very careful when using the term "autogeneration" since everything must be generated under certain mechanism and, in general, cannot be auto-generated in this world. Liu et al. [23] believe all vortex rings are generated by the shear layer instability. There is no exception. There is a vortex rollup (Figure 6), which forms a low speed zone in the middle of the $\Lambda$-vortex (Figure 7). The low-speed zone will form a strong shear and the shear layer will further develop vortex rings through Kelvin-Helmholtz-type instability (Figure 8.)

8. Turbulence bursting and intermittence: Bursting means sudden appearance of physical quantity fluctuation and sudden increases of friction and Reynolds stress. These were explained as some unstable modes' sudden growth and breakdown. 


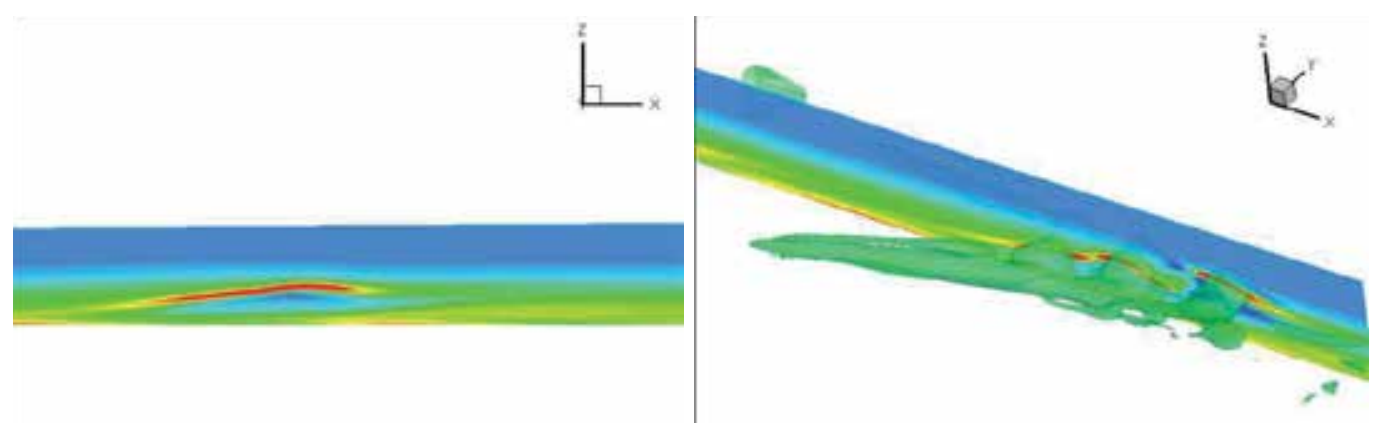

Figure 6. Vorticity rollup and vortex ring formation

$\begin{array}{llll}\text { Fourth ring } & \text { Third ring } & \text { Second ring } & \text { First ring }\end{array}$

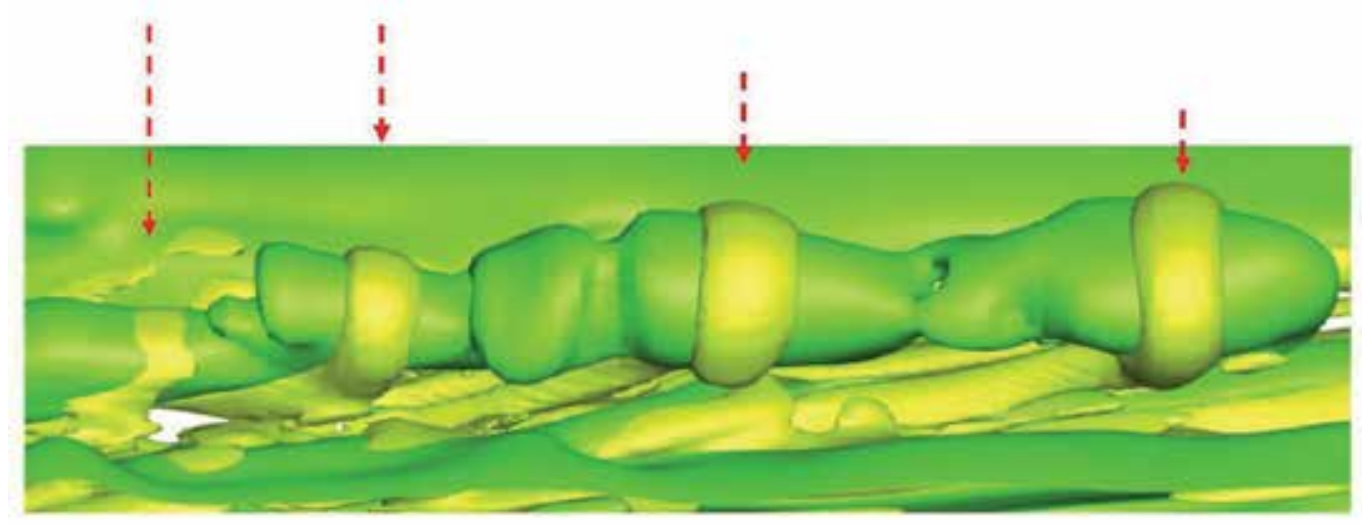

5025045065085105125145165185205225245265285

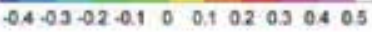

Figure 7. Low speed zone and vortex ring formation

However, that is not the case. Actually, this is caused by small length scale generation and fast motion of vortex rings, which has low speed in the ring center and low pressure in the rotation core center. The motion of vortex rings and vortex packages will cause the fluctuation. Turbulence cannot suddenly appear (bursting) and suddenly disappear (intermittence). Note that the vortex rings are moving with a selfrotation speed of around 10,000 circles per second. Even for a steady flow of such a vortex ring motion, it will cause a misunderstanding that the flow is strongly fluctuated in time if we installed a fixed probe inside the flow field. Turbulence bursting and intermittence are two important concepts in classical turbulence theory [33] but, unfortunately, they are basically misunderstandings. Turbulence cannot suddenly 


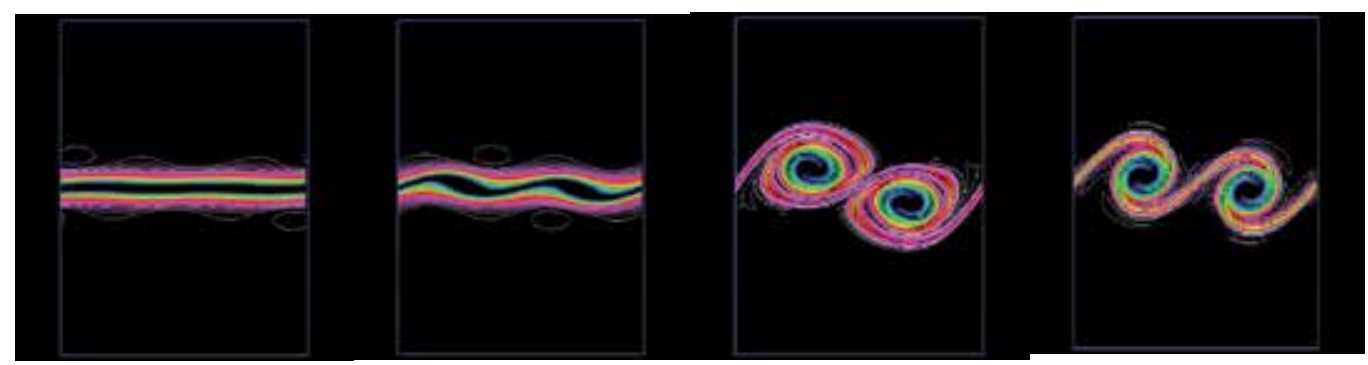

Figure 8. Kevin-Helmholtz instability

appear (bursting) and then suddenly disappear (intermittency). If turbulence can, there must be some superpower to manipulate the flow, which is impossible. These concepts are mistakenly formed by detection of flow fluctuations through fixed probes. Since the fast-rotating vortex rings have strong velocity gradient with low speed zones in the ring center and low pressure in the rotational core centers, the vortex package motion will show strong velocity and pressure fluctuations when the vortex package passes through these probes. Therefore, the velocity and pressure fluctuation, Reynolds stress, and surface friction will be quickly increased. After the vortex package left, the flow will recover and be quiet again. People usually call these "turbulence bursting and intermittency," which is really an incorrect understanding of the vortex package motion. The fluctuation including the frequencies and energy spectrum is determined by the vortex package structure and the vortex moving speed. Both "bursting" and "intermittency" can be reproduced by DNS for vortex package moving. Liu et al. [23] believe the turbulence generation cannot burst but is a very wellorganized flow activity with four steps: $\Lambda$-vortex root formation, vortex ring formation, sweeps, and small vortices generation. The intermittence is a misunderstanding of vortex package self-motion (rotation) and relative motion. Turbulence cannot suddenly appear (burst) and then suddenly disappear (intermittence). The "turbulence bursting" and "intermittence" must be studied deeply and current misunderstandings must be clarified. The turbulence bursting and intermittency can be reproduced by DNS through a fixed-position probe (Figure 9), which agrees very well with the experiment by Borodulin and Kachanov [1]. This clearly shows that the fluctuation is caused by uneven vortex package movement. It means that fluctuation (turbulence) is vortex package motion.

9. Richardson's [31] eddy cascade (Figure 10a) which was described by a poem: “Big whirls have little whirls that feed on their velocity, and little whirls have lesser whirls and so on to viscosity." This has been accepted by the turbulence community for a long time. However, no one was able to observe such a cascade. It is really suspicious as the experimental tool is so advanced today (the resolution of Cai's experiment is around 1 $\mu m$ ) but we still cannot observe such a cascade. We have to be suspicious that there is no such a cascade. Actually, we see the large vortex induce smaller vortices through sweep in our DNS (Figure 10b). 


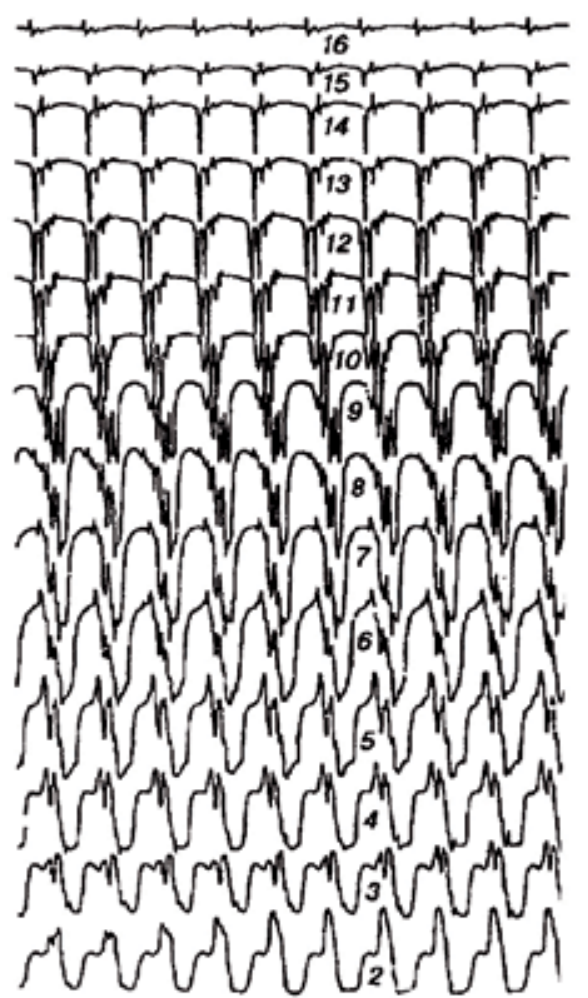

(a)

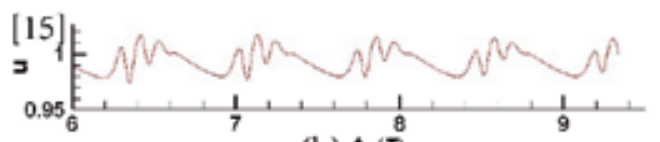

(b) $\mathbf{t}(\pi)$
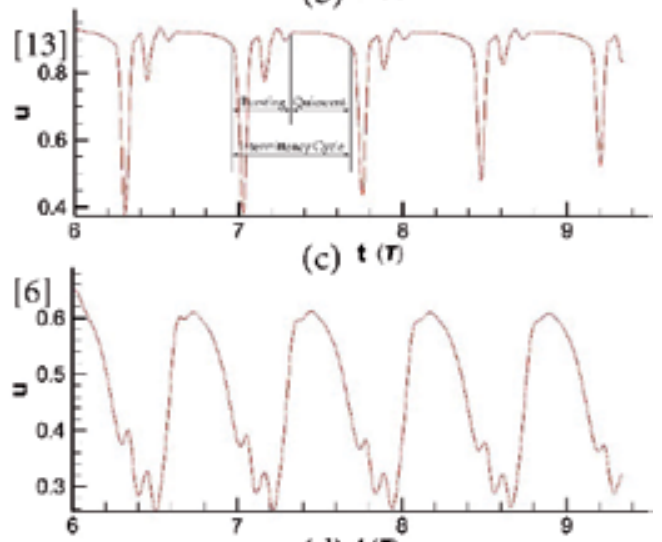

(d) $\mathbf{t}(n$

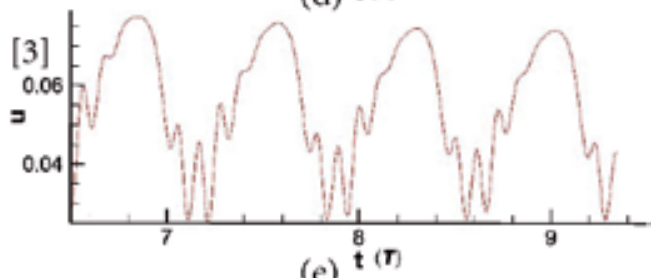

(b)

Figure 9. Comparison of experiment with DNS for turbulence intermittency

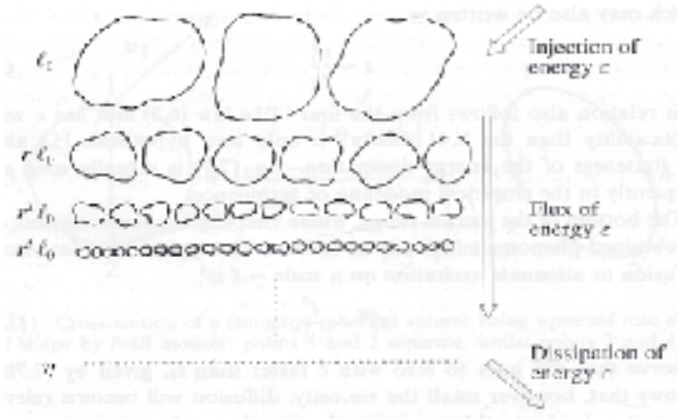

(a)

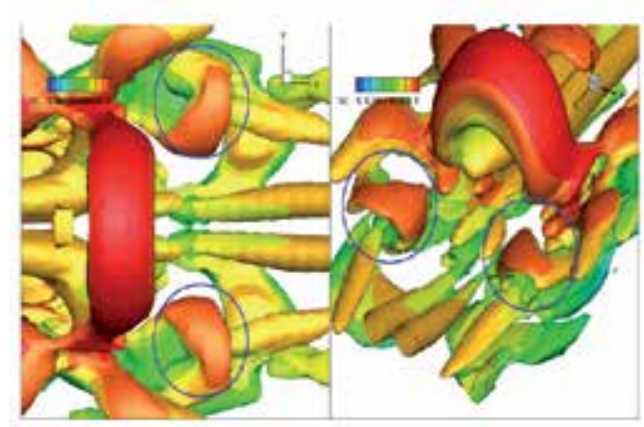

(b)

Figure 10. Richardson's eddy cascade (1928) and DNS observation on smaller vortices 
10. Energy transfer route: Kolmogorov [11] believed that the large eddy passes energy to smaller vortices through "vortex breakdown" with very short eddy turnover time, which is $l / u$ (see [7]), but no one was able to detect such a breakdown. Liu et al. [23] believe the large vortex passes the high energy through sweeps to generate positive spikes and further small vortices. Cai's experiment shows that when many small vortices appear, the large vortices are still alive and do not die as mothers. There is no "vortex breakdown" in any sense.

11. Bypass transition and free stream turbulence: If turbulence is generated by linear mode growth, nonlinear interaction, resonance, and vortex breakdown, current transition and turbulence theories have no ways to explain why we have "bypass transition" and "free stream turbulence." Liu et al. [23] believe flow transition is caused by the inherent property of fluids, that flow cannot tolerate shear and shear must transfer to rotation. In other words, laminar flow (shear-dominant) must transfer to turbulent flow (rotation-dominant) since the turbulent (rotation-dominant) state is a stable state. There is no necessary condition to have receptivity and linear growth for flow transition, and even no necessity to have $\Lambda$-vortex either.

These are just some sample questions to show that the classical or current turbulence theories are not able to give any convincing answer. Liu et al. believe one counterexample is good enough to overthrow a theory and we really do not need a second example. The current transition and turbulence theories have too many self-contradictions, definitely more than one or two.

Wallace [35] pointed out in his review paper: “... there has been remarkable progress in turbulent boundary layer research in the past 50 years, particularly in understanding the structural organization of the flow. Consensus exists that vortices drive momentum transport but not about the exact form of the vortices or how they are created and sustained." The authors have conducted a new high-order DNS with large number of grids to study the "turbulence generation and sustenance" and give exact form of the vortices or how they are created and sustained.

Many people misunderstand vorticity as rotation, vortex as vortex tube, and vorticity line as vortex line. Actually, as addressed above, vortex is a rotating core which consists of vortex lines with leaking, but vortex tube is a tube with vorticity lines without leakage, according to the definition in Davidson's book. Therefore, vorticity does not mean rotation, vortex is not vortex tube, and vorticity line is not vortex line. On the other hand, vortex line is part of vorticity lines and rotating vorticity is part of vorticity.

\section{Liu's new findings}

After 25 years of effort made by Liu and his students, the following new findings by highorder DNS were made:

- Mechanism of spanwise vorticity rollup 
- Mechanism of transfer from flow shear to rotation

- Mechanism of spanwise vortex formation and role of the linear unstable modes

- Mechanism of vortex root formation

- Mechanism of first ring-like vortex formation

- Mechanism of multiple vortex ring formation

- Mechanism of second sweep formation

- Mechanism of low-speed zone and high shear layer formation

- Mechanism of positive spike formation

- Mechanism of secondary and tertiary vortex formation

- Mechanism of U-shaped vortex formation

- Mechanism of small length vortices generation

- Mechanism of multiple-level high shear layer formation

- Mechanism of energy transfer paths from the large length scales to the small ones

- Mechanism of symmetry loss and flow chaos

- Mechanism of thickening of turbulence boundary layer

- Mechanism of high surface friction of turbulent flow

\section{Highlights of our new observations}

4.1 People in general accept that turbulence is generated by some unstable modes that experience linear growth, nonlinear interaction or resonance, and vortex breakdown to turbulence. However, it is not the case. Linear modes can grow and trigger the flow transition, but flow transition is caused by the inherent property of fluid that "shear must transform to rotation."

4.2 Rotational and non-rotational vorticity and Helmholtz velocity decomposition revisited Vorticity does not mean rotation as many people are confused by the two concepts. Actually, fluid particle motion can only be decomposed as symmetric tenser and non-symmetric tenser. The latter represents vorticity but not rotation. The vorticity can be further decomposed to rotational vorticity and non-rotational vorticity, which is different from Helmholtz decomposition of fluid particle motion. 


$$
\begin{aligned}
& \nabla \times \vec{V}=\Omega \vec{R}+(\nabla \times \vec{V}-\Omega \vec{R}) \approx \Omega \nabla \times \vec{V}+(1-\Omega) \nabla \times \vec{V} \\
& \text { In general } \vec{R} \neq \nabla \times \vec{V}
\end{aligned}
$$

4.3 Although vorticity must keep conservation, once vorticity rolls up from the wall, the shear must transfer to rotation (non-rotational vorticity becomes rotational vorticity and deformation disappears) inside the flow field. On the other hand, laminar flow, which is dominated by shear and instability, must transfer to turbulent flow, which is dominated by rotation and stability. When the fluid particle becomes rotation like a rigid body, there is no deformation and thus no dissipation, which is the least energy-consuming state.

4.4 The nature of flow transition is that fluids inside the flow field cannot tolerate the shear, and shear must transfer to rotation when the Reynolds number is large enough. Flow transition is the inherent property of fluid flow.

4.5 Although the linearly unstable modes are important for flow transition, they are small, cannot form vortex, and cannot cause flow transition directly through either absolute instability or convective instability. They can only stimulate vorticity "rollup," which could cause flow transition. All linear unstable modes play a same role to push the vorticity up from the wall (rollup.) The flow trend to change shear to rotation will occur inside the flow field.

4.6 Linear mode suppression has been developed for several decades with little success. Now we understand that these efforts are unsuccessful since any factor that can cause vorticity rollup, like gust, dust, noise, mosquito, fly, can lead to failure of unstable mode suppression. The key issue is to avoid vorticity "rollup" and shear layer formation.

4.7 Because the linear unstable modes are small and cannot form vortex (negligible), mainly the vorticity which made turbulence structure is originated from the original wall boundary Blasius solution with absolute vorticity increment by stretching and tilting. Vorticity does not mean rotation and should be further decomposed to rotational vorticity and non-rotational vorticity.

4.8 The analytic linear solution becomes discrepant from DNS at the very beginning. They do not agree with each other even in the very early stages.

4.9 Vortex and hairpin vortex

The theory that $\Lambda$-vortex self-deforms to hairpin vortex, as given by Hamma [8] and Moin et al. [30] has been accepted by the turbulence community for many years; however, it is unfounded since deformation is a motion and any motion must be driven by force and cannot self-deform. Considering $\Lambda$-vortex as a vortex tube without vorticity line leakage is a serious mistake in turbulence study. The $\Lambda$-vortex root and ring head are formed by totally different mechanisms and vortex ring is not part of $\Lambda$-vortex (Figure 11.) A low-speed zone is formed above the lambda-vortex to form a high shear due to the vortex root rotation. The first vortex ring is generated by the high shear layer ( $\mathrm{K}-\mathrm{H}$ type) instability near the tip of the $\Lambda$-structure. Multiple vortex rings are all formed by shear layer instability, which is generated by momentum deficit (Figure 12). 


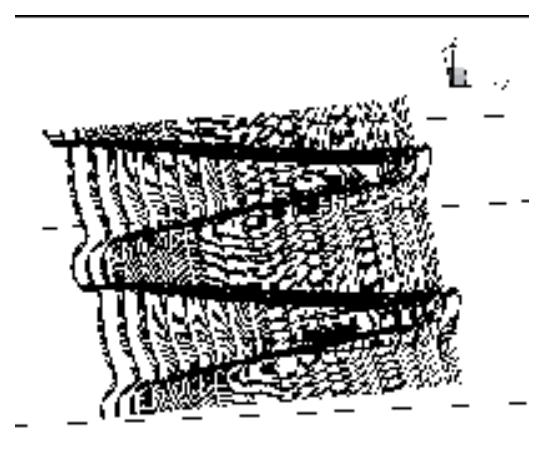

(a) Vorticity

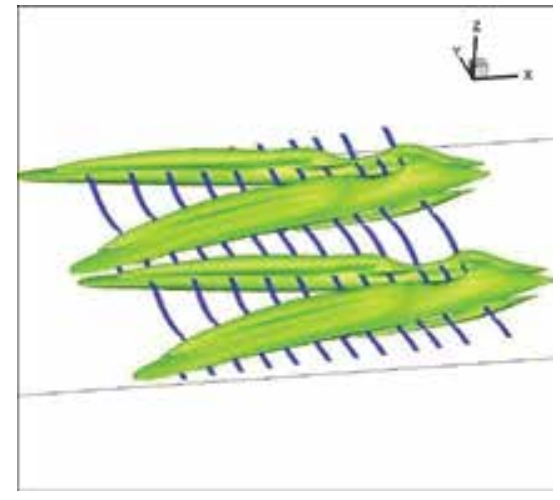

(b) Vortex

Figure 11. Vorticity and vortex of $\Lambda$-structure

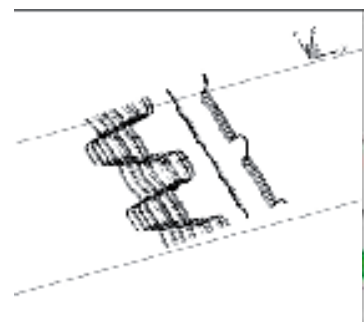

(a) Vorticity

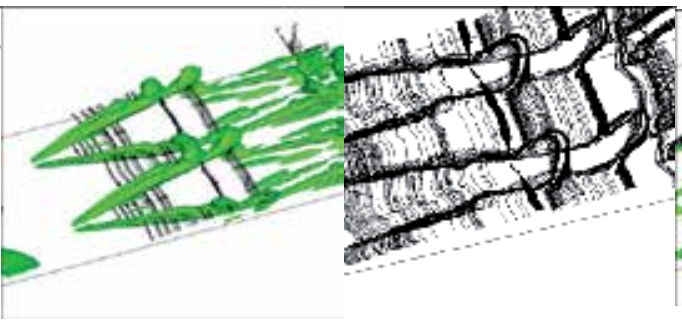

(b) Vortices

(c) Vorticity

(d) Vortices

Figure 12. Vorticity and vortices for multiple vortex rings

\subsection{Vortex breakdown}

"Vortex breakdown" is caused by faked visualization by using improper lambda2 values or Q-iso-surface. We can make various "vortex breakdowns" by using the same DNS data set with different lambda2 values (Figure 13). However, the vortex structures are very stable when they travel.

\subsection{Energy transfer path from larger vortex to smaller vortices}

The small vortices still need energy to survive, although rotation is the most stable state due to the minimized deformation and dissipation. In fact, the high energy is brought down to the lower boundary layer through fast large vortex rotation by multiple-level sweeps. Without these sweeps, all small vortices (turbulence) would dissipate quickly. Large vortex passed high energy through strong rotation (sweeps in particular) but definitely not through "vortex breakdown." 


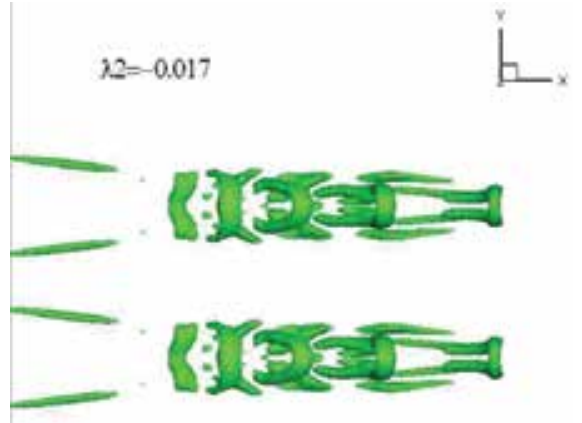

(a) Fake vortex breakdown (lambda2 $=-0.0017)$.

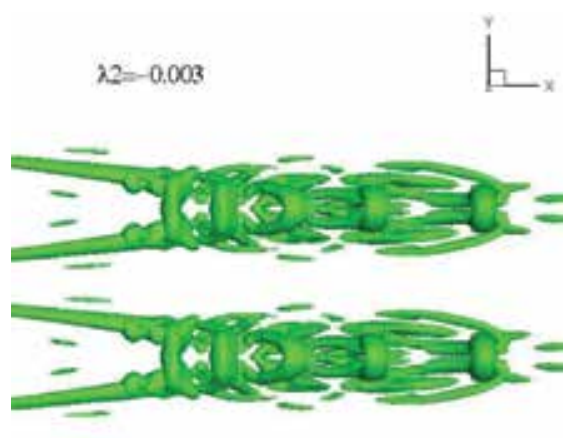

(b) No breakdown (lambda $=-0.003)$

Figure 13. Fake "vortex breakdown" made by choosing inappropriate lambda2 values

4.12 Highlights of our new observations - chaos

The loss of flow symmetry begins from the middle of the multiple-level vortex structure, which shows it is an inherent instability of the multiple-level vortex structure. There is no proof that the chaos is caused by strong environmental disturbances and/or nonperiodic spanwise boundary conditions.
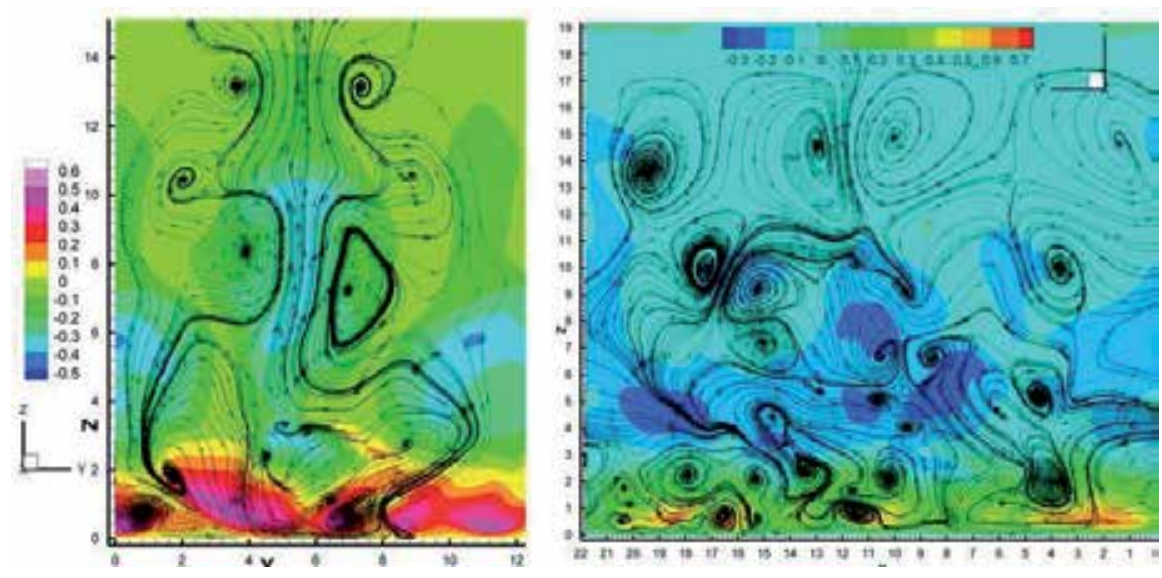

Figure 14. Asymmetry starts in the middle of the multi-level vortex package

\section{Some conceptual mistakes in fundamental fluid dynamics}

The authors believe some people have made at least several conceptual misunderstandings:

1. Considering vortex as "vortex tube": This is a major source of confusion. 
2. Considering vorticity as rotation: They are two different concepts.

3. Considering hairpin vortex is self-deformed from Lambda vortex: There is no selfdeformation.

4. Considering multiple vortex rings are auto-generated: Anything must be generated by certain mechanisms.

5. Considering vortex was first attached on the wall and then detached from the wall: Our textbooks say vortex legs can only lie down on the wall, but, in fact, vortex never attaches on the wall.

6. Considering small vortices are generated by large vortex breakdown: This is hypothesized by Richardson and Kolmokorov with no proof. The experiment conducted by Cai shows the large vortex is still there and even does not deform when a large number of small vortices are generated.

7. Considering vortex breaks down and then reconnects: Vortex ring and legs are never linked and they are separated as two different parts.

8. Considering turbulence is generated by unstable modes' linear growth, interaction, resonance, and breakdown either by absolute instability or convective instability: Linear modes can only trigger the vorticity rollup, but not part of the transition or part of the vortex structure.

9. Misunderstanding the uneven vortex package structure and package motion as "bursting" and "intermittency": This is caused by observation of fluid particle motion in a fixedframe Euler system. Turbulence looks like velocity and pressure fluctuation, but fluctuation is really caused uneven vortex package motion.

10. Not realizing the vortex ring has a very fast-rotating core (e.g., around 10,000 circles/ second) with large gradient in velocity and pressure.

11. Considering turbulent flow is a random motion: Turbulent flow cannot be random since fluid motion must follow conservation of mass, momentum, and energy.

Of course, these misunderstandings are hard to avoid as our pioneering scientists living in the 19th and early 20th centuries had neither computers nor high-resolution experimental instruments. They mainly presented hypotheses and assumptions, which must be reexamined. However, it would be an unpardonable mistake if we accept these hypotheses and teach our kids with the wrong concepts generation by generation without careful analyses by DNS and experiments.

\section{Liu's new theory on flow transition and turbulence generation}

By using high-order DNS in LBLT, Liu has revealed many new mechanisms, some of which are directly against the classical theory: 


\subsection{Nature of turbulence generation:}

1. Fluids cannot tolerate high shear and shear must transfer to rotation and form a very fast rotation core (Dr. Cai has given his experimental observation)

2. Turbulence is not generated by "vortex breakdown" but "vortex buildup"

3. "Shear layer instability" is the "mother of turbulence"

4. Turbulence small scales are generated by multiple level shear layers which are generated by multiple level sweeps, ejections, negative and positive spikes.

5. Large vortices provide energy to smaller vortices through fast rotation which causes strong sweeps.

\subsection{Nature of the flow transition}

1. Flow transition is mainly caused by vorticity rollup from the wall.

2. Flow transition is a vorticity redistribution and increment from near wall to whole boundary layer.

3. Flow transition is a process of non-rotational vorticity transferring to rotational vorticity. Actually, laminar flow dominated by shear (non-rotational vorticity) is an unstable state but turbulent flow dominated by rotational vorticity is a stable state (rigid rotating vortex ring will have no deformation and then no energy dissipation). Of course, there are still shear layers between vortex rings and energy is still needed to keep these small vortex rings alive. The energy is provided by large vortex through rotation, sweeps in particular.

\section{Conclusion}

The classical and current turbulence theories are filled with many self-contradictions and, therefore, must be revisited. DNS and high-resolution experiment will pave ways to provide correct concepts and theories for turbulence study. Turbulence consists of rotations with different sizes of vortices. Laminar flow is dominated by shear which is unstable and turbulence is dominated by rotation which is stable. The rigid rotation of fluids has least energy consumption since it has no deformation (energy dissipation).

Flow transition is not a process of "vortex breakdown" but "vortex buildup." Vorticiy has rotational and non-rotational parts. Flow transition is a process of transformation of nonrotational vorticity to rotational vorticity, while the shear is gradually reduced but rotation is strengthened.

Flow transition is a fluid inherent property that shear must transfer to rotation when Reynolds number is large, i.e., transfer to a stable state.

The role of unstable modes is to stimulate the vorticity rollup from the wall. All high shear layers are produced by the vortex structure with multiple-level vortex rings, multiple-level 
sweeps and ejections, and multiple-level positive and negative spikes near the laminar sublayers. The vortex rotation generates low-speed zones which cause the high shear.

“Vortex breakdown" never happens. "Turbulence" is not generated by "vortex breakdown" but by positive spikes and consequent high shear layers.

There is a universal mechanism for turbulence generation and sustenance - the energy is brought by large vortex structure through fast rotation with multiple-level sweeps.

Flow disordering is caused by the instability of multiple-level vortex packages.

\section{Acknowledgements}

Liu's new turbulence theory was presented by Chaoqun Liu, with help received from many of his students. The authors are grateful to participants including Zhining Liu, Hua Shan, Li Jiang, Lin Chen, Xiaobin Liu, Ping Lu, Yonghua Yan, and Yiqian Wang.

This work was originally supported by AFOSR Grant FA9550-08-1-0201 (United States), supervised by Dr. John Schmisseur and the Department of Mathematics at University of Texas at Arlington. The authors are grateful to Texas Advanced Computing Center (TACC) for providing computation hours. This work is accomplished by using Code DNSUTA, and was released by Dr. Chaoqun Liu at University of Texas at Arlington in 2009.

\section{Author details}

Chaoqun Liu* and Shuhyi Chen

*Address all correspondence to: cliu@uta.edu

University of Texas at Arlington, Arlington, TX, USA

\section{References}

[1] Borodulin, V.I., Kachanov, Y.S. Formation and development of coherent structures in a transitional boundary layer. J Appl Mech Tech Phys, 1995;36(4): 532-564.

[2] Chen, L., Liu, X., Oliveira, M., Tang, D., Liu, C. Vortical structure, sweep and ejection events in transitional boundary layer, Sci China, Series G, Physics, Mechanics, Astronomy, 2009;39(10): 1520-1526. 
[3] Chen, L., Liu, X., Oliveira, M., Liu, C. DNS for ring-like vortices formation and roles in positive spikes formation, AIAA Paper 2010-1471, Orlando, FL, USA, January 2010a.

[4] Chen, L., Tang, D., Lu, P., Liu, C. Evolution of the vortex structures and turbulent spots at the late-stage of transitional boundary layers, Sci China, Physics, Mechanics, Astronomy, January 2010b;53(1): 1-14.

[5] Chen, L., Liu, C. Numerical study on mechanisms of second sweep and positive spikes in transitional flow on a flat plate, J Comp Fluids, 2011a;40:28-41.

[6] Chen, L., Liu, X., Tang, D., Liu, C. Evolution of the vortex structures and turbulent spots at the late-stage of transitional boundary layers. Sci China, Physics, Mechanics Astronomy, 2011b;54(5): 986-990.

[7] Davidson, P.A., Turbulence: An Introduction for Scientists and Engineers. Page 123, Oxford University Press. ISBN 9780198529491, 2004.

[8] Hama, F.R., Nutant, J. Detailed flow-field observations in the transition process in a thick boundary layer. Proc. 1963 Heat Transfer \& Fluid Mech. Inst., Palo Alto, CA: Stanford Univ. Press, 1963; pp. 77-93.

[9] Jeong J., Hussain F. On the identification of a vortex, J Fluid Mech 1995, 285:69-94.

[10] Jiang, L., Chang, C. L. (NASA), Choudhari, M. (NASA), Liu, C. Cross-Validation of DNS and PSE Results for Instability-Wave Propagation, AIAA Paper 2003-3555, The 16th AIAA Computational Fluid Dynamics Conference, Orlando, Florida, June 23-26, 2003.

[11] Kolmogorov, A.N. (1941). The local structure of turbulence in incompressible viscous fluid for very large Reynolds numbers. Proc Royal Soc Lond, Series A: Math Phys Sci 1991;434: 9-13. Bibcode 1991RSPSA.434....9K. doi:10.1098/rspa.1991.0075.

[12] Liu, C., Liu, Z. Multigrid mapping and box relaxation for simulation of the whole process of flow transition in 3-D boundary layers. J Comput Phys, 1995;119: 325-341.

[13] Liu, Z., Xiong, G., Liu, C., Direct numerical simulation for the whole process of transition on 3-D airfoils. AIAA paper, 1996; AIAA 96-2081.

[14] Liu, C., Liu, Z. Direct Numerical Simulation for Flow Transition Around Airfoils, Proceedings of First AFOSR International Conference on DNS/LES, Louisiana Tech University, Ruston, Louisiana, August 4-8, 1997.

[15] Liu, C., Chen, L. Study of mechanism of ring-like vortex formation in late flow transition, AIAA Paper 2010-1456, Orlando, FL, 2010a.

[16] Liu, X., Chen, L., Oliveira, M., Tang, D., Liu, C. DNS for late stage structure of flow transition on a flat-plate boundary layer, AIAA Paper 2010-1470, Orlando, FL, January $2010 b$. 
[17] Liu, C., Chen, L. Study of mechanism of ring-like vortex formation in late flow transition, AIAA Paper 2010-1456, Orlando, FL, January 2010c.

[18] Liu, X., Chen, Z., Liu, C. Late-stage vortical structures and eddy motions in transitional boundary layer status, Chinese Phys Lett, 2010d;27(2): 024706-1-4.

[19] Liu, C., Chen, L., Lu, P. New findings by high order DNS for late flow transition in a boundary layer. J Model Simul Engin, 2011a; 2011(721487): 1-16.

[20] Liu, C., Chen, L. Parallel DNS for vortex structure of late stages of flow transition. J Comput Fluids, 2011b;45: 129-137.

[21] Liu, C. Numerical and theoretical study on "Vortex Breakdown," Int J Comput Math, 2011c;88(17): 3702-3708.

[22] Liu, C., Chen, L., Lu, P., Liu, X. Study on multiple ring-like vortex formation and small vortex generation in late flow transition on a flat plate, theoretical and numerical fluid dynamics. 2013; 27(1): 41-70.

[23] Liu, C., Yan, Y., Lu, P. Physics of turbulence generation and sustenance in a boundary layer, Comput Fluids, 2014;102 (2014): 353-384.

[24] Lu, P., Liu, C. Numerical study of mechanism of u-shaped vortex formation, AIAA Paper 2011-0286, Orlando, Florida, USA, January 4-7, 2011.

[25] Lu, P., Wang, Z., Chen, L., Liu, C. Numerical study on U-shaped vortex formation in late boundary layer transition. Comput Fluids. 2011a;55: 36-47.

[26] Lu, P., Liu, C. Numerical study on mechanism of small vortex generation in boundary layer transition. AIAA Paper 2011-0287, 2011b, Orlando, Florida, USA, January 4-7, 2011.

[27] Lu, P., Liu, C. DNS study on mechanism of small length scale generation in late boundary layer transition. Physica D: Nonlinear Phen, 2011c;241 (2012): 11-24.

[28] Lu, P., Thampa, M, Liu, C. Numerical Study on Randomization in Late Boundary Layer Transition, AIAA 2012-0748, 2012, Nashville, TN, January 2012.

[29] Marshak, A. 3D Radiative Transfer in Cloudy Atmospheres. Springer. ISBN 9783540239581, 2005; p. 76.

[30] Moin, P., Leonard, A. Kim, J. Evolution of curved vortex filament into a vortex ring. Phys Fluids, 29(4): 955-963.

[31] Richardson, L. F., Weather Prediction by Numerical Process (Cambridge Univ. Press, 1922).

[32] Rist, U., et al. Turbulence mechanism in Klebanoff transition: a quantitative comparison of experiment and direct numerical simulation. J Fluid Mech, 2002; 459:217-243. 
[33] Schlichting, H., Gersten, K. Boundary Layer Theory, Springer, 8th revised edition, 2000, ISBN 3-540-66270-7.

[34] USA Today, Turbulence theory gets a bit choppy, September 10, 2006. http:// www.usatoday.com/tech/science/columnist/vergano/2006-09-10-turbulence_x.htm.

[35] Wallace, J.M. Highlights from 50 years of turbulent boundary layer research. J Turbulence, 2013;13(53):1-70.

[36] Wu, X., Moin, P. Direct numerical simulation of turbulence in a nominally zero-pressure gradient flat-plate boundary layer, JFM, 630: 5-41, 2009.

[37] Yan, Y., Liu, C. Shear Layer Stability Analysis in Later Boundary Layer Transition and MVG controlled flow, AIAA2013- 0531, January 6-10, 2013.

[38] Yan, Y., Chen, C., Fu, H., Liu, C. DNS study on $\lambda$-vortex and vortex ring formation in flow transition at Mach number 0.5. J Turbulence, 2014;5(1): 1-21. 
Chapter 2

\title{
Modeling of Wave Propagation from Arbitrary Depths to Shallow Waters - A Review
}

\author{
José Simão Antunes do Carmo \\ Additional information is available at the end of the chapter \\ http://dx.doi.org/10.5772/61866
}

\begin{abstract}
Numerical models are a useful instrument for studying complex superposition of wave-wave and wave-current interactions in coastal and estuarine regions and to investigate the interaction of waves with complex bathymetries or structures built in nearshore areas. Moreover, since their applications are significantly less expensive and more flexible than the construction of physical models, they are a convenient tool to support design. The ability of the standard Boussinesq and Serre or Green and Naghdi equations to reproduce these nonlinear processes is well known. However, these models are restricted to shallow water conditions, and addition of other terms of dispersive origin has been considered since the 1990s, particularly for approximations of the Boussinesq-type. To allow applications in a greater range of $h_{0} / \lambda$, other than shallow waters, where $h_{0}$ is the water depth at rest and $\lambda$ is the wavelength, a new set of Serre-type equations, with additional terms of dispersive origin, is developed and tested with the available data and with a numerical solution of a Boussinesq-type model, also improved with dispersive characteristics. Explicit and implicit methods of finite-difference are implemented to solve both approximations of Boussinesq and Serre types with improved dispersive characteristics. A finite element method is also implemented to solve an extension of a Boussinesq-type model that takes into account wave-current interactions. Application examples to solve real-world problems are shown and discussed. The performances of both 1HD models are compared with experimental data of very demanding applications, namely: (i) a highly nonlinear solitary wave propagating up a slope and reflecting from a vertical wall and (ii) a periodic wave propagating in intermediatedepth waters upstream a trapezoidal bar, followed by very shallow waters over the bar, and again in intermediate-depth water conditions downstream.
\end{abstract}

Keywords: Extended Boussinesq equations, Extended Serre equations, Dispersive characteristics, Intermediate waters, Numerical methods 


\section{Introduction}

In recent decades, significant advances have been made in developing mathematical and numerical models to describe the entire phenomena observed in shallow water conditions. Indeed, not only our understanding of the phenomena has significantly improved but also the computational capabilities that are available have also increased considerably. In this context, we can now use more powerful and more reliable tools in the design of structures commonly used in coastal environments.

By the end of the 1970s, due to the lack of sufficiently deep knowledge, but above all for lack of computing power, the use of the linear wave theory for the simulation of phenomena, such as refraction and diffraction of waves, was common practice. In the 1980s, other models that take into account not only the refraction but also the diffraction process have been proposed and commonly used by [12] Berkhoff et al., 1982, [21] Kirby and Dalrymple, 1983, [13] Booij, 1983, [20] Kirby, 1984, and [15] Dalrymple, 1988, among many others. However, as they are based on the linear theory, those models should not be utilized in shallow water conditions.

As noted in [6], even at that time models based on the Saint-Venant equations (see also [32] Saint-Venant, 1871) "were frequently used in practical applications. However, as has been widely demonstrated, in shallow water conditions and for some types of waves, models based on a non-dispersive theory, of which the Saint-Venant model is an example, are limited and are not usually able to compute satisfactory results over long periods of analysis" (see also [33]). Nowadays, it is generally accepted that for practical applications the combined gravity wave effects in shallow water conditions must be taken into account. In addition, the refraction and diffraction processes, the swelling, reflection and breaking waves, all have to be considered.

Also according to [6] "a number of factors have made it possible to employ increasingly complex mathematical models". Indeed, not only has there been great improvement in our theoretical knowledge of the phenomena involved but also the numerical methods have been used more efficiently. The great advances made in computer technology, especially since the 1980s, improving information processing and enabling large amounts of data to be stored, have made possible the use of more mathematical models, of greater complexity and with fewer restrictions. Indeed, only models of order $\sigma^{2}\left(\sigma=h_{0} / \lambda\right.$, where $h_{0}$ and $\lambda$ represent, respectively, depth and wavelength characteristics) or greater, of the Boussinesq or Serre types [14, 35], are able to describe the entire dispersive and nonlinear interaction process of generation, propagation and run-up of waves resulting from wave-wave and wave-current interactions. It is also worth to point out that in more complex problems, such as wave generation by seafloor movement, propagation over uneven bottoms, and added breaking effects, high-frequency waves can arise as a consequence of nonlinear interaction.

In the past few years, the possibility of using more powerful computational facilities, and the technological evolution and sophistication of control systems have required thorough theoretical and experimental research designed to improve the knowledge of coastal hydrodynamics. Numerical methods aimed for the applications in engineering fields that are more sophisticated and with a higher degree of complexity have also been developed. 
In Section 2, the general shallow water wave theory is used to develop different mathematical approaches, which are nowadays the basis of the most sophisticated models in hydrodynamics and sedimentary dynamics. Extensions of the equations for intermediate water of the more general approaches (order $\sigma^{2}$ ) are presented in Section 3. Numerical formulations of the models and appropriate application examples are presented in Section 4. Firstly, a submerged dissipation platform to protect the Bugio lighthouse, situated at the Tagus estuary mouth, Lisbon, is designed and tested numerically. The second application is a real-world problem concerning coastal protection, using a submerged structure to force the breaking waves offshore. The third example shows the agitations established in the port of Figueira da Foz, Portugal, for different sea states, with the objective of designing new protective jetties, or extend the existing ones. Finally, the fourth example shows the performance of the extended Serre equations for the propagation of a wave in very demanding conditions. Conclusions and future developments are given in Section 5 .

\section{Mathematical formulations}

We start from the fundamental equations of the Fluid Mechanics, written in Euler's variables, relating to a three-dimensional and quasi-irrotational flow of a perfect fluid [Euler equations, or Navier-Stokes equations with the assumptions of non-compressibility $(d \rho / d t=d i \vec{v}=0)$, irrotationality $(\operatorname{rot} \vec{v}=0)$ and perfect fluid (dynamic viscosity, $\mu=0)$ ]:

$$
\begin{aligned}
& u_{x}+v_{y}+w_{z}=0 \\
& u_{t}+u u_{x}+v u_{y}+w u_{z}=-p_{x} / \rho \\
& v_{t}+u v_{x}+v v_{y}+w v_{z}=-p_{y} / \rho \\
& w_{t}+u w_{x}+v w_{y}+w w_{z}=-p_{z} / \rho-g \\
& u_{z}=w_{x} ; v_{z}=w_{y} ; v_{x}=u_{y}
\end{aligned}
$$

with $p=0$ at $z=\eta(x, y, t), \quad w=\eta_{t}+u \eta_{x}+v \eta_{y}$ at $z=\eta(x, y, t)$, and $w=\xi_{t}+u \xi_{x}+v \xi_{y}$ at $z=-h_{0}+\xi(x, y, t)$. In these equations $\rho$ is density, $t$ is time, $g$ is gravitational acceleration, $p$ is pressure, $\eta$ is free surface elevation, $\xi$ is bottom, and $u, v, w$ are velocity components. Defining the dimensionless quantities $\varepsilon=a / h_{0}$ and $\sigma=h_{0} / \lambda$, in which $a$ is a characteristic wave amplitude, $h_{0}$ represents water depth, and $\lambda$ is a characteristic wavelength, we proceed with suitable nondimensional variables:

$$
\begin{aligned}
& x^{\prime}=x / \lambda, y^{\prime}=y / \lambda, z^{\prime}=z / h_{0}, \eta^{\prime}=\eta / a, \xi^{\prime}=\xi / h_{0}, t^{\prime}=t \sqrt{g h_{0}} / \lambda=t c_{0} / \lambda, p^{\prime}=p /\left(\rho g h_{0}\right), \\
& u^{\prime}=u /\left(a \sqrt{g / h_{0}}\right)=u h_{0} /\left(a c_{0}\right), v^{\prime}=v /\left(a \sqrt{g / h_{0}}\right)=v h_{0} /\left(a c_{0}\right), w^{\prime}=w \lambda /\left(a h_{0} \sqrt{g / h_{0}}\right)=w \lambda /\left(a c_{0}\right),
\end{aligned}
$$

where $c_{0}$ represents critical celerity, given by $c_{0}=\left(g h_{0}\right)^{1 / 2}$, and, as above, $\eta$ is free surface elevation, $\xi$ represents bathymetry, $u, v$ and $w$ are velocity components, and $p$ is pressure. In 
dimensionless variables, without the line over the variables, the fundamental equations and the boundary conditions are written [5]:

a. Fundamental equations

$$
\begin{array}{ll}
u_{x}+v_{y}+w_{z}=0 & \mathrm{a} \\
\varepsilon u_{t}+\varepsilon^{2} u u_{x}+\varepsilon^{2} v u_{y}+\varepsilon^{2} w u_{z}=-p_{x} & \mathrm{~b} \\
\varepsilon v_{t}+\varepsilon^{2} u v_{x}+\varepsilon^{2} v v_{y}+\varepsilon^{2} w v_{z}=-p_{y} & \mathrm{c} \\
\varepsilon \sigma^{2} w_{t}+\varepsilon^{2} \sigma^{2} u w_{x}+\varepsilon^{2} \sigma^{2} v w_{y}+\varepsilon^{2} \sigma^{2} w w_{z}=-p_{z}-1 & \mathrm{~d} \\
u_{z}=\sigma^{2} w_{x} ; v_{z}=\sigma^{2} w_{y} ; v_{x}=u_{y} & \mathrm{e}
\end{array}
$$

b. Boundary conditions

$$
\begin{array}{ll}
w=(1 / \varepsilon) \xi_{t}+u \xi_{x}+v \xi_{y}, z=-1+\xi & \mathrm{a} \\
w=\eta_{t}+\varepsilon u \eta_{x}+\varepsilon v \eta_{y}, z=\varepsilon \eta & \mathrm{b} \\
p=0, z=\varepsilon \eta & \mathrm{c}
\end{array}
$$

Integrating the first equation 2(a) between the bed $-1+\xi$ and the free surface $\varepsilon \eta$, taking into account 3(a) and 3(b), yields the continuity equation (4):

$$
[\eta-(1 / \varepsilon) \xi]_{t}+[(1-\xi+\varepsilon \eta) \bar{u}]_{x}+[(1-\xi+\varepsilon \eta) \bar{v}]_{y}=0
$$

where the bar over the variables represents the average value along the vertical. Then, accepting the fundamental hypothesis of the shallow water theory, $\sigma=h_{0} / \lambda<<1$, and developing the dependent variables in power series of the small parameter $\sigma^{2}$, that is

$$
f=\sum_{i=0}^{\infty}\left(\sigma^{2}\right)^{i} f_{i}, \text { for } f=(u, v, w, p, \eta, \xi, A)
$$

where $A=u_{x}+v_{y^{\prime}}$ from continuity 2(a), and with 3(a) and 3(b), the following equations are obtained:

$$
\begin{gathered}
w_{0}=-\left(z+1-\xi_{0}\right) A_{0}+w_{0}^{*} \\
w_{0}^{* *}=-\left(1+\varepsilon \eta_{0}-\xi_{0}\right) A_{0}+w_{0}^{*}
\end{gathered}
$$


where the simple and double asterisk represent the variables values at the bottom and at the surface, respectively. Of 2(e) we obtain, successively [34]:

$$
\begin{gathered}
u_{0}=u_{0}(x, y, t) \\
v_{0}=v_{0}(x, y, t) \\
u_{1}=-(1 / 2)\left(z+1-\xi_{0}\right)^{2} A_{0 x}+\left(z+1-\xi_{0}\right)\left(\xi_{0 x} A_{0}+w_{0 x}^{*}\right)+u_{1}^{*} \\
v_{1}=-(1 / 2)\left(z+1-\xi_{0}\right)^{2} A_{0 y}+\left(z+1-\xi_{0}\right)\left(\xi_{0 y} A_{0}+w_{0 y}^{*}\right)+v_{1}^{*}
\end{gathered}
$$

so that the average values of the horizontal components of the velocity, on the vertical, are given by:

$$
\begin{aligned}
\bar{u}=u_{0} & +\sigma^{2} u_{1}^{*}-\left(\sigma^{2} / 6\right)\left(1+\varepsilon \eta_{0}-\xi_{0}\right)^{2} A_{0 x} \\
& +\left(\sigma^{2} / 2\right)\left(1+\varepsilon \eta_{0}-\xi_{0}\right)\left(\xi_{0 x} A_{0}+w_{0 x}^{*}\right)+O\left(\sigma^{4}\right) \\
\bar{v}=v_{0} & +\sigma^{2} v_{1}^{*}-\left(\sigma^{2} / 6\right)\left(1+\varepsilon \eta_{0}-\xi_{0}\right)^{2} A_{0 y} \\
& +\left(\sigma^{2} / 2\right)\left(1+\varepsilon \eta_{0}-\xi_{0}\right)\left(\xi_{0 y} A_{0}+w_{0 y}^{*}\right)+O\left(\sigma^{4}\right)
\end{aligned}
$$

On the other hand, taking into account that,

$$
f=f_{0}+O\left(\sigma^{2}\right) \text { for } f=\left(u, v, \eta, \xi, w^{*}\right)
$$

from (5) and (9) we obtain:

$$
\begin{aligned}
u^{* *}= & \bar{u} \\
- & \left(\sigma^{2} / 3\right)(1+\varepsilon \eta-\xi)^{2} \bar{A}_{x} \\
& +\left(\sigma^{2} / 2\right)(1+\varepsilon \eta-\xi)\left(\xi_{x} \bar{A}+w_{x}^{*}\right)+O\left(\sigma^{4}\right) \\
v^{* *}= & \bar{v}-\left(\sigma^{2} / 3\right)(1+\varepsilon \eta-\xi)^{2} \bar{A}_{y} \\
& +\left(\sigma^{2} / 2\right)(1+\varepsilon \eta-\xi)\left(\xi_{y} \bar{A}+w_{y}^{*}\right)+O\left(\sigma^{4}\right)
\end{aligned}
$$

Representing by $\Gamma=w_{t}+\varepsilon u w_{x}+\varepsilon v w_{y}+\varepsilon w w_{z}$ the vertical acceleration of the particles, we get $\Gamma=w_{0 t}+\varepsilon u_{0} w_{0 x}+\varepsilon v_{0} w_{0 y}+\varepsilon w_{0} w_{0 z}+O\left(\sigma^{2}\right)$, and from (6), (7) and (11) the following approach is obtained:

$$
\begin{gathered}
\Gamma=-(z+1-\xi)\left[\bar{A}_{t}+\varepsilon \bar{u} \bar{A}_{x}+\varepsilon \bar{v} \bar{A}_{y}-\varepsilon \bar{A}^{2}\right] \\
+\left[w_{t}^{*}+\varepsilon \bar{u} w_{x}^{*}+\varepsilon \bar{v} w_{y}^{*}\right]+O\left(\sigma^{2}\right)
\end{gathered}
$$


in which the terms within the first parenthesis (straight parenthesis) represent the vertical acceleration when the bottom is horizontal, and the terms within the second parenthesis (straight parenthesis) represent the vertical acceleration along the real bottom. It should be noted that equation $2(\mathrm{~d})$ can be written as:

$$
\varepsilon \sigma^{2} \Gamma=-p_{z}-1
$$

where, by vertical integration between the bottom and the surface, the pressure $p$ on the surface is obtained as:

$$
\begin{aligned}
& p_{x}^{* *}=\varepsilon \eta_{x}\left(\varepsilon \sigma^{2} \Gamma^{* *}+1\right) \\
& p_{y}^{* *}=\varepsilon \eta_{y}\left(\varepsilon \sigma^{2} \Gamma^{* *}+1\right)
\end{aligned}
$$

which, along with 2(b) and 2(c), allow us to obtain [34]:

$$
\begin{aligned}
& \left(u_{t}+\varepsilon u u_{x}+\varepsilon v u_{y}+\varepsilon w u_{z}\right)^{* *}+\eta_{x}\left(1+\varepsilon \sigma^{2} \Gamma^{* *}\right)=0 \\
& \left(v_{t}+\varepsilon u v_{x}+\varepsilon v v_{y}+\varepsilon w v_{z}\right)^{* *}+\eta_{y}\left(1+\varepsilon \sigma^{2} \Gamma^{* *}\right)=0
\end{aligned}
$$

or even, given that $\left(f_{s}\right)^{* *}=f_{s}^{* *}-\varepsilon\left(f_{z}\right)^{* *} \eta_{s}$, where $f=(u, v)$ and $s=(x, y, t)$ :

$$
\begin{aligned}
& u_{t}^{* *}+\varepsilon u^{* *} u_{x}^{* *}+\varepsilon v^{* *} u_{y}^{* *}+\eta_{x}\left(1+\varepsilon \sigma^{2} \Gamma^{* *}\right)=0 \\
& v_{t}^{* *}+\varepsilon u^{* *} v_{x}^{* *}+\varepsilon v^{* *} v_{y}^{* *}+\eta_{y}\left(1+\varepsilon \sigma^{2} \Gamma^{* *}\right)=0
\end{aligned}
$$

By developing expressions (17) in second approach (order 2 in $\sigma^{2}$ ), the following equations of motion (18) are obtained (for details see [34]):

$$
\begin{aligned}
\bar{u}_{t} & +\varepsilon \bar{u}_{x}+\varepsilon \bar{v} \bar{u}_{y}+\eta_{x} \\
& +\sigma^{2}\left\{\left[(2 / 3)(\varepsilon \eta-\xi)_{x}+(1 / 2) \xi_{x}\right] P+(1 / 3)(1+\varepsilon \eta-\xi) P_{x}\right\} \\
& +\sigma^{2}\left[\varepsilon \eta_{x} Q+(1 / 2)(1+\varepsilon \eta-\xi) Q_{x}\right]+\sigma^{4}=0 \\
\bar{v}_{t} & +\varepsilon \bar{u}_{x}+\varepsilon \overline{v v}_{y}+\eta_{y} \\
& +\sigma^{2}\left\{\left[(2 / 3)(\varepsilon \eta-\xi)_{y}+(1 / 2) \xi_{y}\right] P+(1 / 3)(1+\varepsilon \eta-\xi) P_{y}\right\} \\
& +\sigma^{2}\left[\varepsilon \eta_{y} Q+(1 / 2)(1+\varepsilon \eta-\xi) Q_{y}\right]+\sigma^{4}=0 \\
P= & (1+\varepsilon \eta-\xi)\left(\varepsilon \bar{A}^{2}-\varepsilon \bar{u} \bar{A}_{x}-\varepsilon \bar{v} \bar{A}_{y}-\bar{A}_{t}\right) \\
Q & =w_{t}+\varepsilon \bar{u} w_{x}+\varepsilon \bar{v} w_{y} \\
w & =(1 / \varepsilon) \xi_{t}+\bar{u} \xi_{x}+\bar{v} \xi_{y} \\
\bar{A} & =\bar{u}_{x}+\bar{v}_{y}
\end{aligned}
$$


where, likewise, the bar over the variables represents the average value along the vertical. In dimensional variables and with a solid/fixed bottom $\left(\xi_{t}=0\right)$, the complete set of equations is written, in second approach:

$$
\begin{aligned}
h_{t} & +(h \bar{u})_{x}+(h \bar{v})_{y}=0 \\
\bar{u}_{t} & +\bar{u}_{x}+\bar{v}_{y}+g \eta_{x} \\
& +\left[(2 / 3) h_{x}+(1 / 2) \xi_{x}\right] P+(1 / 3) h P_{x}+h_{x} Q+(1 / 2) h Q_{x}=0 \\
\bar{v}_{t} & +\bar{u}_{x}+\overline{v v}_{y}+g \eta_{y} \\
& +\left[(2 / 3) h_{y}+(1 / 2) \xi_{y}\right] P+(1 / 3) h P_{y}+h_{y} Q+(1 / 2) h Q_{y}=0 \\
P & =h\left(\bar{A}^{2}-\bar{u} \bar{A}_{x}-\bar{v} \bar{A}_{y}-\bar{A}_{t}\right) \\
Q & =w_{t}+\bar{u} w_{x}+\bar{v} w_{y} \\
w & =\bar{u}_{x}+\bar{v} \xi_{y} \\
\bar{A} & =\bar{u}_{x}+\bar{v}_{y}
\end{aligned}
$$

where $h=h_{0}-\xi+\eta$ is total water depth. The one-dimensional form (1HD) of the equation system (19) is written, also with a fixed bottom:

$$
\begin{aligned}
& h_{t}+(\bar{u} h)_{x}=0 \\
& h \bar{u}_{t}+h \bar{u} \bar{u}_{x}+g h \eta_{x}+\left[h^{2}(P / 3+Q / 2)\right]_{x}+\xi_{x} h(P / 2+Q)=0 \\
& P=-h\left(\bar{u}_{x t}+\bar{u}_{x x}-\bar{u}_{x}^{2}\right) \\
& Q=\xi_{x}\left(\bar{u}_{t}+\bar{u} \bar{u}_{x}\right)+\xi_{x x} \bar{u}^{2}
\end{aligned}
$$

Assuming additionally a relative elevation of the surface due to the waves $\left(\varepsilon=a / h_{0}\right)$ having a value close to the square of the relative depth $\left(\sigma=h_{0} / \lambda\right)$, i.e. $O(\varepsilon)=O\left(\sigma^{2}\right)$, from the system of equations (18), and at the same order of approximation, the following approach is obtained, in dimensional variables:

$$
\begin{aligned}
& h_{t}+(h \bar{u})_{x}+(h \bar{v})_{y}=0 \\
& \bar{u}_{t}+\bar{u} \bar{u}_{x}+\bar{v} \bar{u}_{y}+g \eta_{x}-\left[(1 / 6) \xi_{x}\right] P+(1 / 3) h_{r} P_{x}+(1 / 2) h_{r} Q_{x}=0 \\
& \bar{v}_{t}+\bar{u}_{x}+\overline{v v}_{y}+g \eta_{y}-\left[(1 / 6) \xi_{y}\right] P+(1 / 3) h_{r} P_{y}+(1 / 2) h_{r} Q_{y}=0
\end{aligned}
$$

where $h_{r}=h_{0}-\xi$ is the water column height at rest, $P$ and $Q$ are given by $P=-\left(h_{0}-\xi\right)\left(\bar{u}_{x}+\bar{v}_{y}\right)_{t}$ and $Q=\left(\bar{u} \xi_{x}+\bar{v} \xi_{y}\right)_{t}$. The momentum equations are written as: 


$$
\begin{aligned}
\bar{u}_{t}+\bar{u} \bar{u}_{x} & +\bar{v}_{y}+g \eta_{x}+(1 / 6) h_{r} \xi_{x}\left(\bar{u}_{x}+\bar{v}_{y}\right)_{t}-(1 / 3) h_{r}^{2}\left(\bar{u}_{x}+\bar{v}_{y}\right)_{x t} \\
& +(1 / 3) h_{r} \xi_{x}\left(\bar{u}_{x}+\bar{v}_{y}\right)_{t}+(1 / 2) h_{r}\left(\bar{u} \xi_{x}+\bar{v} \xi_{y}\right)_{x t}=0 \\
\bar{v}_{t}+\overline{u v}_{x} & +\overline{v v}_{y}+g \eta_{y}+(1 / 6) h_{r} \xi_{y}\left(\bar{u}_{x}+\bar{v}_{y}\right)_{t}-(1 / 3) h_{r}^{2}\left(\bar{u}_{x}+\bar{v}_{y}\right)_{y t} \\
& +(1 / 3) h_{r} \xi_{y}\left(\bar{u}_{x}+\bar{v}_{y}\right)_{t}+(1 / 2) h_{r}\left(\bar{u} \xi_{x}+\bar{v} \xi_{y}\right)_{y t}=0
\end{aligned}
$$

with $\xi_{t}=0$, the complete system of equations (24) is obtained:

$$
\begin{aligned}
h_{t}+(h \bar{u})_{x} & +(h \bar{v})_{y}=0 \\
\bar{u}_{t}+\bar{u}_{x} & +\bar{v}_{y}+g \eta_{x}-(1 / 3) h_{r}^{2}\left(\bar{u}_{x x t}+\bar{v}_{x y t}\right)+h_{r} \xi_{x} \bar{u}_{x t} \\
& +(1 / 2) h_{r}\left(\xi_{x x} \bar{u}_{t}+\xi_{y} \bar{v}_{x t}+\xi_{x} \bar{v}_{y t}+\xi_{x y} \bar{v}_{t}\right)=0 \\
\bar{v}_{t}+\bar{u}_{x} & +\overline{v v}_{y}+g \eta_{y}-(1 / 3) h_{r}^{2}\left(\bar{u}_{x y t}+\bar{v}_{y y t}\right)+h_{r} \xi_{y} \bar{v}_{y t} \\
& +(1 / 2) h_{r}\left(\xi_{y y} \bar{v}_{t}+\xi_{y} \bar{u}_{x t}+\xi_{x} \bar{u}_{y t}+\xi_{x y} \bar{u}_{t}\right)=0
\end{aligned}
$$

Further simplifying the equations of motion (18), retaining only terms up to order 1 in $\sigma$, i.e., neglecting all terms of dispersive origin, this system of equations is written in dimensional variables:

$$
\begin{aligned}
& h_{t}+(h \bar{u})_{x}+(h \bar{v})_{y}=0 \\
& \bar{u}_{t}+\bar{u} \bar{u}_{x}+\bar{v} \bar{u}_{y}+g \eta_{x}=0 \\
& \bar{v}_{t}+\bar{u}_{x}+\bar{v}_{y}+g \eta_{y}=0
\end{aligned}
$$

Approaches (19), (24) and (25) are known as Serre equations, or Green \& Naghdi, Boussinesq and Saint-Venant, respectively, in two horizontal dimensions (2HD models). The classical Serre equations (19) [17] are fully nonlinear and weakly dispersive. Boussinesq equations (24) only incorporate weak dispersion and weak nonlinearity and are valid only for long waves in shallow waters. As for the Boussinesq-type models, also Serre's equations are valid only for shallow water conditions.

\section{Derivation of higher-order equations}

\subsection{Weakly nonlinear approaches with improved dispersive performance}

\subsubsection{Nwogu's approach}

To allow applications in a greater range of $h_{0} / \lambda$, other than shallow waters, [27] introduced higher-order dispersive terms into the governing equations to improve linear dispersion 
properties. By redefining the dependent variable, [30] achieved the same improvement without the need to add such terms to the equations.

Following [36], the extended Bussinesq equations obtained by [30] are derived in this section, using the nondimensionalised scaled equation system (2) - (3) as the starting point, rather than the procedure presented by Nwogu. For simplicity reasons, and without loss of generality, the one-dimensional extended Boussinesq equation system is derived. For consistency with the previous work (Section 2), the continuity equation 2(a) in two vertical dimensions $(x, z)$ is integrated through the depth:

$$
\begin{gathered}
\int_{-1+\xi}^{z} w_{z} d z=-\int_{-1+\xi}^{z} u_{x} d z \\
\left.w\right|_{z}-\left.w\right|_{-1+\xi}=-\left(\int_{-1+\xi}^{z} u d z\right)_{x}+\left.u\right|_{-1+\xi} \xi_{x}-\left.u\right|_{z} z_{x}
\end{gathered}
$$

Denoting $\left.w\right|_{z}$ by $w$, with $\xi_{t}=0$ and using Leibnitz' rule, the boundary condition 3(a) at $z=-1+\xi$ gives:

$$
w=-\left(\int_{-1+\xi}^{z} u d z\right)_{x}
$$

Substituting (28) in the irrotationality equation $2(\mathrm{e}), u_{z}=\sigma^{2} w_{x}$, yields:

$$
u_{z}=\sigma^{2} w_{x}=-\sigma^{2}\left(\int_{-1+\xi}^{z} u d z\right)_{x x}
$$

Considering a Taylor series expansion (30) of $u(x, z, t)$, about $z=z_{\alpha}$, where the horizontal velocity $u_{\alpha}(x, t)$ denotes the velocity at depth $z_{\alpha}, u\left(x, z_{\alpha}, t\right)$, this Taylor expansion is integrated through the depth from $-1+\xi$ to $z$, yielding (31) (for details see [36]):

$$
\begin{gathered}
u=u_{\alpha}+\left.\left(z-z_{\alpha}\right) u_{z}\right|_{z=z_{\alpha}}+\left.\frac{\left(z-z_{\alpha}\right)^{2}}{2} u_{z z}\right|_{z=z_{\alpha}}+\cdots \\
\int_{-1+\xi}^{z} u d z=(z+1-\xi) u_{\alpha}+\left.\left[\frac{\left(z-z_{\alpha}\right)^{2}}{2}-\frac{\left(1-\xi+z_{\alpha}\right)^{2}}{2}\right] u_{z}\right|_{z=z_{\alpha}} \\
+\left.\left[\frac{\left(z-z_{\alpha}\right)^{3}}{6}+\frac{\left(1-\xi+z_{\alpha}\right)^{3}}{6}\right] u_{z z}\right|_{z=z_{\alpha}}+\cdots
\end{gathered}
$$


Substituting (31) in equation (29) gives:

$$
u_{z}=-\sigma^{2}\left\{(z+1-\xi) u_{\alpha}+\left.\left[\frac{\left(z-z_{\alpha}\right)^{2}}{2}-\frac{\left(1-\xi+z_{\alpha}\right)^{2}}{2}\right] u_{z}\right|_{z=z_{\alpha}}+\cdots\right\}_{x x}
$$

Differentiating equation (32) with respect to $z$, noting from (29) that $u_{z}=O\left(\sigma^{2}\right)$ :

$$
\begin{aligned}
u_{z z} & =-\sigma^{2}\left\{u_{\alpha}+\left.\left(z-z_{\alpha}\right) u_{z}\right|_{z=z_{\alpha}}+\left.\frac{\left(z-z_{\alpha}\right)^{2}}{2} u_{z z}\right|_{z=z_{\alpha}}+\cdots\right\}_{x x} \\
& =-\sigma^{2} u_{\alpha x x}+O\left(\sigma^{4}\right)
\end{aligned}
$$

Differentiating equation (33) with respect to $z$ and noting that both $u_{z}$ and $u_{z z}$ are $O\left(\sigma^{2}\right)$ :

$$
u_{z z z}=-\sigma^{2}\left\{\left.u_{z}\right|_{z=z_{\alpha}}+\left.\left(z-z_{\alpha}\right) u_{z z}\right|_{z=z_{\alpha}}+\cdots\right\}_{x x}=O\left(\sigma^{4}\right)
$$

Repeated differentiation of this expression will produce expressions for the higher derivatives of $u$ with respect to $\mathrm{z}$ and show them to be of $O\left(\sigma^{4}\right)$ order or greater. Substituting equations (33) and (34) back in equation (32):

$$
\begin{aligned}
u_{z} & =-\sigma^{2}\left\{\left(1-\xi+z_{\alpha}\right) u_{\alpha}-\left.\frac{\left(1-\xi+z_{\alpha}\right)^{2}}{2} u_{z}\right|_{z=z_{\alpha}}+O\left(\sigma^{2}\right)\right\}_{x x} \\
& =-\sigma^{2}\left[\left(1-\xi+z_{\alpha}\right) u_{\alpha}\right]_{x x}+O\left(\sigma^{4}\right)
\end{aligned}
$$

Substituting equations (33), (34) and (35) in the Taylor series expansion (30) produces an expression for the horizontal velocity component $u$ :

$$
u=u_{\alpha}-\sigma^{2}\left\{\left(z-z_{\alpha}\right)\left[\left(1-\xi+z_{\alpha}\right) u_{\alpha}\right]_{x x}+\frac{\left(z-z_{\alpha}\right)^{2}}{2}\left(u_{\alpha}\right)_{x x}\right\}+O\left(\sigma^{4}\right)
$$

Substituting the horizontal velocity (36) in equation (28) leads to an expression for the vertical velocity component $w$ : 


$$
\begin{aligned}
w= & -\left\{\left(1-\xi+z_{\alpha}\right) u_{\alpha}\right. \\
& \left.+\sigma^{2}\left[\frac{\left(1-\xi+z_{\alpha}\right)^{2}}{2}\left[\left(1-\xi+z_{\alpha}\right) u_{\alpha}\right]_{x x}-\frac{\left(1-\xi+z_{\alpha}\right)^{3}}{6}\left(u_{\alpha}\right)_{x x}\right]\right\}_{x} \\
& +O\left(\sigma^{4}\right)
\end{aligned}
$$

Using the velocities $u(36)$ and $w(37)$ in the vertical momentum, equation 2(d) yields:

$$
\varepsilon \sigma^{2}\left\{-\left[(z+1-\xi) u_{\alpha}\right]_{x t}+O\left(\sigma^{2}\right)\right\}+p_{z}+1+O\left(\varepsilon^{2} \sigma^{2}\right)=0
$$

This can be rearranged to give an expression for the pressure $p$ :

$$
-p_{z}=1-\varepsilon \sigma^{2}\left[(z+1-\xi) u_{\alpha}\right]_{x t}+O\left(\varepsilon \sigma^{4}, \varepsilon^{2} \sigma^{2}\right)
$$

Integrating through the depth from $z$ to the free surface $\varepsilon \eta$ :

$$
-\left.p\right|_{\varepsilon \eta}+\left.p\right|_{z}=\varepsilon \eta-z+\varepsilon \sigma^{2}\left\{\left[\frac{z^{2}}{2}+(1-\xi) z\right] u\right\}_{x t}+O\left(\varepsilon \sigma^{4}, \varepsilon^{2} \sigma^{2}\right)
$$

Using the free surface boundary condition 3(c) for the pressure, and denoting $\left.p\right|_{z}$ simply as $p$ gives:

$$
p=\varepsilon \eta-z+\varepsilon \sigma^{2}\left\{\left[\frac{z^{2}}{2}+(1-\xi) z\right] u\right\}_{x t}+O\left(\varepsilon \sigma^{4}, \varepsilon^{2} \sigma^{2}\right)
$$

Substituting the equations for the horizontal velocity component (36), the vertical velocity component (37) and the pressure (41) in the horizontal momentum equation 2(b) gives the Boussinesq momentum equation:

$$
\begin{aligned}
u_{\alpha t}-\sigma^{2}\left\{\left(z-z_{\alpha}\right)\left[\left(1-\xi+z_{\alpha}\right) u_{\alpha}\right]_{x x}+\frac{\left(z-z_{\alpha}\right)^{2}}{2}\left(u_{\alpha}\right)_{x x}\right\}_{t} \\
+\varepsilon u_{\alpha} u_{\alpha x}+\eta_{x}+\sigma^{2}\left\{\left[\frac{z^{2}}{2}+(1-\xi) z\right] u_{\alpha}\right\}_{x x t}=O\left(\varepsilon \sigma^{2}, \sigma^{4}\right)
\end{aligned}
$$


or

$$
u_{\alpha t}+\varepsilon u_{\alpha} u_{\alpha x}+\eta_{x}+\sigma^{2}\left\{z_{\alpha}\left[(1-\xi) u_{\alpha}\right]_{x x}+\frac{z_{\alpha}^{2}}{2}\left(u_{\alpha}\right)_{x x}\right\}_{t}=O\left(\varepsilon \sigma^{2}, \sigma^{4}\right)
$$

The second equation of the Boussinesq system is developed by first integrating the continuity equation 2(a) through the depth $-1+\xi$ :

$$
\left(\int_{-1+\xi}^{\varepsilon \eta} u d z\right)_{x}-\left.\varepsilon u\right|_{\varepsilon \eta} \eta_{x}+\left.u\right|_{-1+\xi} \xi_{x}+\left.w\right|_{\varepsilon \eta}-\left.w\right|_{-1+\xi}=0
$$

Using Liebnitz' Rule and the kinematic boundary conditions at the bed $z=-1+\xi 3$ (a) and at the free surface $z=\varepsilon \eta 3(\mathrm{~b})$ gives:

$$
\eta_{t}+\left(\int_{-1+\xi}^{\varepsilon \eta} u d z\right)_{x}=0
$$

From the expression (36) for the horizontal velocity $u$ :

$$
\begin{aligned}
\int_{-1+\xi}^{\varepsilon \eta} u d z=(1-\xi+\varepsilon \eta) u_{\alpha}- & \sigma^{2}\left\{\left[\frac{\left(z-z_{\alpha}\right)^{2}}{2}\right]_{-1+\xi}^{\varepsilon \eta}\left[\left(1-\xi+z_{\alpha}\right) u_{\alpha}\right]_{x x}\right. \\
+ & {\left.\left[\frac{\left(z-z_{\alpha}\right)^{3}}{6}\right]_{-1+\xi}^{\varepsilon \eta} u_{\alpha x x}\right\}+O\left(\sigma^{4}\right) }
\end{aligned}
$$

or

$$
\begin{gathered}
\int_{-1+\xi}^{\varepsilon \eta} u d z=(1-\xi+\varepsilon \eta) u_{\alpha}-\sigma^{2}\left\{\left[\frac{z_{\alpha}^{2}}{2}-\frac{\left(1-\xi+z_{\alpha}\right)^{2}}{2}\right]\left[\left(1-\xi+z_{\alpha}\right) u_{\alpha}\right]_{x x}\right. \\
\left.+\left[-\frac{z_{\alpha}^{3}}{6}+\frac{\left(1-\xi+z_{\alpha}\right)^{3}}{6}\right]_{\alpha x x}\right\}+O\left(\varepsilon \sigma^{2}, \sigma^{4}\right)
\end{gathered}
$$

yielding: 


$$
\begin{aligned}
\int_{-1+\xi}^{\varepsilon \eta} u d z= & (1-\xi+\varepsilon \eta) u_{\alpha}-\sigma^{2}\left\{\left[-\frac{(1-\xi)^{2}}{2}+(1-\xi) z_{\alpha}\right]\left[\left(1-\xi+z_{\alpha}\right) u_{\alpha}\right]_{x x}\right. \\
& \left.+\left[\frac{(1-\xi)^{3}}{6}+\frac{(1-\xi)^{2} z_{\alpha}}{2}+\frac{(1-\xi) z_{\alpha}^{2}}{2}\right]\left(u_{\alpha}\right)_{x x}\right\}+O\left(\varepsilon \sigma^{2}, \sigma^{4}\right)
\end{aligned}
$$

and thus

$$
\begin{array}{r}
\int_{-1+\xi}^{\varepsilon \eta} u d z=(1-\xi+\varepsilon \eta) u_{\alpha}+\sigma^{2}\left\{\left[z_{\alpha}+\frac{1-\xi}{2}\right](1-\xi)\left[(1-\xi) u_{\alpha}\right]_{x x}\right. \\
\left.+\left[\frac{z_{\alpha}^{2}}{2}-\frac{(1-\xi)^{2}}{6}\right](1-\xi)\left(u_{\alpha}\right)_{x x}\right\}+O\left(\varepsilon \sigma^{2}, \sigma^{4}\right)
\end{array}
$$

Substituting equation (49) in the free surface equation (45) gives the Boussinesq continuity equation:

$$
\begin{gathered}
\eta_{t}+\left[(1-\xi+\varepsilon \eta) u_{\alpha}\right]_{x}+\sigma^{2}\left\{\left(z_{\alpha}+\frac{1-\xi}{2}\right)(1-\xi)\left[(1-\xi) u_{\alpha}\right]_{x x}\right. \\
\left.+\left(\frac{z_{\alpha}^{2}}{2}-\frac{(1-\xi)^{2}}{6}\right)(1-\xi)\left(u_{\alpha}\right)_{x x}\right\}_{x}=O\left(\varepsilon \sigma^{2}, \sigma^{4}\right)
\end{gathered}
$$

Returning to dimensional variables, unscaled form, equations (43) and (50) are written:

$$
\begin{gathered}
u_{\alpha t}+u_{\alpha} u_{\alpha x}+g \eta_{x}+z_{\alpha}\left(h_{r} u_{\alpha t}\right)_{x x}+\frac{z_{\alpha}^{2}}{2}\left(u_{\alpha}\right)_{x x t}=0 \\
\eta_{t}+\left[\left(h_{0}+\eta\right) u_{\alpha}\right]_{x}+\left[\left(z_{\alpha}+\frac{h_{r}}{2}\right) h_{r}\left(h_{r} u_{\alpha}\right)_{x x}+\left(\frac{z_{\alpha}^{2}}{2}-\frac{h_{r}^{2}}{6}\right) h_{r}\left(u_{\alpha}\right)_{x x}\right]_{x}=0
\end{gathered}
$$

Setting the arbitrary depth $z_{\alpha}=\alpha h_{0}$, where $-1 \leq \alpha \leq 0$, the system (51)-(52) is rewritten:

$$
u_{\alpha t}+u_{\alpha} u_{\alpha x}+g \eta_{x}+\alpha h_{r}\left[h_{r}\left(u_{\alpha}\right)_{t}\right]_{x x}+\frac{\alpha^{2}}{2} h_{r}^{2}\left(u_{\alpha}\right)_{x x t}=0
$$




$$
\eta_{t}+\left[\left(h_{r}+\eta\right) u_{\alpha}\right]_{x}+\left[\left(\alpha+\frac{1}{2}\right) h_{r}^{2}\left(h_{r} u_{\alpha}\right)_{x x}+\left(\frac{\alpha^{2}}{2}-\frac{1}{6}\right) h_{r}^{3}\left(u_{\alpha}\right)_{x x}\right]_{x}=0
$$

In two dimensions, the equation system (53)-(54) is written:

$$
\begin{aligned}
\eta_{t} & +\nabla \cdot\left[\left(h_{r}+\eta\right) \boldsymbol{u}_{\alpha}\right] \\
& +\nabla\left\{\left(\alpha+\frac{1}{2}\right) h_{r}^{2} \nabla\left[\nabla \cdot\left(h_{r} \boldsymbol{u}_{\alpha}\right)\right]+\left(\frac{\alpha^{2}}{2}-\frac{1}{6}\right) h_{r}^{3} \nabla\left(\nabla \cdot \boldsymbol{u}_{\alpha}\right)\right\}=0 \\
\boldsymbol{u}_{\alpha t} & +\left(\boldsymbol{u}_{\alpha} \cdot \nabla\right) \boldsymbol{u}_{\alpha}+g \nabla \eta+\alpha h_{r} \nabla\left[\nabla \cdot\left(h_{r} \boldsymbol{u}_{\alpha t}\right)\right]+\frac{\alpha^{2}}{2} h_{r}^{2} \nabla\left(\nabla \cdot \boldsymbol{u}_{\alpha t}\right)=0
\end{aligned}
$$

where $\nabla$ represents the two-dimensional gradient operator with respect to the horizontal coordinates $(x, y)(\nabla=\partial / \partial x, \partial / \partial y)$, and the velocity vector $u(x, y, t)=(u, v)$ represents the twodimensional velocity field at depth $z=\alpha h$.

\subsubsection{Beji and Nadaoka's, and Liu and Sun's approaches}

Starting from the standard Boussinesq equations and adopting the methodology introduced by [27] [11] presented a new approach that allows for applications until values of $h_{0} / \lambda$ to the order of 0.25 , and still with acceptable errors in amplitude and phase velocity up to values of $h_{0} / \lambda$ near 0.50 . By an addition and subtraction process, using the approximation $u_{t}=-g \eta_{x}$ and considering a dispersion parameter $\beta$ in the momentum equation of the 1HD system (24), Beji and Nadaoka obtained an improved set of Boussinesq equations for variable depth, with $\beta$ value obtained by comparison of the dispersion relation of the linearized form of the resulting equation with a second-order Padé expansion of the linear dispersion relation $\omega^{2} / g k=\tanh \left(k h_{r}\right)$. In order to improve dispersion and linear shoaling characteristics in the Beji and Nadaoka's equations, [23] introduced two tuning parameters, $\alpha$ and $\gamma$, so that $\beta=1.5 \alpha-0.5 \gamma$. The nonlinearity in the previous Boussinesq-type models was improved by Liu and Sun adding higher-order terms accurate to the order of $O\left(\varepsilon \sigma^{2}\right)$. The 1HD standard Boussinesq equations and the approaches of Beji and Nadaoka and Liu and Sun are identified within the following system of equations (57) for water of variable depth:

$$
\begin{aligned}
& h_{t}+(h u)_{x}=0 \\
& u_{t}+u u_{x}+g \eta_{x}-\{(1+\alpha) / 2-(1+\gamma) / 6\} h_{r}^{2} u_{x x t} \\
& \quad-(\alpha / 2-\gamma / 6) g h_{r}^{2} \eta_{x x x}+(1+\alpha) h_{r} \xi_{x} u_{x t}+\alpha g h_{r} \xi_{x} \eta_{x x} \\
& \quad+0.5(1+\alpha) h_{r} \xi_{x x} u_{t}+0.5 \alpha g h_{r} \xi_{x x} \eta_{x}=O\left(\varepsilon \sigma^{2}\right)
\end{aligned}
$$


After linearization of the equations system (57), [23] obtained the following dispersion relation (58):

$$
\frac{\omega^{2}}{g k}=\frac{k h_{r}\left[1+(\alpha / 2-\gamma / 6) k^{2} h_{r}^{2}\right]}{1+(1+\alpha) k^{2} h_{r}^{2} / 2-(1+\gamma) k^{2} h_{r}^{2} / 6}
$$

Comparing equation (58), written in terms of the phase speed (59)

$$
C^{2}=\frac{\omega^{2}}{k^{2}}=g h_{r}\left[\frac{1+(\alpha / 2-\gamma / 6)\left(k h_{r}\right)^{2}}{1+(1+\alpha)\left(k h_{r}\right)^{2} / 2-(1+\gamma)\left(k h_{r}\right)^{2} / 6}\right]
$$

with the linear dispersion relation $\omega^{2} / g k=\tanh \left(k h_{r}\right)$, using the approach (60)

$$
C_{\text {Airy }}^{2}=\frac{\omega^{2}}{k^{2}}=\left(g h_{r}\right) \tanh \left(k h_{r}\right)=g h_{r}\left[\frac{1+\left(k h_{r}\right)^{2} / 15}{1+2\left(k h_{r}\right)^{2} / 5}\right]+O\left[(k h)^{6}\right]
$$

allows to obtain the best values for the parameters $\alpha$ and $\gamma: \alpha=0.1308$ and $\gamma=-0.0076$.

Considering appropriate values for the tuning parameters $\alpha$ and $\gamma$, we can identify within the equation system (57):

- The standard Boussinesq equations by setting $\alpha=\gamma=0$

- The Beji and Nadaoka equations considering $\alpha=\gamma=0.20$

- The Liu and Sun equations with $\alpha=0.1308$ and $\gamma=-0.0076$

A visual comparison of numerical results of the extended Boussinesq approximation (57), with $\alpha=0.1308$ and $\gamma=-0.0076)$, shown in [1,2] and [3], with a similar study performed by [37], using the extended Boussinesq (53)-(54) model (Nwogu's approach, with $\alpha=-0.531$ ) shows no relevant differences in the graphs. In this regard, it is worth remembering [4] "although the methods of derivation are different, the resulting dispersion relations of these extended Boussinesq equations are similar, and may be thought of as a slight modification of the second-order Padé approximant of the full dispersion relation".

\subsection{Fully nonlinear approaches with improved dispersive performance}

Instead of using the horizontal velocity at a certain depth, other extensions of the Boussinesq equations have been made by using the velocity potential on an arbitrary depth, also with a tuning parameter. Wei et al. (1995) [39] used the Nwogu's approach to derive a Boussinesqtype model which retains full nonlinearity by including $O\left(\varepsilon \sigma^{2}\right)$ terms not considered in the 
Nwogu's (53)-(54) system, and thus improving the nonlinearity to $O(\varepsilon)=1$. Wei et al., and later [16] Gobbi et al., derived a fourth-order Boussinesq model in which the velocity potential is approximated by a fourth-order polynomial in $z$. In terms of non-dimensional variables, both consider the boundary value problem for potential flow, given by:

$$
\begin{array}{ll}
\varphi_{z z}+\mu^{2} \nabla^{2} \varphi=0, & -1+\xi \leq z \leq \varepsilon \eta \\
\varphi_{z}+\mu^{2} \nabla h_{1-\xi} \bullet \nabla \varphi=0, & z=-1+\xi \\
\eta+\varphi_{t}+\frac{1}{2} \varepsilon\left[(\nabla \varphi)^{2}+\frac{1}{\mu^{2}}\left(\varphi_{z}\right)^{2}\right]=0, & z=\varepsilon \eta \\
\eta_{t}+\varepsilon \nabla \varphi \cdot \nabla \eta-\frac{1}{\mu^{2}} \varphi_{z}=0, & z=\varepsilon \eta
\end{array}
$$

where, as above, $z$ is the vertical coordinate starting at the still water level $h_{0}(x, y)$ and pointing upwards, scaled by a typical depth $h_{0}$, and $\eta$ is the water surface displacement scaled by a representative amplitude $a$. The two dimensionless parameters $\varepsilon$ and $\mu^{2}$ are defined as $\varepsilon=a / h_{0}$ and $\mu^{2}=\left(k_{0} h_{0}\right)^{2}$, with a representative wave number $k_{0}=2 \pi / \lambda$, so $\mu^{2}=(2 \pi \sigma)^{2}$. Time $t$ is scaled by $\left[k_{0}\left(g h_{0}\right)^{1 / 2}\right]^{-1}$, and $\varphi$, the velocity potential, is scaled by $\varepsilon h_{0}\left(g h_{0}\right)^{1 / 2}$. We use the nondimensional water level $h_{1-\xi}$ instead of $1-\xi$. Integrating the first equation of (61) over the water column, and using the appropriate boundary conditions, the continuity equation is obtained:

$$
\eta_{t}+\nabla \cdot M=0
$$

where $M=\int_{-1+\xi}^{\varepsilon \eta} \nabla \varphi d z$. Retaining terms to $O\left(\mu^{2}\right)$, and denoting $\varphi_{\alpha}$ as the value of $\varphi$ at $z=z_{\alpha}(x, y)$, an approximate velocity potential is given by:

$$
\varphi=\varphi_{\alpha}(x, t)+\mu^{2}\left(z_{\alpha}-z\right) \nabla \cdot\left(h_{1-\xi} \nabla \varphi_{\alpha}\right)+\frac{\mu^{2}}{2}\left(z_{\alpha}^{2}-z^{2}\right) \nabla^{2} \varphi_{\alpha}+O\left(\mu^{4}\right)
$$

Substituting equation (63) into (62), a mass flux conservation equation is obtained [39]:

$$
\begin{aligned}
\eta_{t} & +\nabla \cdot\left\{( h _ { 1 - \xi } + \varepsilon \eta ) \left\{\nabla \varphi_{\alpha}+\mu^{2}\left\{\nabla\left[z_{\alpha} \nabla \cdot\left(h_{1-\xi} \nabla \varphi_{\alpha}\right)+\frac{z_{\alpha}^{2}}{2} \nabla^{2} \varphi_{\alpha}\right]\right.\right.\right. \\
& \left.\left.\left.+\frac{\left(h_{1-\xi}-\varepsilon \eta\right)}{2} \nabla\left[\nabla \cdot\left(h_{1-\xi} \nabla \varphi_{\alpha}\right)\right]-\frac{\left[h_{1-\xi}^{2}-\varepsilon \eta h_{1-\xi}+(\varepsilon \eta)^{2}\right]}{6} \nabla^{2} \nabla \varphi_{\alpha}\right\}\right\}\right\}=0
\end{aligned}
$$


Similarly, substituting (63) into the third equation of (61), a momentum equation is obtained in terms of the velocity potential. Then, given that at $z=z_{\alpha}, u_{\alpha}=\nabla \varphi_{\alpha^{\prime}}$ a fully nonlinear version of a Boussinesq type model in terms of $\eta$ and $u_{\alpha}$ is written:

$$
\begin{gathered}
\eta_{t}+\nabla \cdot\left\{( h _ { 1 - \xi } + \varepsilon \eta ) \left\{\boldsymbol{u}_{\alpha}+\mu^{2}\left\{\left[\frac{z_{\alpha}^{2}}{2}-\frac{1}{6}\left(h_{1-\xi}^{2}-\varepsilon \eta h_{1-\xi}+(\varepsilon \eta)^{2}\right)\right] \nabla\left(\nabla \cdot \boldsymbol{u}_{\alpha}\right)\right.\right.\right. \\
\left.\left.\left.+\left[z_{\alpha}+\frac{1}{2}\left(h_{1-\xi}-\varepsilon \eta\right)\right] \nabla\left[\nabla \cdot\left(h_{1-\xi} \boldsymbol{u}_{\alpha}\right)\right]\right\}\right\}\right\}=O\left(\mu^{4}\right) \\
\boldsymbol{u}_{\alpha t}+\varepsilon\left(\boldsymbol{u}_{\alpha} \cdot \nabla\right) \boldsymbol{u}_{\alpha}+\nabla \eta+\mu^{2} \boldsymbol{R}+\varepsilon \mu^{2} \boldsymbol{S}=O\left(\mu^{4}\right)
\end{gathered}
$$

where

$$
\begin{gathered}
\boldsymbol{R}=\frac{1}{2} z_{\alpha}^{2} \nabla\left(\nabla \cdot \boldsymbol{u}_{\alpha t}\right)+z_{\alpha} \nabla\left[\nabla \cdot\left(h_{1-\xi} \boldsymbol{u}_{\alpha t}\right)\right]-\nabla\left[\frac{1}{2}(\varepsilon \eta)^{2} \nabla \cdot \boldsymbol{u}_{\alpha t}+\varepsilon \eta \nabla \cdot\left(h_{1-\xi} \boldsymbol{u}_{\alpha t}\right)\right] \\
\boldsymbol{S}=\nabla\left\{\left(z_{\alpha}-\varepsilon \eta\right)\left(\boldsymbol{u}_{\alpha} \cdot \nabla\right)\left[\nabla \cdot\left(h_{1-\xi} \boldsymbol{u}_{\alpha}\right)\right]+\frac{1}{2}\left[z_{\alpha}^{2}-(\varepsilon \eta)^{2}\right]\left(\boldsymbol{u}_{\alpha} \cdot \nabla\right)\left(\nabla \cdot \boldsymbol{u}_{\alpha}\right)\right\} \\
+\frac{1}{2} \nabla\left\{\left[\nabla \cdot\left(h_{1-\xi} \boldsymbol{u}_{\alpha}\right)+\varepsilon \eta \nabla \cdot \boldsymbol{u}_{\alpha}\right]^{2}\right\}
\end{gathered}
$$

It should be noted that Nwogu's approximation is recovered by neglecting higher-order terms. Numerical computations show that this model agrees well with solutions of the full potential problem over the range of relevant water depths.

A high-order, predictor-corrector, finite-difference numerical algorithm to solve this model was developed and is presented in [25] for the one- and two-layer models (see Sections 3.2.1 and 3.2.2). Considering one layer only, the corresponding numerical model that is the basis of the COULWAVE model is presented later, in Sections 4.1.3 and 4.1.4.

To improve the accuracy of numerical models, it has been common practice the use of highorder polynomials to approximate the vertical velocity dependence. However, this requires very elaborate and expensive numerical calculation procedures. A different approach is suggested by [26], which consists of using quadratic polynomials, matched at interfaces that divide the water column into layers. In this regard, it is worth mentioning [26] "this approach leads to a set of model equations without the high-order spatial derivatives associated with high-order polynomial approximations". 


\subsubsection{Mathematical model for one-layer}

Defining the parameters $S_{1}=\nabla \cdot u_{1}$ and $T_{1}=\nabla \cdot\left[(1-\xi) u_{1}\right]+(1 / \varepsilon)\left(\partial h_{1-\xi} / \partial t\right)$, the model uses the following approach for the continuity equation (to compute $\eta$ values), in nondimensional variables:

$$
\begin{aligned}
\frac{1}{\varepsilon} \frac{\partial h_{1-\xi}}{\partial t}+\frac{\partial \eta}{\partial t}+\nabla \cdot & {\left[\left(\varepsilon \eta+h_{1-\xi}\right) \boldsymbol{u}_{1}\right] } \\
& -\mu^{2} \nabla \cdot\left\{\left[\frac{\varepsilon^{3} \eta^{3}+h_{1-\xi}^{3}}{6}-\frac{\left(\varepsilon \eta+h_{1-\xi}\right) k_{1}^{2}}{2}\right] \nabla S_{1}\right. \\
& \left.+\left[\frac{\varepsilon^{2} \eta^{2}-h_{1-\xi}^{2}}{2}-\left(\varepsilon \eta+h_{1-\xi}\right) k_{1}\right] \nabla T_{1}\right\}=O\left(\mu^{4}\right)
\end{aligned}
$$

where $u_{1}=$ horizontal velocity vector, $k_{1}=\alpha_{1} h+\beta_{1} \eta, \alpha_{1}$ and $\beta_{1}$ are coefficients to be defined by the user. The index 1 means one-layer model. To compute the velocity components $(u, v)$, the following approach of the momentum equation is solved, in nondimensional variables:

$$
\begin{aligned}
\frac{\partial \boldsymbol{u}_{1}}{\partial t}+ & \varepsilon \boldsymbol{u}_{1} \cdot \nabla \boldsymbol{u}_{1}+\nabla \eta+\mu^{2} \frac{\partial}{\partial t}\left\{\frac{k_{1}^{2}}{2} \nabla S_{1}+k_{1} \nabla T_{1}\right\} \\
& +\varepsilon \mu^{2}\left[\left(\boldsymbol{u}_{1} \cdot \nabla k_{1}\right) \nabla T_{1}+k_{1} \nabla\left(\boldsymbol{u}_{1} \cdot \nabla T_{1}\right)+k_{1}\left(\boldsymbol{u}_{1} \cdot \nabla k_{1}\right) \nabla S_{1}\right. \\
& \left.+\frac{k_{1}^{2}}{2} \nabla\left(\boldsymbol{u}_{1} \cdot \nabla S_{1}\right)\right]+\varepsilon \mu^{2}\left[T_{1} \nabla T_{1}-\nabla\left(\eta \frac{\partial T_{1}}{\partial t}\right)\right] \\
& +\varepsilon^{2} \mu^{2} \nabla\left(\eta S_{1} T_{1}-\frac{\eta^{2}}{2} \frac{\partial S_{1}}{\partial t}-\eta \boldsymbol{u}_{1} \cdot \nabla T_{1}\right) \\
& +\varepsilon^{2} \mu^{2} \nabla\left[\frac{\eta^{2}}{2}\left(S_{1}^{2}-\boldsymbol{u}_{1} \cdot \nabla S_{1}\right)\right]=O\left(\mu^{4}\right)
\end{aligned}
$$

The horizontal velocity vector is given as:

$$
\boldsymbol{U}_{1}=\boldsymbol{u}_{1}-\mu^{2}\left\{\frac{z_{1}^{2}-k_{1}^{2}}{2} \nabla S_{1}+\left(z_{1}-k_{1}\right) \nabla T_{1}\right\}+O\left(\mu^{4}\right)
$$

This one-layer model, often referred to as the "fully nonlinear, extended Boussinesq equations" in the literature (e.g. [38]), has been examined and applied to a significant extent. The weakly nonlinear version of (69)-(71) (i.e., assuming $O(\varepsilon)=O\left(\mu^{2}\right)$, thereby neglecting all nonlinear dispersive terms) was first derived by [30]. Through a linear and first-order nonlinear analysis of the model equations, Nwogu recommended that $z_{1}=-0.531 h$, and that value has been 
recommended and adopted by most researchers who use these equations. Refs. [22] and later [38] used the Nwogu's approach to derive a high-order model which retains "full nonlinearity" by including terms not considered in the Nwogu's approach. Comparing Liu's and Wei and Kirby's equations, there are some differences that can be attributed to the absence of some nonlinear dispersive terms in Wei and Kirby [19]. The above one-layer equations (69) and (70) are identical to those derived by [22].

\subsubsection{Mathematical model for two-layers}

Details about the two-layer mathematical model are presented in [25] and [24]. The model consists of a continuity equation, a momentum equation for the upper layer and a matching equation for the velocity in the lower layer. These equations are solved using an appropriate numerical method to compute values for the free surface elevation $\eta$ and the velocity components $(u, v)$. In dimensional variables, this set of equations is written ([24]):

$$
\begin{aligned}
\frac{\partial \eta}{\partial t}+\nabla \cdot & {\left[(\eta-\zeta) \boldsymbol{u}_{1}+\left(\zeta+h_{r}\right) \boldsymbol{u}_{2}\right] } \\
- & \nabla \cdot\left\{\left[\frac{\zeta^{3}+h_{r}^{3}}{6}-\frac{\left(\zeta+h_{r}\right) k_{2}^{2}}{2}\right] \nabla S_{2}+\left[\frac{\zeta^{2}-h_{r}^{2}}{2}-\left(\zeta+h_{r}\right) k_{2}\right] \nabla T_{2}\right\} \\
- & \nabla \cdot\left\{\left[\frac{\eta^{3}-\zeta^{3}}{6}-\frac{(\eta-\zeta) k_{1}^{2}}{2}\right] \nabla S_{1}+\left[\frac{\eta^{2}-\zeta^{2}}{2}-(\eta-\zeta) k_{1}\right] \nabla T_{1}\right\}=0 \\
\frac{\partial \boldsymbol{u}_{1}}{\partial t}+ & \frac{1}{2} \nabla\left(\boldsymbol{u}_{1} \cdot \boldsymbol{u}_{1}\right)+g \nabla \eta \\
& +\frac{\partial}{\partial t}\left[\frac{k_{1}^{2}}{2} \nabla S_{1}+k_{1} \nabla T_{1}-\nabla\left(\frac{\eta^{2}}{2} S_{1}\right)-\nabla\left(\eta T_{1}\right)\right] \\
+ & \nabla\left[\frac{\partial \eta}{\partial t}\left(T_{1}+\eta S_{1}\right)+\left(k_{1}-\eta\right)\left(\boldsymbol{u}_{1} \cdot \nabla\right) T_{1}+\frac{1}{2}\left(k_{1}^{2}-\eta^{2}\right)\left(\boldsymbol{u}_{1} \cdot \nabla\right) S_{1}\right. \\
& \left.+\frac{1}{2}\left(T_{1}+\eta S_{1}\right)^{2}\right]-R_{b}+R_{f}+v_{T}\left[\nabla S_{1}-\nabla^{2} \boldsymbol{u}_{1}\right. \\
& \left.-\nabla^{2}\left(\frac{k_{1}^{2}}{2} \nabla S_{1}+k_{1} \nabla T_{1}\right)+\nabla\left(\frac{\eta^{2}}{2} \nabla^{2} S_{1}+\eta \nabla^{2} T_{1}\right)\right]=0 \\
\boldsymbol{u}_{2}+ & \frac{k_{2}^{2}-\zeta^{2}}{2} \nabla S_{2}+\left(k_{2}-\zeta\right) \nabla T_{2}=u_{1}+\frac{k_{1}^{2}-\zeta^{2}}{2} \nabla S_{1}+\left(k_{1}-\zeta\right) \nabla T_{1}
\end{aligned}
$$

where $S_{1}=\nabla \cdot u_{1}, T_{1}=\zeta\left(S_{2}-S_{1}\right)+T_{2}, S_{2}=\nabla \cdot u_{2}$, and $T_{2}=\nabla .\left(h_{r} u_{2}\right) \cdot R_{b}=$ breaking-related dissipation term, $R_{f}=\left[f /\left(h_{r}+\eta\right)\right] \boldsymbol{u}_{b}\left|\boldsymbol{u}_{b}\right|$ accounts for bottom friction, where $\boldsymbol{u}_{b}=$ velocity evaluated at the 
seafloor, and $f=$ bottom friction coefficient, typically in the range of $10^{-3}-10^{-2}, v_{T}=$ constant eddy viscosity, $\nabla^{2}=\left(\partial^{2} / \partial x^{2}, \partial^{2} / \partial y^{2}\right), k_{1}=-0.127 h_{r}=$ evaluation level for the velocity $u_{1}$, $\zeta=-0.266 h_{r}=$ layer interface elevation, $s=$ evaluation level for the velocity $u_{2}$, and $\eta=$ free surface elevation.

\subsection{HD Serre's equations with improved dispersive performance}

To allow applications in a greater range of $h_{0} / \lambda$, other than shallow waters, a new set of extended Serre equations, with additional terms of dispersive origin, is developed and tested in [1] and [2] by comparisons with the available test data. The equations are solved using an efficient finite-difference method, whose consistency and stability are tested in that work by comparison with a closed-form solitary wave solution of the Serre equations.

From the equation system (20), by adding and subtracting terms of dispersive origin, using the approximation $u_{t}=-g \eta_{x}$ and considering the parameters $\alpha, \beta$ and $\gamma$, with $\beta=1.5 \alpha-0.5 \gamma$, allows to obtain a new system of equations with improved linear dispersion characteristics:

$$
\begin{aligned}
& h_{t}+(u h)_{x}=0 \\
& u_{t}+u u_{x}+g(h+\xi)_{x}+(1+\alpha)\left(\Omega u_{t}-h h_{x} u_{x t}\right)-(1+\beta) \frac{h^{2}}{3} u_{x x t} \\
& \quad+\alpha g \Omega(h+\xi)_{x}-\alpha g h h_{x}(h+\xi)_{x x}-\beta g \frac{h^{2}}{3}(h+\xi)_{x x x}-h h_{x} u u_{x x} \\
& \quad+\frac{h^{2}}{3}\left(u_{x} u_{x x}-u u_{x x x}\right)+h\left(u_{x}\right)^{2}(h+\xi)_{x}+\xi_{x x} u^{2}(h+\xi)_{x} \\
& \quad+\left(\Omega+h \xi_{x x}\right) u u_{x}+\frac{h}{2} \xi_{x x x} u^{2}=0
\end{aligned}
$$

After linearization of the equation system (75), the dispersion relation (58) is obtained. As for the Boussinesq approach obtained by Liu and Sun, equating equation (58) with the linear dispersion relation $\omega^{2} / g k=\tanh \left(k h_{r}\right)$, using the approach (60), values of $\alpha=0.1308$ and $\gamma=-0.0076$ are obtained, so that $\beta=0.20$. It should be noted that the Serre's equation system (20) is recovered by setting $\alpha=\beta=0$.

\section{Numerical formulations and applications}

\subsection{HD Boussinesq-type approaches}

\subsubsection{WACUP numerical model}

An extension of the Boussinesq model (24) to take into account wave-current interactions has been derived and presented in [5]. This model is named WACUP (a 2HD WAve Plus CUrrent 
Boussinesq-type model). With dimensional variables, taking mean quantities of the horizontal velocity components $U=\left(u+u_{c}\right)$ and $V=\left(v+v_{c}\right)$, where index $c$ represents current and $u=U-u_{c}$ and $v=V-v_{c}$, the final set of these equations are written as follows:

$$
\begin{gathered}
h_{t}+(h U)_{x}+(h V)_{y}=0 \\
U_{t}+U U_{x}+V U_{y}+g(h+\xi)_{x}-\frac{h_{r}^{2}}{3}\left(U_{x x t}+V_{x y t}\right) \\
-\frac{h_{r}^{2}}{3}\left[u_{c}\left(U_{x x}+V_{x y}\right)+v_{c}\left(U_{x y}+V_{y y}\right)\right]_{x}+h_{r}\left(\frac{1}{2} \xi_{t t}+u_{c} \xi_{x t}+v_{c} \xi_{y t}\right)_{x} \\
-v_{l}\left(U_{x x}+U_{y y}\right)-\frac{\tau_{s_{x}}}{\rho h}+\frac{\tau_{b_{x}}}{\rho h}=0 \\
V_{t}+U V_{x}+V V_{y}+g(h+\xi)_{y}-\frac{h_{r}^{2}}{3}\left(U_{x y t}+V_{y y t}\right) \\
-\frac{h_{r}^{2}}{3}\left[u_{c}\left(U_{x x}+V_{x y}\right)+v_{c}\left(U_{x y}+V_{y y}\right)\right]_{y}+h_{r}\left(\frac{1}{2} \xi_{t t}+u_{c} \xi_{x t}+v_{c} \xi_{y t}\right)_{y} \\
-v_{l}\left(V_{x x}+V_{y y}\right)-\frac{\tau_{s_{y}}}{\rho h}+\frac{\tau_{b_{y}}}{\rho h}=0
\end{gathered}
$$

where $\tau_{s}$ and $\tau_{b}$ represent stresses on surface and at bottom, respectively.

The standard Boussinesq model (24) and the extended system of equations (76)-(78) are solved in [8] and [5], respectively, using an efficient finite element method for spatial discretization of the partial differential equations. Firstly, the $(U, V)$ derivatives in time and third spatial derivatives are grouped in two equations. This means that an equivalent system of five equations is solved instead of the original equation system (76)-(78). The final equation system takes the following form:

$$
\begin{gathered}
h_{t}+h U_{x}+U h_{x}+h V_{y}+V h_{y}=0 \\
r_{t}+u_{c} r_{x}+v_{c} r_{y}=-u U_{x}-v U_{y}-g(h+\xi)_{x} \\
+\frac{h_{r}^{2}}{3}\left[\left(u_{c}\right)_{x}\left(U_{x x}+U_{y y}\right)+\left(v_{c}\right)_{x}\left(V_{x x}+V_{y y}\right)\right]-h_{r}\left(\frac{1}{2} \xi_{t t}+u_{c} \xi_{x t}+v_{c} \xi_{y t}\right)_{x} \\
+\left[v_{l}+\frac{2}{3} h_{r}\left(\xi_{t}+u_{c} \xi_{x}+v_{c} \xi_{y}\right)\right]\left(U_{x x}+U_{y y}\right)+\frac{\tau_{s_{x}}}{\rho h}-\frac{\tau_{b_{x}}}{\rho h}=0
\end{gathered}
$$




$$
\begin{gathered}
s_{t}+u_{c} s_{x}+v_{c} s_{y}=-u V_{x}-v V_{y}-g(h+\xi)_{y} \\
+\frac{h_{r}^{2}}{3}\left[\left(u_{c}\right)_{y}\left(U_{x x}+U_{y y}\right)+\left(v_{c}\right)_{y}\left(V_{x x}+V_{y y}\right)\right]-h_{r}\left(\frac{1}{2} \xi_{t t}+u_{c} \xi_{x t}+v_{c} \xi_{y t}\right)_{\mathrm{y}} \\
+\left[v_{l}+\frac{2}{3} h_{r}\left(\xi_{t}+u_{c} \xi_{x}+v_{c} \xi_{y}\right)\right]\left(V_{x x}+V_{y y}\right)+\frac{\tau_{s_{y}}}{\rho h}-\frac{\tau_{b_{y}}}{\rho h}=0 \\
U-\frac{h_{r}^{2}}{3}\left(U_{x x}+U_{y y}\right)=r \\
V-\frac{h_{r}^{2}}{3}\left(V_{x x}+V_{y y}\right)=s
\end{gathered}
$$

It should be noted that weakly vertical rotational flows were assumed, which strictly correspond to a limitation of the numerical method.

As the values of variables $h, U, V, r$ and $s$ are known at time $t$, we can use a numerical procedure based on the following steps to compute the corresponding values at time $t+\Delta t$ (for details see [5]):

1. The equation (79) allows us to predict the values of variable $h\left(h_{p}^{t+\Delta t}\right)$, considering the known values of $\mathrm{h}, U$ and $V$ at time $t$ in the whole domain.

2. Equations (80) and (81) make it possible to predict the values of variables $r\left(r_{p}^{t+\Delta t}\right)$ and $s$ $\left(s_{p}^{t+\Delta t}\right)$, taking into account the values of $U^{t}, V^{t}, r^{t}, s^{t}$ and $\widetilde{h}^{t+\Delta t}=0.5 h^{t}+0.5 h_{p}^{t+\Delta t}$ known for the whole domain.

3. Solutions of equations (82) and (83) give us the values of the mean-averaged velocity components $U$ and $V\left(U^{t+\Delta t}\right.$ and $\left.V^{t+\Delta t}\right)$, taking into account the predicted values of $r$ and $s\left(r_{p}^{t+\Delta t}\right.$ and $s_{p}^{t+\Delta t}$, respectively).

4. Equation (79) allows us to compute the depth $h$ at time $t+\Delta t$ (values of $h^{t+\Delta t}$ ) considering the values of variables $h^{t}, U^{t+05 \Delta t}=0.5 U^{t}+0.5 U^{t+\Delta t}$ and $V^{t+05 \Delta t}=0.5 V^{t}+0.5 V^{t+\Delta t}$ known for the whole domain.

5. Equations (80) and (81) allow us to compute the values of variables $r$ and $s$ at time $t+\Delta t$ (values of $r^{t+\Delta t}$ and $s^{t+\Delta t}$ ), taking into account the values of $r^{t}, s^{t} h^{t+05 \Delta t}=0.5 h^{t}+0.5 h^{t+\Delta t}$, $U^{t+05 \Delta t}=0.5 U^{t}+0.5 U^{t+\Delta t}$ and $V^{t+05 \Delta t}=0.5 V^{t}+0.5 V^{t+\Delta t}$ known for the whole domain.

The Petrov-Galerkin procedure is utilized to achieve solutions for the unknowns $h, r$ and $s$. According to the weighted residual technique, minimization requires the "orthogonality" of the residual $R_{J}$ to a set of weighting functions $W_{i, J}$, i.e., 


$$
\int_{\Delta^{e}} W_{i, J} R_{J} d \Delta^{e}=0, i=1, \cdots, n
$$

where the general form of the weighting functions applied to these equations is defined as:

$$
W_{i, J}=N_{i}+\delta_{u i}\left(N_{x}\right)_{i}+\delta_{v i}\left(N_{y}\right)_{i}, i=1, \cdots, n
$$

and where the coefficients $\delta_{u_{i}}$, and $\delta_{v_{i}}$ are functions of: (i) the local velocities $U$ and $V$; (ii) the ratio of the wave amplitude to the water depth, and (iii) the element length. To illustrate this procedure, a complete solution of equations (79) (80) and (82) is presented here in detail. Introducing in equation (79), the approximated values are given by:

$$
p \approx \hat{p}=\sum_{1=1}^{n} N_{i} p_{i}
$$

the following residual $R_{79}$ is obtained:

$$
R_{79}=\hat{h}_{t}+\hat{h} \hat{U}_{x}+\hat{U} \hat{h}_{x}+\hat{h} \hat{V}_{y}+\hat{V} \hat{h}_{y}
$$

The $R_{79}$ error minimization leads to the following equation:

$$
\begin{aligned}
& \int_{\Delta^{e}} W_{i, 79} R_{79} d \Delta^{e}= \int_{\Delta^{e}} W_{i, 79}\left(\hat{h}_{t}+\hat{h} \hat{U}_{x}+\hat{U} \hat{h}_{x}+\hat{h} \hat{V}_{y}+\hat{V} \hat{h}_{y}\right) d \Delta^{e} \\
&=\int_{\Delta^{e}} W_{i, 79}\left[\sum_{j=1}^{n} N_{j}\left(h_{t}\right)_{j}+\sum_{k=1}^{n}\left(N_{x}\right)_{k} U_{k} \sum_{j=1}^{n} N_{j} h_{j}+\sum_{k=1}^{n} N_{k} U_{k} \sum_{j=1}^{n}\left(N_{x}\right)_{j} h_{j}\right. \\
&\left.+\sum_{k=1}^{n}\left(N_{y}\right)_{k} V_{k} \sum_{j=1}^{n} N_{j} h_{j}+\sum_{k=1}^{n} N_{k} V_{k} \sum_{j=1}^{n}\left(N_{y}\right)_{j} h_{j}\right] d \Delta^{e}=0
\end{aligned}
$$

In the matrix form, this equation may be written as:

$$
A h_{t}+B h=0
$$

where 


$$
\begin{aligned}
a_{i, j}= & \int_{\Delta^{e}} W_{i, 79} N_{j} d \Delta^{e} ; i, j=1, \cdots, n \\
b_{i, j}= & \int_{\Delta^{e}} W_{i, 79} \sum_{k=1}^{n} N_{k} U_{k}\left(N_{x}\right)_{j} d \Delta^{e}+\int_{\Delta^{e}} W_{i, 79} \sum_{k=1}^{n}\left(N_{x}\right)_{k} U_{k} N_{j} d \Delta^{e} \\
& +\int_{\Delta^{e}} W_{i, 79} \sum_{k=1}^{n}\left(N_{y}\right)_{k} V_{k} N_{j} d \Delta^{e}+\int_{\Delta^{e}} W_{i, 79} \sum_{k=1}^{n} N_{k} V_{k}\left(N_{y}\right)_{j} d \Delta^{e} ; i, j=1, \cdots, n
\end{aligned}
$$

The residual $R_{80}$ is written as:

$$
\begin{aligned}
R_{80}= & \hat{r}_{t}+\hat{u}_{c} \hat{r}_{x}+\hat{v}_{c} \hat{r}_{y}+\hat{u} \hat{U}_{x}+\hat{v} \hat{U}_{y} \\
& +g(\hat{h}+\hat{\xi})_{x}-\frac{\hat{h}_{r}^{2}}{3}\left[\left(\hat{u}_{c}\right)_{x}\left(\hat{U}_{x x}+\hat{U}_{y y}\right)+\left(\hat{v}_{c}\right)_{x}\left(\hat{V}_{x x}+\hat{V}_{y y}\right)\right] \\
& +\hat{h}_{r}\left[\frac{1}{2}\left(\hat{\xi}_{t t}\right)_{x}+\left(\hat{u}_{c}\right)_{x}\left(\hat{\xi}_{t}\right)_{x}+\hat{u}_{c}\left(\hat{\xi}_{t}\right)_{x x}+\left(\hat{v}_{c}\right)_{x}\left(\hat{\xi}_{t}\right)_{y}+\hat{v}_{c}\left(\hat{\xi}_{t}\right)_{x y}\right] \\
& -v_{l}\left(\hat{U}_{x x}+\hat{U}_{y y}\right)-\frac{\tau_{s_{x}}}{\rho h}+\frac{\tau_{b_{x}}}{\rho h}
\end{aligned}
$$

It should be noted that the term $\frac{2}{3} h_{r}\left(\xi_{t}+u_{c} \xi_{x}+v_{c} \xi_{y}\right)\left(V_{x x}+V_{y y}\right)$ of equation (80) is of order $\sigma^{4}$ or greater. For this reason, it is not considerd in the numerical developments. Similarly, and for the same reason, considering $f=h_{r}^{2} / 3$, all terms involving $\nabla f$ in the numerical developments are omitted.

The Green's theorem is used to solve the second derivatives present in equation (80) (residual $\left.R_{80}\right)$, and in equations (81) to (83), i.e., considering $\hat{p}=(\hat{U}, \hat{V})$, from $\hat{p}_{x x}+\hat{p}_{y y}$ we obtain:

$$
\int_{\Delta^{e}} W_{i, l}\left(\hat{p}_{x x}+\hat{p}_{y y}\right) d \Delta^{e}=\int_{\Delta^{e}} W_{i, l} \sum_{\mathrm{j}=1}^{\mathrm{n}}\left[\left(N_{x x}\right)_{j}+\left(N_{y y}\right)_{j}\right] p_{j} d \Delta^{e}
$$

and so

$$
\begin{aligned}
\int_{\Delta^{e}} W_{i, l} \sum_{\mathrm{j}=1}^{\mathrm{n}}\left[\left(N_{x x}\right)_{j}+\left(N_{y y}\right)_{j}\right] p_{j} d \Delta^{e}=-\int_{\Delta^{e}}\left(W_{x}\right)_{i, l} \sum_{j=1}^{n}\left(N_{x}\right)_{j} p_{j} d \Delta^{e} \\
-\int_{\Delta^{e}}\left(W_{y}\right)_{i, l} \sum_{j=1}^{n}\left(N_{y}\right)_{j} p_{j} d \Delta^{e}+\oint_{\Gamma^{e}} W_{i, l} \sum_{j=1}^{n}\left(N_{n}\right)_{j} p_{j} d \Gamma^{e} ; p=(U, V)
\end{aligned}
$$

Retaining terms up to order $\sigma^{3}$, the $R_{80}$ error minimization leads to: 


$$
\begin{aligned}
& \int_{\Delta^{e}} W_{i, 80} R_{80} d \Delta^{e}=\int_{\Delta^{e}} \int_{i, 80}\left[\sum_{j=1}^{n} N_{j}\left(r_{t}\right)_{j}+\sum_{k=1}^{n} N_{k}\left(u_{c}\right)_{k} \sum_{j=1}^{n}\left(N_{x}\right)_{j} r_{j}\right. \\
& +\sum_{k=1}^{n} N_{k}\left(v_{c}\right)_{k_{x}} \sum_{j=1}^{n}\left(N_{y}\right)_{j} r_{j}+\sum_{k=1}^{n} N_{k} u_{k} \sum_{j=1}^{n}\left(N_{x}\right)_{j} U_{j}+\sum_{k=1}^{n} N_{k} v_{k} \sum_{j=1}^{n}\left(N_{y}\right)_{j} U_{j} \\
& \left.+g \sum_{j=1}^{n}\left(N_{x}\right)_{j}(h+\xi)_{j}\right]-\sum_{l=1}^{n} N_{l} \frac{\left(h_{r}^{2}\right)_{l}}{3} \sum_{k=1}^{n}\left(N_{x}\right)_{k}\left[\left(u_{c}\right)_{k} U_{x^{2} y^{2}}+\left(v_{c}\right)_{k} V_{x^{2} y^{2}}\right] \\
& +W_{i, 80}\left[\sum_{k=1}^{n} N_{k} \frac{\left(h_{r}\right)_{k}}{2} \sum_{j=1}^{n}\left(N_{x}\right)_{j}\left(\xi_{t t}\right)_{j}+\sum_{l=1}^{n} N_{l}\left(h_{r}\right)_{l} \sum_{k=1}^{n}\left(N_{x}\right)_{k}\left(u_{c}\right)_{k} \sum_{j=1}^{n}\left(N_{x}\right)_{j}\left(\xi_{t}\right)_{j}\right. \\
& \left.+\quad \sum_{l=1}^{n} N_{l}\left(h_{r}\right)_{l} \sum_{k=1}^{n}\left(N_{x}\right)_{k}\left(v_{c}\right)_{k} \sum_{j=1}^{n}\left(N_{y}\right)_{j}\left(\xi_{t}\right)_{j}\right] \\
& +\sum_{l=1}^{n} N_{l}\left(h_{r}\right)_{l}\left[\sum_{k=1}^{n} N_{k}\left(u_{c}\right)_{k}\left(\tilde{\xi}_{t}\right)_{x x}+\sum_{k=1}^{n} N_{k}\left(v_{c}\right)_{k}\left(\tilde{\xi}_{t}\right)_{x y}\right]-v_{l} U_{x^{2} y^{2}} \\
& \left.-W_{i, 80} \sum_{k=1}^{n} N_{k}\left[\left(\tau_{s_{x}}\right)_{k}-\left(\tau_{b_{x}}\right)_{k}\right] / \sum_{j=1 h}^{n} N_{j} h_{j}\right\} d \Delta^{e}=0
\end{aligned}
$$

where

$$
\left(\tilde{\xi}_{t}\right)_{x x}=-\left(W_{x}\right)_{i, 80} \sum_{j=1}^{n}\left(N_{x}\right)_{j}\left(\xi_{t}\right)_{j^{\prime}}\left(\tilde{\xi}_{t}\right)_{x y}=-\left(W_{x}\right)_{i, 80} \sum_{j=1}^{n}\left(N_{y}\right)_{j}\left(\xi_{t}\right)_{j}
$$

and

$$
p_{x^{2} y^{2}}=-\left[\left(W_{x}\right)_{i, J} \sum_{j=1}^{n}\left(N_{x}\right)_{j}+\left(W_{y}\right)_{i, J} \sum_{j=1}^{n}\left(N_{y}\right)_{j}\right] p_{j}, p=(U, V)
$$

In the matrix form, this equation may be written as:

$$
A r_{t}+B r=C
$$

where

$$
\begin{gathered}
a_{i, j}=\int_{\Delta^{e}} W_{i, 80} N_{j} d \Delta^{e}, i, j=1, \cdots, n \\
b_{i, j}=\int_{\Delta^{e}} W_{i, 80}\left[\sum_{k=1}^{n} N_{k}\left(u_{c}\right)_{k}\left(N_{x}\right)_{j}+\sum_{k=1}^{n} N_{k}\left(v_{c}\right)_{k}\left(N_{y}\right)_{j}\right] d \Delta^{e}, i, j=1, \cdots, n
\end{gathered}
$$




$$
\begin{aligned}
c_{i}= & -\int_{\Delta^{e}}\left\{W _ { i , 8 0 } \left[\sum_{k=1}^{n} N_{k} u_{k} \sum_{j=1}^{n}\left(N_{x}\right)_{j} U_{j}+\sum_{k=1}^{n} N_{k} v_{k} \sum_{j=1}^{n}\left(N_{y}\right)_{j} U_{j}\right.\right. \\
& \left.+g \sum_{j=1}^{n}\left(N_{x}\right)_{j}(h+\xi)_{j}\right]-\sum_{l=1}^{n} N_{l} \frac{\left(h_{r}^{2}\right)_{l}}{3} \sum_{k=1}^{n}\left(N_{x}\right)_{k}\left[\left(u_{c}\right)_{k} U_{x^{2} y^{2}}+\left(v_{c}\right)_{k} V_{x^{2} y^{2}}\right] \\
& +W_{i, 80}\left[\sum_{k=1}^{n} N_{k} \frac{\left(h_{r}\right)_{k}}{2} \sum_{j=1}^{n}\left(N_{x}\right)_{j}\left(\xi_{t t}\right)_{j}+\sum_{l=1}^{n} N_{l}\left(h_{r}\right)_{l} \sum_{k=1}^{n}\left(N_{x}\right)_{k}\left(u_{c}\right)_{k} \sum_{j=1}^{n}\left(N_{x}\right)_{j}\left(\xi_{t}\right)_{j}\right. \\
& \left.+\sum_{l=1}^{n} N_{l}\left(h_{r}\right)_{l} \sum_{k=1}^{n}\left(N_{x}\right)_{k}\left(v_{c}\right)_{k} \sum_{j=1}^{n}\left(N_{y}\right)_{j}\left(\xi_{t}\right)_{j}\right] \\
& +\sum_{l=1}^{n} N_{l}\left(h_{r}\right)_{l}\left[\sum_{k=1}^{n} N_{k}\left(u_{c}\right)_{k}\left(\tilde{\xi}_{t}\right)_{x x}+\sum_{k=1}^{n} N_{k}\left(v_{c}\right)_{k}\left(\xi_{t}\right)_{x y}\right]-v_{l} U_{x^{2} y^{2}} \\
& \left.-W_{i, 80} \sum_{k=1}^{n} N_{k}\left[\left(\tau_{s_{x}}\right)_{k}-\left(\tau_{b_{x}}\right)_{k}\right] / \sum_{j=1 h}^{n} N_{j} h_{j}\right\} d \Delta^{e}=0, i=1, \ldots . n
\end{aligned}
$$

The residual $R_{82}$ is written as:

$$
R_{82}=\hat{U}-\frac{\hat{h}_{r}^{2}}{3}\left(\frac{\partial^{2} \hat{U}}{\partial x^{2}}+\frac{\partial^{2} \hat{U}}{\partial y^{2}}\right)-\hat{r}
$$

According to Galerkin's procedure, after using integration by parts (or Green's theorem) to reduce the second derivatives, the $R_{82}$ error minimization leads to the following equation:

$$
\begin{aligned}
\int_{\Delta^{e}} W_{i, 82} R_{82} d \Delta^{e} & =\int_{\Delta^{e}}\left\{W_{i, 82} \sum_{j=1}^{n} N_{j} U_{j}\right. \\
& +\sum_{k=1}^{n} N_{k} \frac{\left(h_{r}^{2}\right)_{k}}{3}\left[\left(W_{x}\right)_{i, 82} \sum_{j=1}^{n}\left(N_{x}\right)_{j}+\left(W_{y}\right)_{i, 82} \sum_{j=1}^{n}\left(N_{y}\right)_{j}\right] U_{j} \\
& \left.-W_{i, 82} \sum_{j=1}^{n} N_{j} r_{j}\right\} d \Delta^{e}-\oint_{\Gamma^{e}} N_{k} \frac{\left(h_{r}^{2}\right)_{k}}{3} W_{i, 82}\left(N_{n}\right)_{j} U_{j} d \Gamma^{e}
\end{aligned}
$$

The last term of (102) can be written as:

$$
\begin{aligned}
\oint_{\Gamma^{e}} N_{k} \frac{\left(h_{r}^{2}\right)_{k}}{3} W_{i, 82}\left(N_{n}\right)_{j} U_{j} d \Gamma^{e} & =\oint_{\Gamma_{i}^{e}} N_{p} \frac{\left(h_{r}^{2}\right)_{p}}{3} W_{q, 82}\left(N_{n}\right)_{r} U_{r} d \Gamma_{i}^{e} \\
& +\oint_{\Gamma_{e}^{e}} N_{p} \frac{\left(h_{r}^{2}\right)_{p}}{3} W_{q, 82} U_{n} d \Gamma_{e}^{e}
\end{aligned}
$$


where $p, q=1, \cdots, n^{e}, n^{e}$ being the number of nodes in the corresponding element side coincident with the boundary domain, $\Gamma_{i}^{e}$ represents the element sides within the domain, with the corresponding integral null because the resulting element contributions are equal, but with opposite signals, and $\Gamma_{e}^{e}$ represents the element sides coincident with the boundary domain. Accordingly, an equivalent form of equation (102) may be written as follows:

$$
\begin{aligned}
\int_{\Delta^{e}} W_{i, 82} R_{82} d \Delta^{e}=\int_{\Delta^{e}}\left\{W_{i, 82} \sum_{j=1}^{n} N_{j}\right. \\
\left.\quad+\sum_{k=1}^{n} N_{k} \frac{h\left(\begin{array}{l}
2 \\
r
\end{array}\right) k}{3}\left[\left(W_{x}\right)_{i, 82} \sum_{j=1}^{n}\left(N_{x}\right)_{j}+\left(W_{y}\right)_{i, 82} \sum_{j=1}^{n}\left(N_{y}\right)_{j}\right]\right\} U_{j} d \Delta^{e} \\
\quad=\int_{\Delta^{e}} W_{i, 82} \sum_{j=1}^{n} N_{j} r_{j} d \Delta^{e}+\oint_{\Gamma^{e}} N_{p} \frac{\left(h_{r}^{2}\right)_{p}}{3} W_{q, 82} U_{n} d \Gamma_{e}^{e} \\
p, q=1, \cdots, n^{e}
\end{aligned}
$$

In the matrix form, this equation may be written as:

$$
A U=B
$$

where

$$
\begin{gathered}
a_{i, j}=\int_{\Delta^{e}}\left\{W_{i, 82} \sum_{j=1}^{n} N_{j}+\sum_{k=1}^{n} N_{k} \frac{\left(h_{r}^{2}\right)_{k}}{3}\left[\left(W_{x}\right)_{i, 82} \sum_{j=1}^{n}\left(N_{x}\right)_{j}+\left(W_{y}\right)_{i, 82} \sum_{j=1}^{n}\left(N_{y}\right)_{j}\right]\right\} d \Delta^{e} \\
b_{i}=\int_{\Delta^{e}} W_{i, 82} \sum_{j=1}^{n} N_{j} r_{j} d \Delta^{e}+\oint_{\Gamma^{e}} N_{p} \frac{\left(h_{r}^{2}\right)_{p}}{3} W_{q, 82} U_{n} d \Gamma^{e} \\
p, q=1, \cdots, n^{e} .
\end{gathered}
$$

As recommended in [5] "a suitable grid is normally crucial to the success of a finite element model. In our case, the following rules must be fulfilled for its generation.

a. Element side lower than the local depth.

b. Minimum of 20 to 25 elements per wave length.

c. Courant number always lower than one in the whole domain".

\subsubsection{Real case study using the WACUP model}

The fortification of S. Lourenço da Cabeça Seca (lighthouse of Bugio) - Tagus estuary (Portugal) - has endured over the course of four centuries the continuous action of waves and currents, 
as well as bathymetric modifications resulting from the movement of significant quantities of sand in the area where it is located.

With the intention of preventing the destruction of this fortification, several studies were conducted to evaluate the best protection structure. The studies have led to a protective structure which consists of a circular dissipation platform, with a level of $2 \mathrm{~m}$ (HZ) and about $80 \mathrm{~m}$ radius (Figure 1 ).

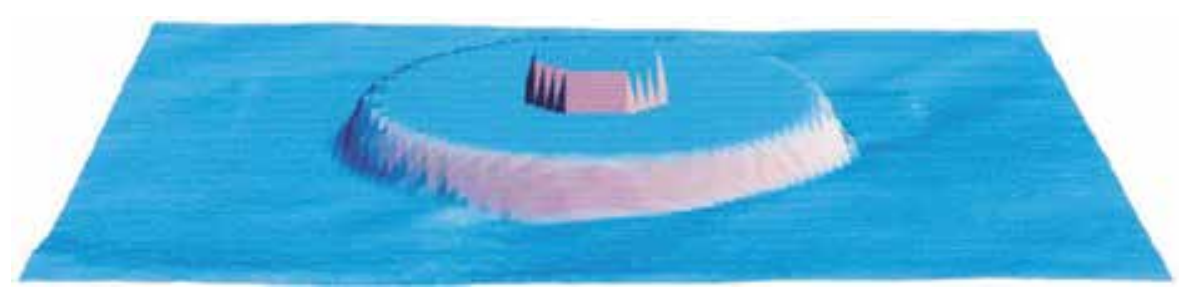

Figure 1. Submerged dissipation platform to protect the Bugio lighthouse, situated at the mouth of the Tagus estuary.

The wave-current Boussinesq-type model WACUP was used to obtain the time-dependent hydrodynamic characteristics of the joint action of a relatively common wave over the high flow tide current. The wave characteristics are: wave height, $H=3.5 \mathrm{~m}$, period $T=12 \mathrm{~s}$ and direction $=180^{\circ}$. Figure 2 shows the free surface level when the wave approaches the platform and its action on the Bugio lighthouse. Figure 3 shows a free surface water level, in a quasistationary state.

As can be seen, the wave action on the fortification was drastically reduced as a consequence of the wave breaking, refraction and diffraction on the dissipation platform constructed around the fortification.

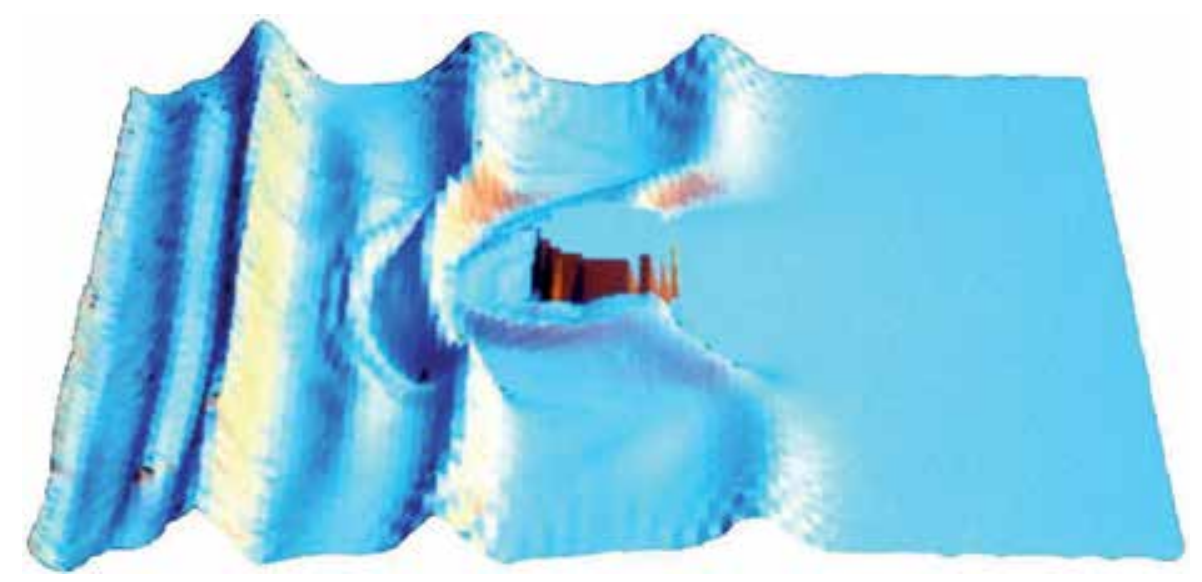

Figure 2. Perspective of the free surface obtained by simulation around the Bugio lighthouse, situated in the Tagus estuary, Portugal, in a transient condition. 


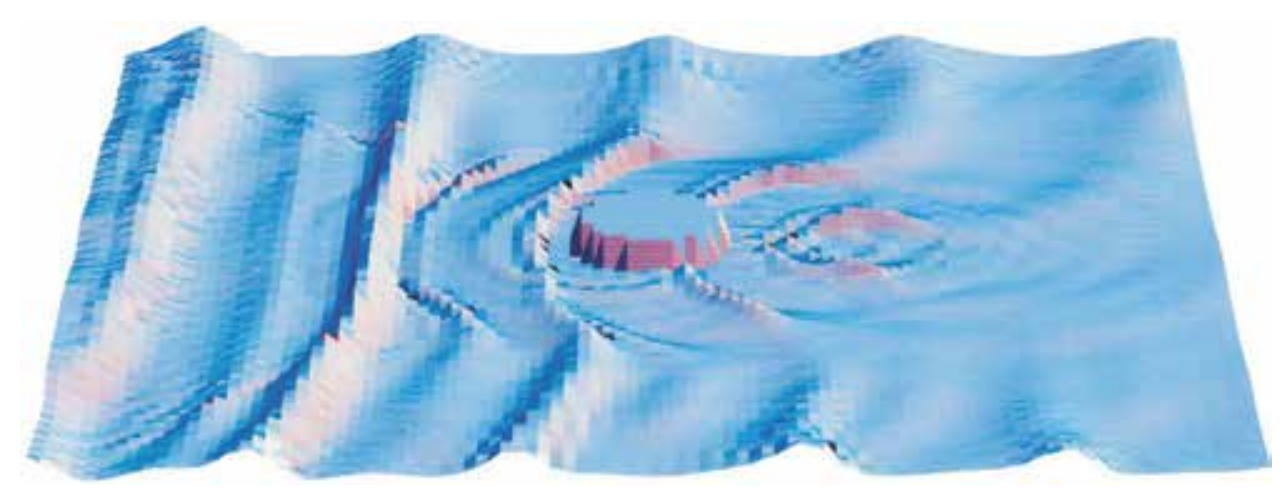

Figure 3. Protection of the Bugio lighthouse situated at the mouth of the Tagus estuary. Perspective of the free surface obtained with a numerical simulation, in a quasi-steady state.

\subsubsection{COULWAVE one-layer model}

A numerical scheme similar to that of [38] and [39] is utilized by [25], with the inclusion of extra nonlinear terms. Basically using the same high-order predictor-corrector scheme, [25] developed a numerical code (COULWAVE) based on Nwogu's equations for one and two layers. Parameterizations of bottom friction and wave breaking have been included in the code, as well as a moving boundary scheme to simulate wave runup and rundown. A finitedifference algorithm is used for the general one- and two-layer model equations.

According to [9], "the equations are solved using a high-order predictor-corrector scheme, employing a third order in time explicit Adams-Bashforth predictor step, and a fourth order in time Adams-Moulton implicit corrector step" [31]. Also in accordance with [9], "the implicit corrector step must be iterated until a convergence criterion is satisfied".

In order to solve numerically the nondimensional equations (69)-(71), these are previously rewritten in dimensional variables. Then, to simplify the predictor-corrector equations, the velocity time derivatives in the momentum equations are grouped into the dimensional form (for details see [25]):

$$
\begin{aligned}
& U=u+\frac{k^{2}-\eta^{2}}{2} u_{x x}+(k-\eta)\left(h_{r} u\right)_{x x}-\eta_{x}\left[\eta u_{x}+\left(h_{r} u\right)_{x}\right] \\
& V=v+\frac{k^{2}-\eta^{2}}{2} v_{y y}+(k-\eta)\left(h_{r} v\right)_{y y}-\eta_{y}\left[\eta v_{y}+\left(h_{r} v\right)_{y}\right]
\end{aligned}
$$

where subscripts denote partial derivatives. For reasons of stability and less iterations required in the process of convergence, the nonlinear time derivatives, arisen from the nonlinear dispersion terms $\nabla\left[\eta\left(\nabla .\left(h_{r} u_{\alpha}\right)_{t}+h_{r_{t t}} / \varepsilon\right)\right]$ and $\nabla\left[\left(\eta^{2} / 2\right) \nabla . u_{\alpha t}\right]$, can be reformulated using the relations: 


$$
\begin{gathered}
\nabla\left[\eta\left(\nabla \cdot\left(h_{r} u_{\alpha}\right)_{t}+\frac{h_{r t t}}{\varepsilon}\right)\right]=\nabla\left[\eta\left(\nabla \cdot\left(h_{r} u_{\alpha}\right)+\frac{h_{r t}}{\varepsilon}\right)\right]_{t}-\nabla\left[\eta_{t}\left(\nabla \cdot\left(h_{r} u_{\alpha}\right)+\frac{h_{r t}}{\varepsilon}\right)\right] \\
\nabla\left(\frac{\eta^{2}}{2} \nabla \cdot u_{\alpha t}\right)=\nabla\left(\frac{\eta^{2}}{2} \nabla \cdot u_{\alpha}\right)_{t}-\nabla\left(\eta \eta_{t} \nabla \cdot u_{\alpha}\right)
\end{gathered}
$$

The explicit predictor equations are:

$$
\begin{gathered}
\eta_{i, j}^{n+1}=\eta_{i, j}^{n}+\frac{\Delta t}{12}\left(23 E_{i, j}^{n}-16 E_{i, j}^{n-1}+5 E_{i, j}^{n-2}\right) \\
U_{i, j}^{n+1}=U_{i, j}^{n}+\frac{\Delta t}{12}\left(23 F_{i, j}^{n}-16 F_{i, j}^{n-1}+5 F_{i, j}^{n-2}\right)+2\left(F_{1}\right)_{i, j}^{n}-3\left(F_{1}\right)_{i, j}^{n-1}+\left(F_{1}\right)_{i, j}^{n-2} \\
V_{i, j}^{n+1}=V_{i, j}^{n}+\frac{\Delta t}{12}\left(23 G_{i, j}^{n}-16 G_{i, j}^{n-1}+5 G_{i, j}^{n-2}\right)+2\left(G_{1}\right)_{i, j}^{n}-3\left(G_{1}\right)_{i, j}^{n-1}+\left(G_{1}\right)_{i, j}^{n-2}
\end{gathered}
$$

where

$$
\begin{gathered}
E=-h_{r t}-\left[\left(\eta+h_{r}\right) u\right]_{x}-\left[\left(\eta+h_{r}\right) v\right]_{y} \\
+\left\{\left(\eta+h_{r}\right)\left[\left(\frac{1}{6}\left(\eta^{2}-\eta h_{r}+h_{r}^{2}\right)-\frac{1}{2} k^{2}\right) S_{x}+\left(\frac{1}{2}\left(\eta-h_{r}\right)-k\right) T_{x}\right]\right\}_{x} \\
+\left\{\left(\eta+h_{r}\right)\left[\left(\frac{1}{6}\left(\eta^{2}-\eta h_{r}+h_{r}^{2}\right)-\frac{1}{2} k^{2}\right) S_{y}+\left(\frac{1}{2}\left(\eta-h_{r}\right)-k\right) T_{y}\right]\right\}_{y} \\
F=-\frac{1}{2}\left[\left(u^{2}\right)_{x}+\left(v^{2}\right)_{x}\right]-g \eta_{x}-k h_{r x t t}-k_{t} h_{r x t}+\left(E h_{r t}+\eta h_{r t t}\right)_{x} \\
-[E(\eta S+T)]_{x}-\left[\frac{1}{2}\left(k^{2}-\eta^{2}\right)\left(u S_{x}+v S_{y}\right)\right]_{x} \\
-\left[(k-\eta)\left(u T_{x}+v T_{y}\right)\right]_{x}-\frac{1}{2}\left[(T+\eta S)^{2}\right]_{x} \\
F_{1}=\frac{\eta^{2}-k^{2}}{2} v_{x y}-(k-\eta)\left(h_{r} v\right)_{x y}+\eta_{x}\left[\eta v_{y}+\left(h_{r} v\right)_{y}\right]
\end{gathered}
$$




$$
\begin{aligned}
G=-\frac{1}{2}[ & \left.\left(u^{2}\right)_{y}+\left(v^{2}\right)_{y}\right]-g \eta_{y}-k h_{r y t t}-k_{t} h_{r y t}+\left(E h_{r t}+\eta h_{r t t}\right)_{y} \\
& -[E(\eta S+T)]_{y}-\left[\frac{1}{2}\left(k^{2}-\eta^{2}\right)\left(u S_{x}+v S_{y}\right)\right]_{y} \\
& -\left[(k-\eta)\left(u T_{x}+v T_{y}\right)\right]_{y}-\frac{1}{2}\left[(T+\eta S)^{2}\right]_{y} \\
G_{1}= & \frac{\eta^{2}-k^{2}}{2} u_{x y}-(k-\eta)\left(h_{r} u\right)_{x y}+\eta_{y}\left[\eta u_{x}+\left(h_{r} u\right)_{x}\right]
\end{aligned}
$$

and

$$
S=u_{x}+v_{y} ; T=h_{r t}+\left(h_{r} u\right)_{x}+\left(h_{r} v\right)_{y}
$$

All first-order spatial derivatives are differenced with fourth-order $\left(\Delta x^{4}=\Delta y^{4}\right)$ accurate equations, which are five-point differences. Second-order spatial derivatives are approximated with three-point centered finite-difference equations, which are second-order accurate. The fourth-order implicit corrector expressions for the free surface elevation, $\eta$, and the horizontal velocities, $u$ and $v$, are:

$$
\begin{gathered}
\eta_{i, j}^{n+1}=\eta_{i, j}^{n}+\frac{\Delta t}{24}\left(9 E_{i, j}^{n+1}+19 E_{i, j}^{n}-5 E_{i, j}^{n-1}+E_{i, j}^{n-2}\right) \\
U_{i, j}^{n+1}=U_{i, j}^{n}+\frac{\Delta t}{24}\left(9 F_{i, j}^{n+1}+19 F_{i, j}^{n}-5 F_{i, j}^{n-1}+F_{i, j}^{n-2}\right)+\left(F_{1}\right)_{i, j}^{n+1}-\left(F_{1}\right)_{i, j}^{n} \\
V_{i, j}^{n+1}=V_{i, j}^{n}+\frac{\Delta t}{24}\left(9 G_{i, j}^{n+1}+19 G_{i, j}^{n}-5 G_{i, j}^{n-1}+G_{i, j}^{n-2}\right)+\left(G_{1}\right)_{i, j}^{n+1}-\left(G_{1}\right)_{i, j}^{n}
\end{gathered}
$$

As noted in [25], "the system is solved by first evaluating the predictor equations, then $u$ and $v$ are solved via (108) and (109), respectively. Both (108) and (109) yield a diagonal matrix after finite differencing. The matrices are diagonal, with a bandwidth of three (due to three-point finite differencing), and the efficient Thomas algorithm can be utilized. At this point in the numerical system, we have predictors for $\eta, u$ and $v$ ". Next, the corrector expressions are evaluated, and again $u$ and $v$ are determined from (108) and (109).

Also in accordance with [25], "the error is calculated, in order to determine if the implicit correctors need to be reiterated. The error criteria employed is a dual calculation, and requires that either" 


$$
\max \left|\frac{w^{n+1}-w_{*}^{n+1}}{w^{n+1}}\right|<\frac{\varepsilon}{100} \text { or } \frac{\sum\left|w^{n+1}-w_{*}^{n+1}\right|}{\sum\left|w^{n+1}\right|}<\varepsilon
$$

In these expressions, $w$ represents any of the variables $\eta, u$ and $v$, and $w_{*}$ is the previous iterations value. The value of the error is set to $10^{-6}$. Linear stability analysis performed by [38, 18] and [40] show that $\Delta t<\Delta x /(2 c)$ to ensure stability, where $c$ is the celerity.

\subsubsection{Research study using COULWAVE model}

For the analysis concerning coastal protection, the mean currents around a submerged structure (artificial reef) are analysed. The output of the COULWAVE model corresponds to the velocity values at a depth $0.531 h_{r}$ below the water surface. The velocity at this depth is used to determine the velocity cells near the shoreline that could give an indication of the sediment transport. Divergent cells indicate erosion near by the shoreline and convergent cells indicate sedimentation.

The numerical simulations to study the 2HD behavior of the hydrodynamics around the reef have been done for four cases: two reef geometries, varying the reef angle $\left(45^{\circ}\right.$ and $\left.66^{\circ}\right)$, and two wave conditions. The characteristics of the simulations for the different cases are described in Table 1.

\begin{tabular}{ccccccc}
\hline & $\begin{array}{c}\text { Reef angle } \\
\left({ }^{\circ}\right)\end{array}$ & $\begin{array}{c}\mathbf{H} \\
(\mathbf{m})\end{array}$ & $\begin{array}{c}\mathbf{T} \\
(\mathbf{s})\end{array}$ & $\begin{array}{c}\text { Number of grid points per } \\
\text { wavelength }\end{array}$ & Grid size (m) & Time step (s) \\
\hline $\mathrm{C} 1$ & 45 & 4.0 & 15 & 60 & 2.77 & 0.11999 \\
$\mathrm{C} 2$ & 66 & 4.0 & 15 & 70 & 2.37 & 0.10285 \\
$\mathrm{C} 3$ & 45 & 1.5 & 9 & 43 & 2.14 & 0.09288 \\
$\mathrm{C} 4$ & 66 & 1.5 & 9 & 43 & 2.14 & 0.09288 \\
\hline
\end{tabular}

Table 1. Main characteristics of the simulations performed.

As recorded in [28], "the computational domain is around $1870 \mathrm{~m}$ in the long-shore and $1670 \mathrm{~m}$ in the cross-shore direction with a constant node spacing of around $\Delta x=\Delta y=2.0 \mathrm{~m}$ and $a$ Courant number of 0.5. The total simulation time was $800 \mathrm{~s}$. A flat bottom is placed in front of the slope where waves are generated using the source function method ([39] Wei et al., 1995). The source function is located at $x=80$ m along the $y$ direction" (Figure 4 ).

Two sponge layers are used, one in front of the offshore boundary to absorb the outgoing wave energy, and the other on the beach, both with a width of 0.5 times the wavelength of the incident wave. The numerical results obtained by the model are the time series of the free surface elevation, the two velocity components, $u$ and $v$, and the wave breaking areas (Figure 5). 


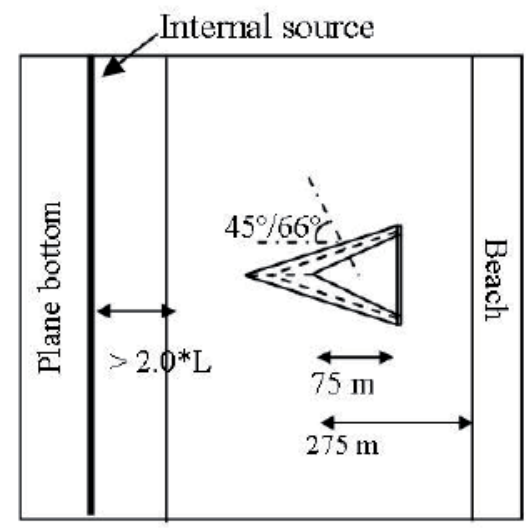

Figure 4. Schematic representation of the simulated geometry (not at scale) [28].

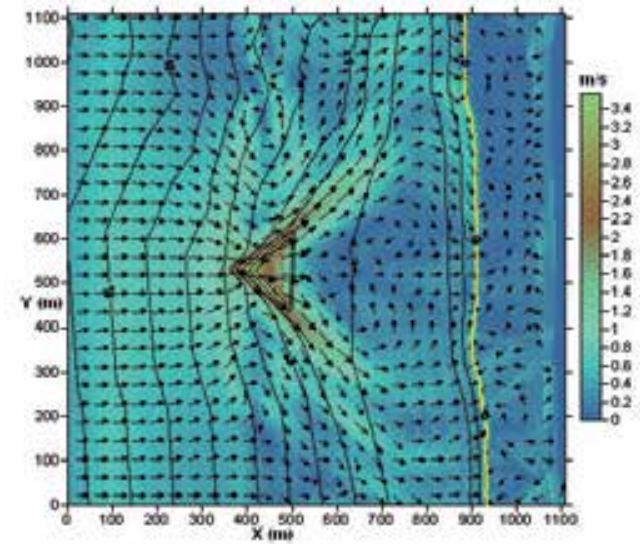

$\mathrm{C} 1: \mathrm{H}=4.0 \mathrm{~m}, \mathrm{~T}=15 \mathrm{~s}, \mathbf{4 5}^{\circ}$

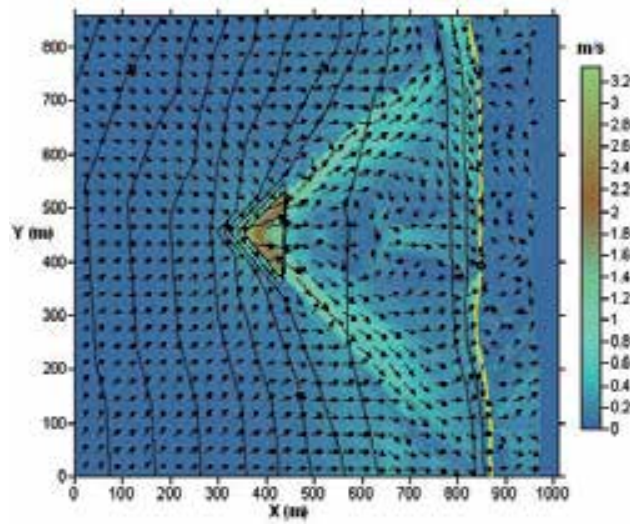

C3: $\mathrm{H}=1.5 \mathrm{~m}, \mathrm{~T}=9 \mathrm{~s}, 45^{\circ}$

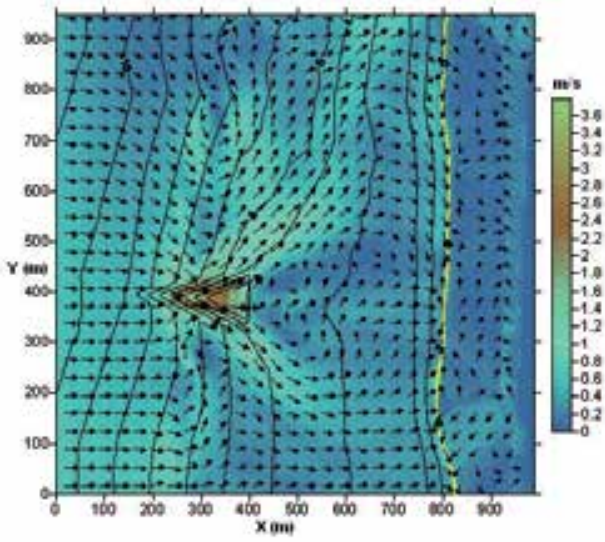

$\mathrm{C2}: \mathrm{H}=4.0 \mathrm{~m}, \mathrm{~T}=15 \mathrm{~s}, 66^{\circ}$

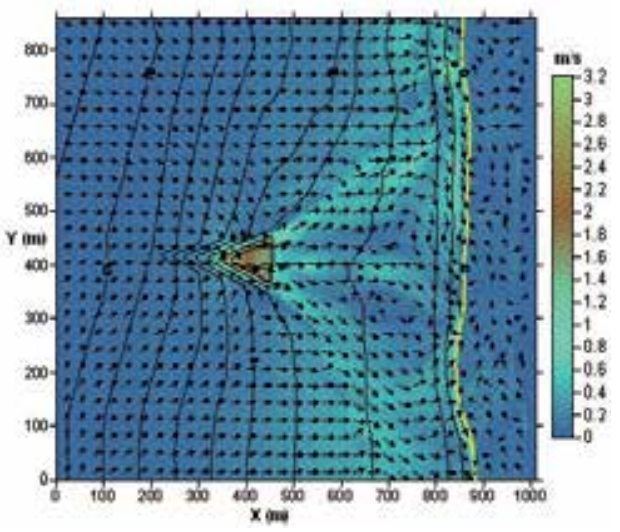

C4: $\mathrm{H}=1.5 \mathrm{~m}, \mathrm{~T}=9 \mathrm{~s}, 6^{\circ}$

Figure 5. Velocity patterns of cases $\mathrm{C} 1$ to $\mathrm{C} 4$ [28]. 
Results of this simulation are described in [29], including the observed phenomena, such as "along the reef, an increase of the wave height is observed, in opposition to the situation without the reef, owing to the decrease of the depth in the reef zone. Moreover, owing to the increase in wave heights, the wave breaking occurs earlier (and in general over the reef) in comparison with the situation without a reef. From Figure 5, it is clear that the presence of the reef significantly alters the wave heights, with the wave height increasing along the reef as a consequence of decreasing water depth".

Figure 5 also shows for all cases that convergent cells appear, indicating a possible sedimentation near the shoreline, suggesting that the chosen geometries (Figure 4) are advantageous for both coastal protection and improving surf conditions. Anyway, as referred in [28], a morphological study should be done in order to confirm these results.

\subsection{HD Serre's standard model}

\subsubsection{Numerical formulation}

The equation system (19) is solved in [7] using an explicit finite-difference method based on the MacCormack time-splitting scheme. For this purpose, the equations are written in the following form:

$$
\begin{gathered}
h_{t}+P_{x}+Q_{y}=0 \\
P_{t}+(u P)_{x}+(v P)_{y}+\left\{[(g+\beta) / 2+\alpha / 3] h^{2}\right\}_{x} \\
=-(g+\beta+\alpha / 2) h \xi_{x}-\tau_{b x}+R \operatorname{div}(h \operatorname{grad} u) \\
Q_{t}+(u Q)_{x}+(v Q)_{y}+\left\{[(g+\beta) / 2+\alpha / 3] h^{2}\right\}_{y} \\
=-(g+\beta+\alpha / 2) h \xi_{y}-\tau_{b y}+R \operatorname{Riv}(h \operatorname{grad} v)
\end{gathered}
$$

where $P=h u, Q=h v, \alpha=d^{2} h / d t^{2} ; \beta=\mathrm{d}^{2} \xi / \mathrm{dt}^{2}$ and the bottom friction terms, $\tau_{b x}$ and $\tau_{b y^{\prime}}$ are obtained through (128):

$$
\tau_{b x}=g \frac{P \sqrt{P^{2}+Q^{2}}}{K^{2} h^{7 / 3}} \text { and } \tau_{b y}=g \frac{Q \sqrt{P^{2}+Q^{2}}}{K^{2} h^{7 / 3}}
$$

In order to apply the MacCormack's method, equations (125) -(127) are split into two systems of three equations throughout the $O x$ and $O y$ directions. The corresponding operators $L x$ and Ly take the following form [7]: 
Operator $L x$

$$
\begin{aligned}
h_{t}+P_{x} & =0 \\
P_{t}+(u P)_{x}+\left\{[(g+\beta) / 2+\alpha / 3] h^{2}\right\}_{x} & =-(g+\beta+\alpha / 2) h \xi_{x}-\tau_{b x}+R P_{x x} \\
Q_{t}+(u Q)_{x} & =R Q_{x x}
\end{aligned}
$$

Operator $L y$

$$
\begin{gathered}
h_{t}+Q_{y}=0 \\
P_{t}+(v P)_{y}=R P_{y y} \\
Q_{t}+(v Q)_{y}+\left\{[(g+\beta) / 2+\alpha / 3] h^{2}\right\}_{y}=-(g+\beta+\alpha / 2) h \xi_{y}-\tau_{b y}+R Q_{y y}
\end{gathered}
$$

Considering the generic variable $F$, the solution at time $(n+1) \Delta t$, for the computational point $(i, j)$, is obtained from the known solution $F_{i, j}^{n}$ through the following symmetric application:

$$
F_{i, j}^{n+1}=\operatorname{Lx}\left(\frac{\Delta t}{4}\right) \operatorname{Ly}\left(\frac{\Delta t}{4}\right) \operatorname{Lx}\left(\frac{\Delta t}{4}\right) \operatorname{Ly}\left(\frac{\Delta t}{4}\right) \operatorname{Ly}\left(\frac{\Delta t}{4}\right) \operatorname{Lx}\left(\frac{\Delta t}{4}\right) \operatorname{Ly}\left(\frac{\Delta t}{4}\right) \operatorname{Lx}\left(\frac{\Delta t}{4}\right) F_{i, j}^{n}
$$

where each operator, $L x$ and $L y$, is composed of a predictor-corrector sequence and $n$ represents a generic time $t$. In the application (135) of eight predictor-corrector sequences, alternately backward and forward space differences are used. After each predictor and each corrector of the application $F$, the values of the velocities $(u, v)$ are updated and the values of the vertical accelerations, $\alpha$ and $\beta$, are recalculated (for details see [7]).

\subsubsection{Real case study}

This model was applied to compute the agitation established in the port of Figueira da Foz, Portugal, for different sea states. This port is 2,250 $\mathrm{m}$ long and $400 \mathrm{~m}$ wide, approximately. Its average depth is of the order of $7 \mathrm{~m}$, with approximately $12 \mathrm{~m}$ throughout the outer port basin.

Two simulations were performed, considering the state of rest as initial condition in both cases, although for different tidal heights. The first case corresponds to the entrance of a sinusoidal wave in the open sea (input boundary), with a mean wave height $H=3.90 \mathrm{~m}$, period $T=15 \mathrm{~s}$, wavelength $\lambda=147 \mathrm{~m}$, and direction $\Phi=260^{\circ} \mathrm{W}$. The second case corresponds to a mean wave height $H=4.80 \mathrm{~m}$, period $T=17.5 \mathrm{~s}$, wavelength $\lambda=173 \mathrm{~m}$, and direction $\Phi=258.2^{\circ} \mathrm{W}$. Figures 6 and 7 show perspective views of the free surface computed in the basin $269.1 \mathrm{~s}$ and $360 \mathrm{~s}$ after excitation. As can be seen, a zone with stronger agitation is observed in the outer harbor in the second case. 


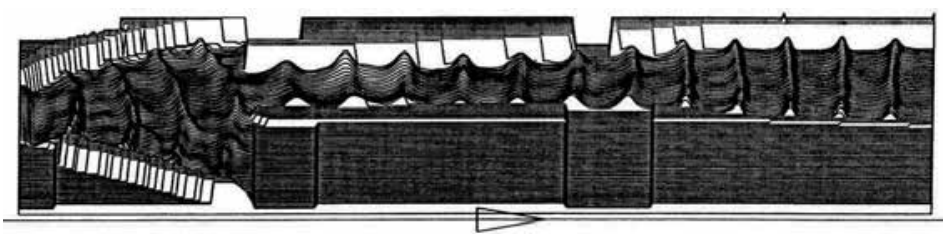

Figure 6. Port of Figueira da Foz. Perspective view of the free surface computed $269.1 \mathrm{~s}$ after excitation, for a mean wave height, $H=3.90 \mathrm{~m}$, period $T=15 \mathrm{~s}$, wavelength $\lambda=147 \mathrm{~m}$ and direction $\Phi=283^{\circ} \mathrm{W}$. Tide height, $3.35 \mathrm{~m}(\mathrm{HZ})$.

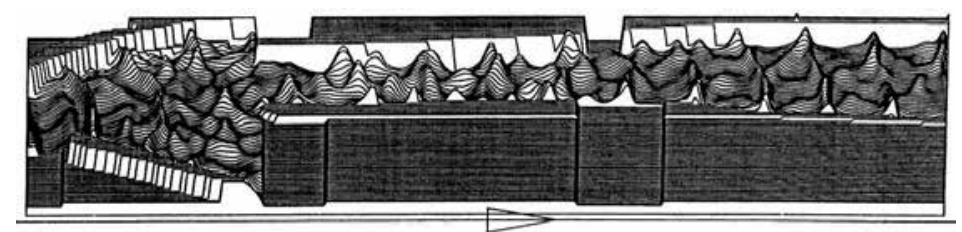

Figure 7. Port of Figueira da Foz. Perspective view of the free surface computed $360 \mathrm{~s}$ after excitation, for a mean wave height $H=4.80 \mathrm{~m}$, period $T=17.5 \mathrm{~s}$, wavelength $\lambda=173 \mathrm{~m}$ and direction $\Phi=258.2^{\circ} \mathrm{W}$. Tide height, $2.65 \mathrm{~m}(\mathrm{HZ})$.

\subsection{HD Serre's extension model}

\subsubsection{Numerical solution}

The equation system (75) is solved using an efficient finite-difference method, whose consistency and stability were tested in [1] and [2] by comparison with a closed-form solitary wave solution of the Serre equations. For this purpose, the terms containing derivatives in time of $u$ are grouped. The final system of three equations is rewritten according to the following equivalent form (SERIMP model) [1, 23$]$ :

$$
\begin{aligned}
h_{t}+ & (u h)_{x}=0 \\
q_{t}+ & \left\{u q-\frac{1}{2}\left[u^{2}+(1+2 \alpha) h^{2}\left(u_{x}\right)^{2}+(1+2 \alpha)\left(\xi_{x}\right)^{2} u^{2}-h \xi_{x}\left(u^{2}\right)_{x}\right]\right\}_{x} \\
& +\left[g(1+\alpha \Omega)+\alpha h u u_{x x}\right] \eta_{x}-\alpha g h h_{x} \eta_{x x}-\beta g \frac{h^{2}}{3} \eta_{x x x} \\
& -\frac{\alpha}{2}\left[\left(h \xi_{x x} u^{2}\right)_{x}+h_{x} \xi_{x x} u^{2}-h \xi_{x x} u u_{x}\right]+\left(\alpha-\frac{\beta}{3}\right) h^{2} u_{x} u_{x x} \\
& +\beta \frac{h^{2}}{3} u u_{x x x}+\tau_{b} /(\rho h)=0 \\
{[1+} & (1+\alpha) \Omega] u-(1+\alpha) h h_{x} u_{x}-(1+\beta) \frac{h^{2}}{3} u_{x x}=q \\
\Omega= & \xi_{x} \eta_{x}+\frac{1}{2} h \xi_{x x}+\left(\xi_{x}\right)^{2}
\end{aligned}
$$


To compute the solution of equation system (75) (values of the variables $h$ and $u$ at time $t+\Delta t$ ), we use a numerical procedure based on the following scheme, itself based on the last equation system (136), for variables $h, q$ and $u$. Knowing all values of $h_{i}$ and $u_{i}, i=1, N$, in the whole domain at time $n \Delta t$, the equations (136c) and (136d) are used to obtain the first values of $q_{i}$ and $\Omega_{i}$ in the whole domain. Then, we continue with the following steps, in which the index $p$ means predicted values (see also [1, 2] and [3]):

1. The first equation (136a) is used to predict the variable values $h_{p i}$ at time $t+\Delta t\left(h_{p i}^{t+\Delta t}\right)$, in the whole domain.

2. The second equation (136b) makes it possible to predict the variable values $q_{p i}$ at time $t+\Delta t$ $\left(q_{p i}^{t+\Delta t}\right)$, taking into account the values $\widetilde{h}_{i}^{t+\Delta t}=0.5\left(h_{i}^{t}+h_{p i}^{t+\Delta t}\right)$, namely for $\Omega_{i}$ in the whole domain.

3. The third equation (136c) makes it possible to compute the mean-averaged velocities $u_{i}^{t+\Delta t}$ at time $t+\Delta t$, taking into account the predicted values $h_{p i}^{t+\Delta t}$ and $q_{p i}^{t+\Delta t}$, namely for $\Omega_{i}$ in the whole domain.

4. The first operation (step 1) is repeated in order to improve the accuracy of the variable values $h_{i}$ at time $t+\Delta t\left(h_{i}^{t+\Delta t}\right)$, using the values $\bar{u}_{i}^{t+\Delta t}=0.5\left(u_{i}^{t}+u_{i}^{t+\Delta t}\right)$ in the whole domain.

5. Finally, the second operation (step 2) is repeated in order to improve the accuracy of the variable values $q_{i}$ at time $t+\Delta t\left(q_{i}^{t+\Delta t}\right)$, taking into account the values $\bar{h}_{i}^{t+\Delta t}=0.5\left(h_{i}^{t}+h_{i}^{t+\Delta t}\right)$ and $\bar{u}_{i}^{t+\Delta t}=0.5\left(u_{i}^{t}+u_{i}^{t+\Delta t}\right)$ in the whole domain.

At each interior point $i$, the first, second and third-order spatial derivatives are approximated through centered differences and the time derivatives are approximated using forward differences. The convective terms $(u h)_{x}$ and $(u q)_{x}$ in equations (136a) and 136b) are approximated through centered schemes in space and time for variables $h$ and $q$. At each time $t$, these terms are written in the following form:

$$
\begin{aligned}
& (u h)_{x}=u_{i}^{t}\left(\frac{h_{i+1}^{t}-h_{i-1}^{t}+h_{i+1}^{t+\Delta t}-h_{i-1}^{t+\Delta t}}{4 \Delta x}\right)+\frac{1}{2}\left(h_{i}^{t}+h_{i}^{t+\Delta t}\right)\left(\frac{u_{i+1}^{t}-u_{i-1}^{t}}{2 \Delta x}\right) \\
& (u q)_{x}=u_{i}^{t}\left(\frac{q_{i+1}^{t}-q_{i-1}^{t}+q_{i+1}^{t+\Delta t}-q_{i-1}^{t+\Delta t}}{4 \Delta x}\right)+\frac{1}{2}\left(q_{i}^{t}+q_{i}^{t+\Delta t}\right)\left(\frac{u_{i+1}^{t}-u_{i-1}^{t}}{2 \Delta x}\right)
\end{aligned}
$$

All finite-difference equations are implicit. Therefore, the solution of system (136) requires, in each time step, the computation of five three-diagonal systems of $\mathrm{N}-2$ equations (steps 1 to 5), which are easily computed using the three-diagonal matrix algorithm (TDMA), also known as the Thomas algorithm. The stability condition to be observed can be expressed in terms of the Courant/CFL number, and is given by: 


$$
C_{R}=\sqrt{g h} \frac{\Delta t}{\Delta x}<1.0
$$

\subsubsection{Boundary conditions}

We often prescribe an influx on the left boundary, usually a mono- or bi-chromatic wave flow. The initial condition for this (mono-chromatic) influx is:

$$
\begin{gathered}
\eta(0, t)=\frac{a}{2}\left\{\sin (\omega t) \tanh \left(t-\frac{\pi}{\omega}\right)+\sin (\omega t)-\frac{\cos (\omega t)}{\omega}\left[1-\tanh ^{2}\left(t-\frac{\pi}{\omega}\right)\right]\right\} \\
u(0, t)=\frac{a}{2} \frac{g}{\omega} \cos (\omega t)\left[\tanh \left(t-\frac{\pi}{\omega}\right)+1\right]
\end{gathered}
$$

where $a$ is the wave amplitude and the tanh is used as a ramp function. It increases smoothly from 0 to 1 , to create a smooth start. For $t$ large, the boundary condition reduces to:

$$
\begin{aligned}
& \eta(0, t)=\frac{a}{2} \sin (\omega t) \\
& u(0, t)=\frac{g}{\omega} \cos (\omega t)
\end{aligned}
$$

If we want to avoid reflections at the right boundary (output), the domain is extended with a damping region of length $L_{\text {dump }}$. In this case, terms like $-m(x) \eta$ and $-m(x) u$ may be added to the continuity (136a) and momentum (136b) equations, respectively. The length of the damped region is chosen such that we do not see any significant reflections.

\subsubsection{Solitary wave travelling up a slope and reflection on a vertical wall}

Experimental data and numerical results are available for a solitary wave propagating on the bathymetry shown in Figure $8[1,2]$. It shows a constant depth before $x=55 \mathrm{~m}$ and a slope 1:50 between $x=55 \mathrm{~m}$ and $x=75 \mathrm{~m}$. An impermeable vertical wall is placed at $x=75 \mathrm{~m}$, corresponding to fully reflecting boundary conditions. A solitary wave of amplitude $0.12 \mathrm{~m}$ is initially centered at $x=25 \mathrm{~m}$. The computational domain was uniformly discretized with a spatial step $\Delta x=0.05 \mathrm{~m}$. A zero friction factor has been considered. Computations were carried out with a time step $\Delta t=0.010 \mathrm{~s}$. Figure 9 compares numerical time series of surface elevation and test data at $x=72.75 \mathrm{~m}$.

Figure 9 shows two peaks; the first one corresponding to the incident wave, and the second to the reflected wave. The extended Serre model predictions for both peaks agree well with the measurements. RMSE values equal to $0.0090 \mathrm{~m}$ and $0.0117 \mathrm{~m}$ were found in first and second peaks, respectively, for the wave height. Regarding the phase, there is a loss of approximately $0.05 \mathrm{~s}$ and of $0.10 \mathrm{~s}$ in those peaks (for details see [1] and [2]). 


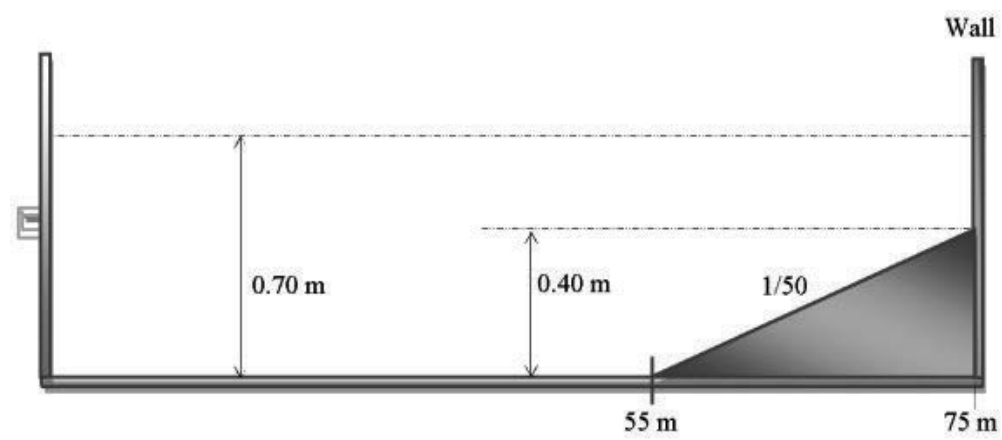

Figure 8. Bathymetry for a solitary wave travelling up a slope and its reflection on a vertical wall (not in scale).

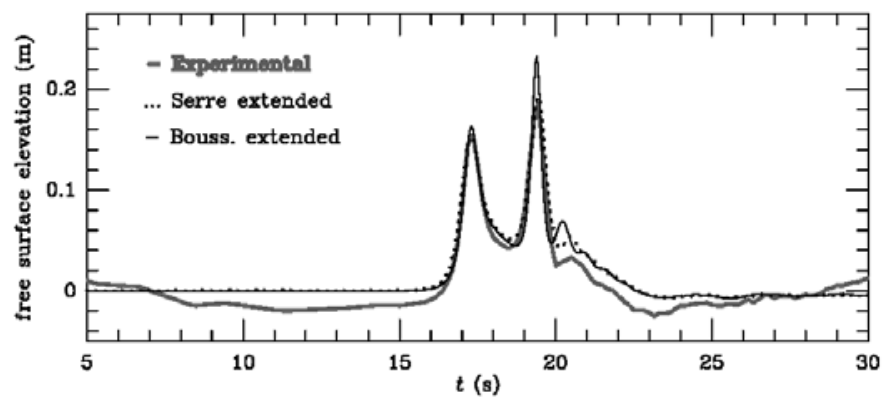

Figure 9. Solitary wave travelling up a slope and its reflection on a vertical wall. Free surface elevation in a depth gauge located at $x=72.75 \mathrm{~m}$. Experimental (- ); Serre extended ( $\cdots)$; Boussinesq extended $(-)[1,2,3]$.

Predictions of the extended Boussinesq equations for both peaks are less accurate. Particularly for the reflected peak, this is overestimated in about $20 \%$. This result is not surprising, given the lower validity of the Boussinesq model for waves of higher relative amplitude. Indeed, this model assumes $O(\varepsilon)<<1$, contrary to the Serre model, which is $O(\varepsilon)=1$. A visual comparison of numerical results of the extended Boussinesq approximation with a similar study performed by [37], using the extended Boussinesq model developed by [30], shows no relevant differences in the graphs (see [1, 2] and [3]).

\subsubsection{Periodic wave over an underwater bar}

Beji and Battjes (1993) [10] conducted experiments in a flume of $0.80 \mathrm{~m}$ wide with a submerged trapezoidal bar. The up- and down-wave bottom slopes of the submerged bar are 1:20 and $1: 10$, respectively. Before and after the bar, the water depth is $0.40 \mathrm{~m}$, with a reduction to 0.10 $\mathrm{m}$ above the bar, as shown in Figure 10. Experimental data obtained in this installation are available in the literature, and can be used for comparisons.

The measured data are compared with numerical results of the extended Boussinesq model (57), with $\alpha=0.1308$ and $\gamma=-0.0076$, and the extended Serre equations (75) (SERIMP model, in [2] and [3]), both improved with linear dispersive characteristics. Comparisons are made in 


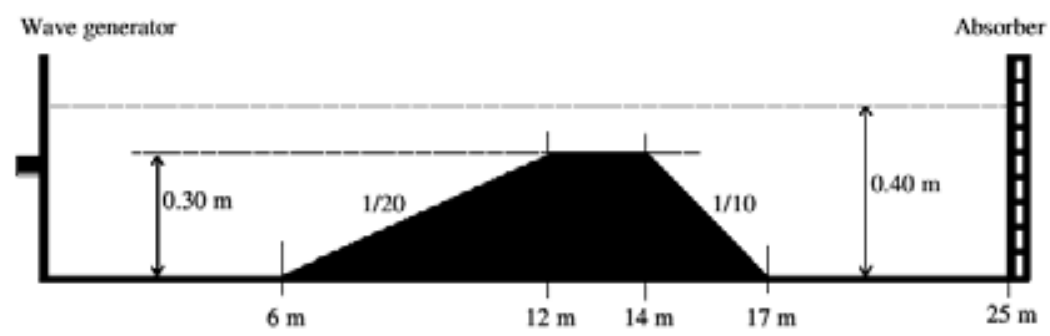

Figure 10. Bathymetry for a periodic wave propagating over a bar (not in scale) [2].

three wave gauges located at $x=10.5 \mathrm{~m}, x=13.5 \mathrm{~m}$ and $x=17.3 \mathrm{~m}$. For this purpose, a regular incident wave case with height $0.02 \mathrm{~m}$, period $T=2.02 \mathrm{~s}$ and wavelength $3.73 \mathrm{~m}$ has been simulated. The computational domain was discretized with a uniform grid interval $\Delta x=0.025$ $\mathrm{m}$. A time step $\Delta t=0.0010 \mathrm{~s}$ was used. Globally, numerical results of the improved Serre and Boussinesq models agree quite well with the measured data (for details see [2] and [3]).

Following is presented a comparison of the standard Serre's model (20) with the extended Serre equations (75) (SERIMP model). The standard Serre's model (20) is only valid for shallow waters, thus under conditions up to $h_{0} / \lambda=0.05$. In this experiment, the dispersion parameter $\left(\sigma=h_{0} / \lambda\right)$ is greater than 0.05 (about 0.11 ) in front and behind the bar, and therefore affects the validity of the numerical outcomes. Due to the fact that over the bar there are very shallow water conditions $(\sigma \approx 0.03)$ the standard Serre equations are used considering the input boundary located at section $x=13.5 \mathrm{~m}$, where the input signal is known (measured data). In this way, results of the Serre's standard model are not influenced, as would happen, by changes arising from the wave propagation before the bar, under intermediate water depths.

Figure 11 shows a comparison of numerical results of the 1HD standard Serre's model (20) with the extended Serre equations (75), considering, in the first case, the input boundary at $x$ $=13.5 \mathrm{~m}$ (gauge signal) (see [2]). The influence of additional terms of dispersive origin included in the extended Serre equations is clearly shown in Figure 11. The standard Serre model results (dashed line) are clearly of lesser quality. It should be noted that this application also demonstrates the good behavior of our numerical model to propagate a complex signal imposed at boundary.

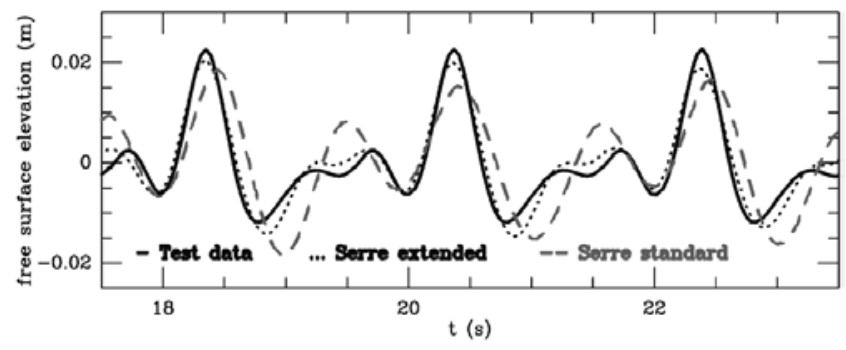

Figure 11. Periodic wave propagating over a bar. Comparison of test data ( - ) with numerical results of the extended Serre model (75) ( $\cdots)$ and the standard Serre equations (20) (---) [2]. 


\section{Conclusions and recommendations}

This work presents some of the most recent methodologies to improve the linear dispersion characteristics of the classical Boussinesq equations for variable depth. A simple procedure to improve the linear dispersive performance of Serre's equations is presented as well.

Extensions of the classical Boussinesq and Serre equations for intermediate depths and deepwater applications are really important, and have been the subject of major developments in recent decades, since these are the conditions typically found in nearshore areas. Application examples to solve real-world problems clearly demonstrate the capabilities and potential of the Boussinesq- and Serre-type models with improved linear dispersion characteristics.

The influence of the dispersion characteristics is clearly evidenced by the generation and propagation of waves in intermediate water depths, as shown here in cases of very demanding applications over bottoms with considerable slopes. The overall agreement of the extended Serre model with improved dispersion characteristics is very good both in shallow water conditions as in intermediate water depths. The influence of additional terms of dispersive origin included in the extended Serre's equations is clearly shown.

This work also shows that current models of Boussinesq type with improved dispersive characteristics are accurate enough for applications in extensive real-world conditions.

Although the contribution of this work to improve the dispersive characteristics of the 1HD standard Serre equations (20) is clear, the author is aware of the need to further improve the dispersion properties of the equation system (75).

An extended version of the two-dimensional system of equations (19) with improved linear dispersion characteristics is being developed and will be published soon.

\section{Author details}

José Simão Antunes do Carmo*

Address all correspondence to: jsacarmo@dec.uc.pt

University of Coimbra, Department of Civil Engineering, Coimbra, Portugal

\section{References}

[1] Antunes do Carmo JS. Boussinesq and Serre type models with improved linear dispersion characteristics: applications. J Hydraulic Res 2013a;51(6):719-27:814090. DOI: $10.1080 / 00221686.814090$. 
[2] Antunes do Carmo JS. Extended Serre equations for applications in intermediate water depths. Open Ocean Eng J 2013b;6:16-25.

[3] Antunes do Carmo JS. Applications of Serre and Boussinesq type models with improved linear dispersion characteristics. In: Jesús MB, Irene A, Alberto P, José MG, Nuno S, Miguel S. (Eds.) Congreso de Métodos Numéricos en Ingeniería; 25-28 June; Bilbao, Spain. Barcelona, Spain: International Center for Numerical Methods in Engineering (CIMNE); 2013c. DOI: ISBN 978-84-941531-4-3.

[4] Antunes do Carmo JS. Closure to "Boussinesq- and Serre-type models with improved linear dispersion characteristics: applications". J Hydraulic Res 2015;53:284-5.

[5] Antunes do Carmo JS, Seabra-Santos FJ. On breaking waves and wave-current interaction on shallow water: a 2DH finite element model. Int J Num Method Fluids 1996;22:429-44.

[6] Antunes do Carmo JS, Seabra-Santos FJ. Wave-current interactions over bottom with appreciable variations in both space and time. Adv Eng Software 2010;41:295-305.

[7] Antunes do Carmo JS, Seabra-Santos FJ, Almeida AB. Numerical solution of the generalized Serre equations with the MacCormack finite-difference scheme. Int J Num Method Fluids 1993b;16:725-38. DOI: 10.1002/fld.1650160805.

[8] Antunes do Carmo JS, Seabra-Santos FJ, Barthélemy E. Surface waves propagation in shallow-water: a finite element model. Int J Num Method Fluids 1993a;16:447-59. DOI: 10.1002/fld.1650160602.

[9] Antunes do Carmo JS, Voorde MT, Neves MG. Enhancing submerged coastal constructions by incorporating multifunctional purposes. J Coastal Conserv 2011;15(4): 531-46.

[10] Beji S, Battjes JA. Experimental investigations of wave propagation over a bar. Coastal Eng 1993;19(1,2):151-62.

[11] Beji S, Nadaoka K. A formal derivation and numerical modelling of the improved Boussinesq equations for varying depth. Ocean Eng 1996;23(8):691-704.

[12] Berkhoff JCW, Booij N, Radder AC. Verification of numerical wave propagation models for simple harmonic linear water waves. Coastal Eng 1982;6:255-79.

[13] Booij N. A note on the accuracy of the mild-slope equation. Coastal Eng 1983;7:191203.

[14] Boussinesq J. Théorie des ondes et des remous qui se propagent le long d'un canal rectangulaire horizontal. J Math Pures Appliq 1872;2(17):55-108.

[15] Dalrymple RA. Model for refraction of water waves. J Waterway Port CoastalOcean Eng 1988;114(4):423-35. 
[16] Gobbi MFG, Kirby JT, Wei G. A fully nonlinear Boussinesq model for surface waves. Part 2. Extension to O(kh)4. J Fluid Mechanics 2000;405:181-210.

[17] Green AE, Naghdi PM. A derivation of equations for wave propagation in water of variable depth. J Fluid Mechanics 1976;78(2):237-46.

[18] Hsiao SC. Permeable effects on nonlinear water waves [thesis]. Cornell University: 2000.

[19] Hsiao SC, Liu PLF. Permeable effects on nonlinear water waves. In: Royal Society of London, editor. London. London:2002.

[20] Kirby JT. A note on linear surface wave-current interaction over slowly varying topography. J Geophys Res 1984;89(C):745-47.

[21] Kirby JT, Dalrymple RA. A parabolic equation for the combined refraction-diffraction of stokes waves by mildly varying topography. J Fluid Mechanics 1983;136:43566.

[22] Liu PLF. Model equations for wave propagation from deep to shallow water. In: Liu PLF, (Ed.) Advances in Coastal Engineering. World Scientific. 1994, pp. 125-157.

[23] Liu ZB, Sun ZC. Two sets of higher-order Boussinesq-type equations for water waves. Ocean Eng 2005;32:1296-310.

[24] Lynett P. Nearshore wave modeling with high-order Boussinesq-type equations. J Waterway Port Coastal Ocean Eng 2006;132(5):348-57.

[25] Lynett P, Liu PLF. (Eds.) Modeling Wave Generation, Evolution, and Interaction with Depth Integrated, Dispersive Wave Equations COULWAVE Code Manual. 1st ed. Ithaca, NY: Cornell University Long and Intermediate Wave Modeling Package; 2002.

[26] Lynett P, Liu PLF. Linear analysis of the multi-layer model. Coastal Eng 2004;51:43954. DOI: 10.1016/coastaleng.2004.05.004.

[27] Madsen PA, Murray R, Sørensen OR. A new form of the Boussinesq equations with improved linear dispersion characteristics. Coastal Eng 1991;15(4):371-88.

[28] Mendonça AM, Fortes CJ, Capitão R, Neves MG, Moura T, Antunes do Carmo JS. Wave hydrodynamics around a multi-functional artificial reef at Leirosa. J Coastal Conserv 2012a;16(4):543-53. DOI: 10.1007/s11852-012-0196-1.

[29] Mendonça AM, Fortes CJ, Capitão R, Neves MG, Antunes do Carmo JS, Moura T. Hydrodynamics around an Artificial Surfing Reef at Leirosa, Portugal. J Waterway Port Coastal Ocean Eng 2012b;138(3):226-35. DOI: 10.1061/(ASCE)WW. 1943-5460.0000128.

[30] Nwogu O. Alternative form of Boussinesq equations for nearshore wave propagation. J Waterway Port Coastal Ocean Eng 1993;119:618-38. 
[31] Press WH, Flannery BP, Teukolsky SA. Numerical Recipes. Cambridge University Press. 1989, pp. 569-72.

[32] Saint-Venant B. Theory of unsteady water flow, with application to river floods and to propagation of tides in river channels. Computes Rendus Acad. Sci., Paris. 1871;73:148-54, 237-40.

[33] Seabra Santos FJ. Contribution a l'ètude des ondes de gravité bidimensionnelles en eau peu profonde [thesis]. Université Scientifique et Médicale et Institut National Polutechnique de Grenoble, France:1985.

[34] Seabra Santos FJ. As aproximações de Wu e de Green \& Naghdi no quadro geral da teoria da água pouco profunda (in Portuguese). In: APRH, editor. Simpósio LusoBrasileiro de Hidráulica e Recursos Hídricos (4ํ SILUSBA); 14-16 June; Lisbon, Portugal, LNEC, Lisbon:1989. pp. 209-219.

[35] Serre F. Contribution à l'étude des écoulements permanents et variables dans les canaux. La Houille Blanche 1953;8:374-88.

[36] Walkley MA. A Numerical Method for Extended Boussinesq Shallow-Water Wave Equations [thesis]. Leeds, UK: University of Leeds, School of Computer Studies:1999.

[37] Walkley MA, Berzins M. A finite element method for the one-dimensional extended Boussinesq equations. Int J Num Method Fluids 1999;29:143-57.

[38] Wei G, Kirby JT. Time-dependent numerical code for extended Boussinesq equations. J Waterway Port Coastal Ocean Eng 1995;121(5):251-61.

[39] Wei G, Kirby JT, Grilli ST, Subramanya R. A fully nonlinear Boussinesq model for surface waves. I. Highly nonlinear, unsteady waves. J Fluid Mechanics 1995;294:7192.

[40] Woo S-B. Finite element modeling of the fully-nonlinear extended Boussinesq equations [thesis]. Ithaca, NY: Cornell University:2002. 
Chapter 3

\title{
CFD-Based Investigation of Wind-Strokes over Highway Bridge Section
}

\author{
Medzid Muhasilovic, Kenan Imsirpasic, Karel Ciahotny and Brano Sirok \\ Additional information is available at the end of the chapter
}

http://dx.doi.org/10.5772/61928

\begin{abstract}
There is an almost everlasting debate on the possibilities of the investigation tools for their applications in a prospective fashion (while solving the engineering tasks) and against this fact - on the use of these engineering tools while correcting the existing technology problems. A unique chance to compare these needs of research in traffic intentions (while setting the modern road communications through southern Bosnia and Herzegovina) with the natural occurrences in the atmosphere (such is a strong north wind in this geographic region) offers us the highway section Pocitelj-Zvirovici. Exactly in such cases (and before the actual construction of this highway bridge) "for the sake" of prospective engineering, the CFD mechanism (the "toolkit" for performing the computational fluid dynamics) was applied to engage this atmospheric problem. Both as steadystate explorations (while applying the $k-\varepsilon$ turbulence treatment) and as the timedependent CFD-based mode, we explored the wind-strokes of $10 \mathrm{~m} / \mathrm{s}, 20 \mathrm{~m} / \mathrm{s}, 30 \mathrm{~m} / \mathrm{s}$ and $40 \mathrm{~m} / \mathrm{s}$, expected but certainly unwanted strong gaseous flows over the bridge, detecting in this way the traffic safety edge points. The results coming from the explorations performed by the CFD tool are explained and discussed.
\end{abstract}

Keywords: Traffic safety, Highway bridge, Wind-stroke, CFD (computational fluid dynamics)

\section{Introduction}

While establishing the modern road and railway infrastructure that is not only impressive in the construction way but is also needed for accurate and important trafficking, one confronts the reality that is always surrounding such objects: the nature of our planet [1-4]. In spite of evaluations [5-8] and certain suggestions[9], every new object of traffic infrastructure that is exposed to rather strong atmospheric influences is presenting the safety risks and calls for 
exploring in a large-scale fashion. Such investigations, due to the ever-stronger software and hardware tools [10-12], are performed not only through the physical measuring [13] and scaled testing [14] but also more frequently by applying the CFD (computational fluid dynamics)based approach[12]. The latter research mode [15] did find application in wind exploring [16, 17] and traffic safety [18] which is the research pathway of the work presented in this paper, offering very satisfying results accomplished in its attempts at "prospective engineering" for particular explored cases of fluid phenomena [19].

All of these research attempts that have been brought up into the CFD community do report on good capability of the numerical approaches used in handling the reactive flows in straight, enclosed traffic infrastructure. Besides the slight denivelation of a few percent, the geometry of the arbitrary objects of interest was relatively a simple one.

Therefore, the aim of the study is to explore the (accidental) wind-strokes over such a bridge that, as a segment of to-be-constructed highway, for sure turns up as an element of this modern traffic road communication and hence suggests some countermeasures serving the overall traffic safety.

\section{Numerical approach}

\subsection{Treatment of turbulence: Mathematical model in this study}

For turbulence-modelled conservation equations, for mass and momentum, employing a timeaveraged $k-\varepsilon$ turbulence model (a CFD mode that was applied in this study as well), the governing integro-differential equations must be discretised in space only [20,21]. These equations, together with the equations of state for an ideal gas, form here a closed set of coupled equations. These are again discretised and solved on a three-dimensional, finite-volume Cartesian mesh. In choosing the numerical method, we [22] rely on the standard of the finite volumes [21, 23, 24]. The spatial discretisation of time-independent equations employs a segregated solution method. The linearised equations result in a system of linear equations for each cell in the computational domain, containing the unknown variable at the cell centre as well as the unknown values in surrounding neighbour cells. This mechanism for a scalar transport equation [22] is also used to discretise the momentum equations; in the same mode for the pressure field (if face mass fluxes were known) and the velocity field will be obtained in same way as well.

In cases where the pressure field and face mass fluxes are not known, FLUENT (the software package applied in this research attempt) uses a co-located scheme, whereby pressure and velocity are both stored at cell centres. A need for interfacial values includes an application of an interpolation scheme to compute pressure and velocity out of cell values. The integration over the arbitrary volume (a cell in a computational domain) can be performed and is discretised through an arbitrary surface of a face.

Executing these numerical steps, the equations can express the state for each other cell in the computational grid. This again will result in a set of algebraic equations with a sparse coefficient matrix. In this way, the segregated solver is handling "the updating" of a single variable 
field, by considering all the cells of the domain at the same time, solving the governing equations sequentially (segregated one from another). Subsequently, the next field of another variable will be solved by again considering the entire cells at the same time. The computational loop for the converged solution had about 5,500 iterations.

\section{Procedure of investigation}

The estimation of the boundary conditions in this CFD-based investigation was supported by the experience of some previous studies and so were the bridge surrounding space characterised as open (pressure) atmospheric boundaries with minor pressure increase or pressure drop of $2 \mathrm{~Pa}$, respectively. All zones formed around the road bridge an open (pressure) boundary, which was used for initialising the values for the velocity and pressure in the computational domain, while the global temperature was set to $293 \mathrm{~K}$.

The bridge body and bridge road elements as well were presumed to be nonadiabatic in the area where the objects of interest (the investigated bridge crown) are situated. This decision was based on some reality-oriented investigation on modern bridge construction, denoting the thermal conductivity of a reinforced concrete to be $\lambda=2,3 \mathrm{~W} / \mathrm{mK}$.

\subsection{The explored object of interest}

The cross-section shapes of this highway viaduct are distinguished as those between the major carrier pylons. Further, the bridge crown shapes are mounted onto the "bridge legs" of this traffic steady object. Standing with the angle of ca $3.1^{\circ}$, the road treks of this bridge have the bow-length of $954 \mathrm{~m}$ and their arch radius is $983 \mathrm{~m}$. Going partly over the river bed and partly over the terrain valley, the highway bridge "Pocitelj-Zvirovici" demonstrates its highest section to be $96 \mathrm{~m}$. The wide range between the six major pylons is set to $147 \mathrm{~m}$.

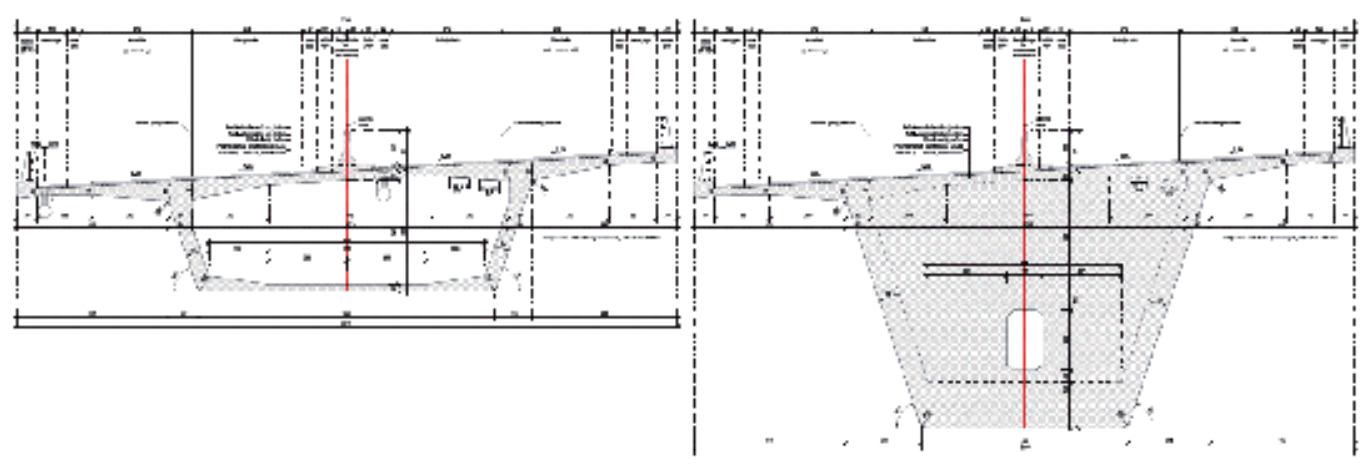

Figure 1. The cross section of the bridge crown in the free air as well as over the pylon 


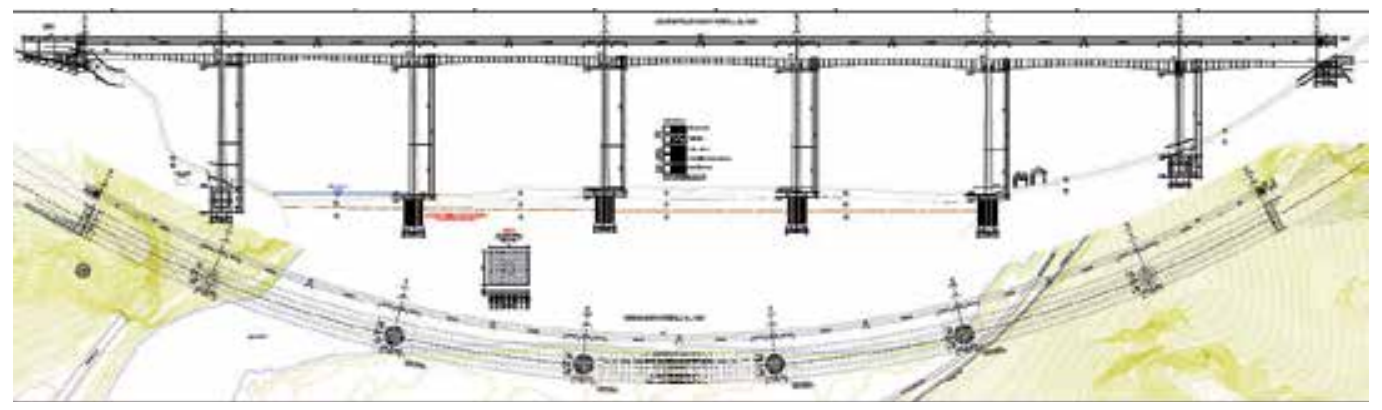

Figure 2. The constructive disposition of the highway bridge "Pocitelj-Zvirovici" in southern BH

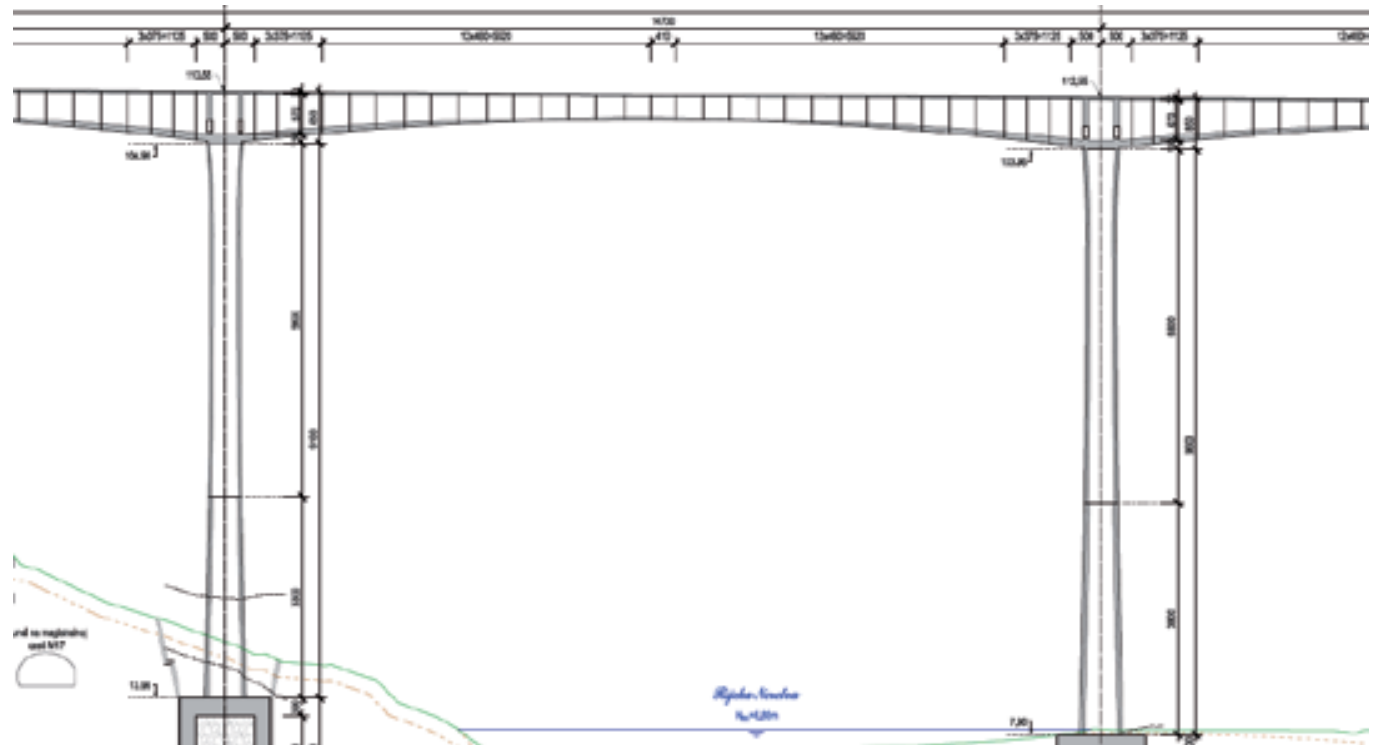

Figure 3. A side view of one of the bridge sections while crossing the river Neretva and following its shore terrain

\subsection{Computational domain}

The area in which the computation with applied mathematical model approach and additional numerical discretisation was performed is the very volume that a fluid can take without the "walls", where the solid body is the shape of the explored road bridge. Therefore, the computational domain of the section "Pocitelj-Zvirovici" was set to be 30m x 22m x 14,5 m.

\subsubsection{The highway bridge "Pocitelj-Zvirovici"}

The mesh of this computational domain (Figs. 4 and 5) is characterised through tetrahedral cells and hexahedral prisms of a random structure. In this case, a denser grid was also applied 
in the area around the zones where particular mechanical fluid phenomena are expected, having so more grid points to support the major occurrences.

Such an unstructured mesh (sized here to $350 \mathrm{~mm}$ ) was installed in such zones of whole computational domain. However, the following parts of the 954-m-long bridge are also meshed with unstructured hexahedral cells in the explained way, having subsequent increased cell size up to $400 \mathrm{~mm}, 800 \mathrm{~mm}$ and 1,200 $\mathrm{mm}$ - as the distance from the bridge bottom towards the open space was growing.

The "Pocitelj-Zvirovici" bridge body and the road elements were in the computational domain defined as nonadiabatic walls. The fluid domain is air, with the ambient conditions and no fluid movement, onto which the wind-strokes were expected. The computational fluid sides were designed as opened pressure boundaries.

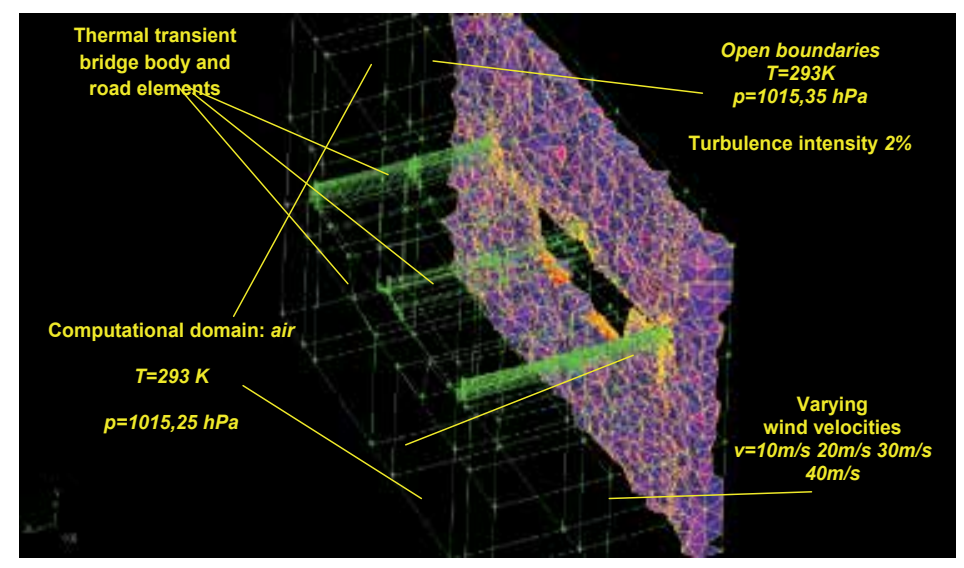

Figure 4. The unstructured hexahedral mesh is applied over the "Pocitelj-Zvirovici" highway bridge - here, at one fourth of its length, around the zone of the fuel pool

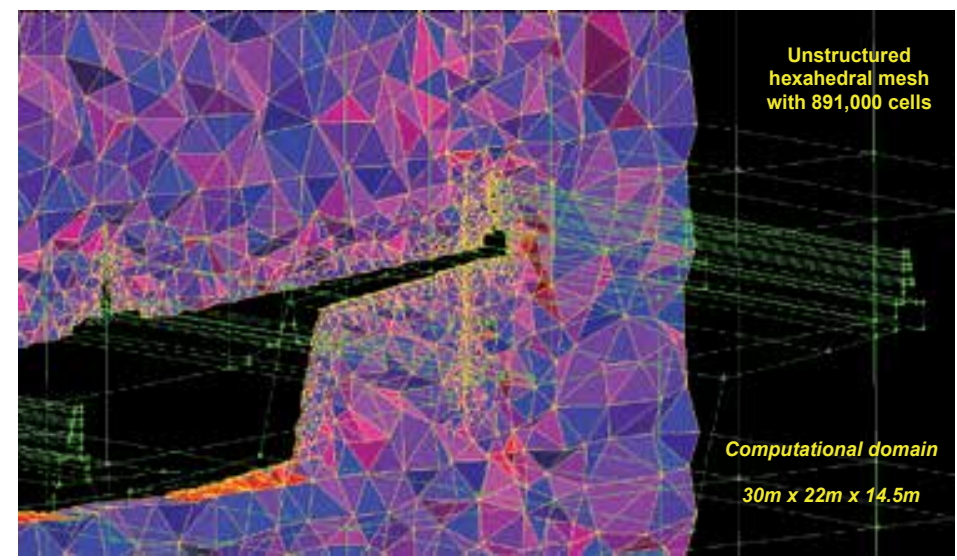

Figure 5. The meshing detail over the "Pocitelj-Zvirovici" highway bridge - here, demonstrating the solution around the road fence 


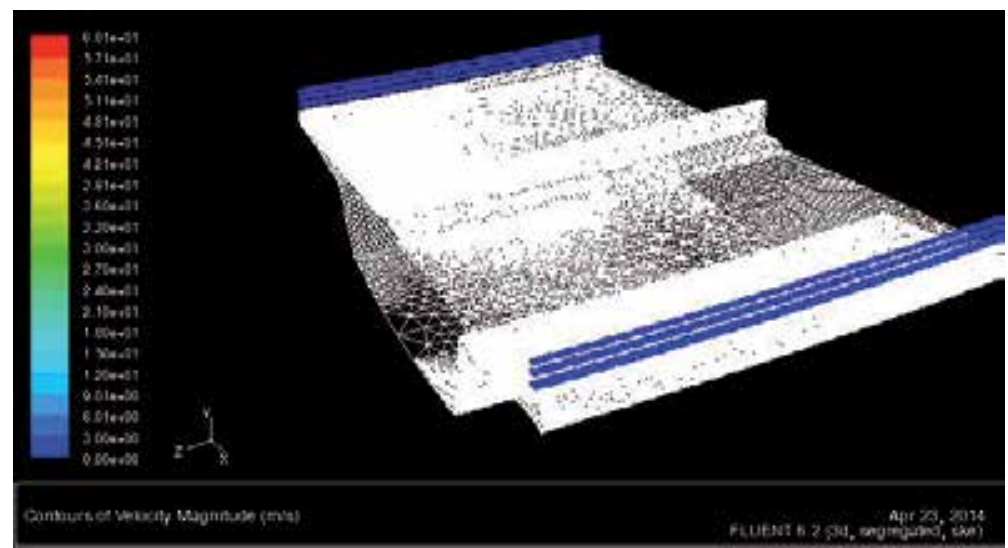

Figure 6. The meshing detail of the Bridge segment "Pocitelj-Zvirovici"

\section{Discussion and outlook}

Consulting the meteorological survey of the State Weather Service of Bosnia and Herzegovina[25], we performed several investigation scenarios by varying computationally aided, simulative conditions of the unwanted wind-strokes: from both south and north side of the highway bridge "Pocitelj-Zvirovici". Here (Fig. 4), we have chosen the sketch that presents CFD calculation of a (not seldom) $40 \mathrm{~m} / \mathrm{s}$ stroke, from the more influencing north wind. Taken alone, this result (that was presented by the time-independent Reynolds-averaged NavierStokes numerical research) points up the need for a wind flow disturbing panel fences along the roadsides [26].

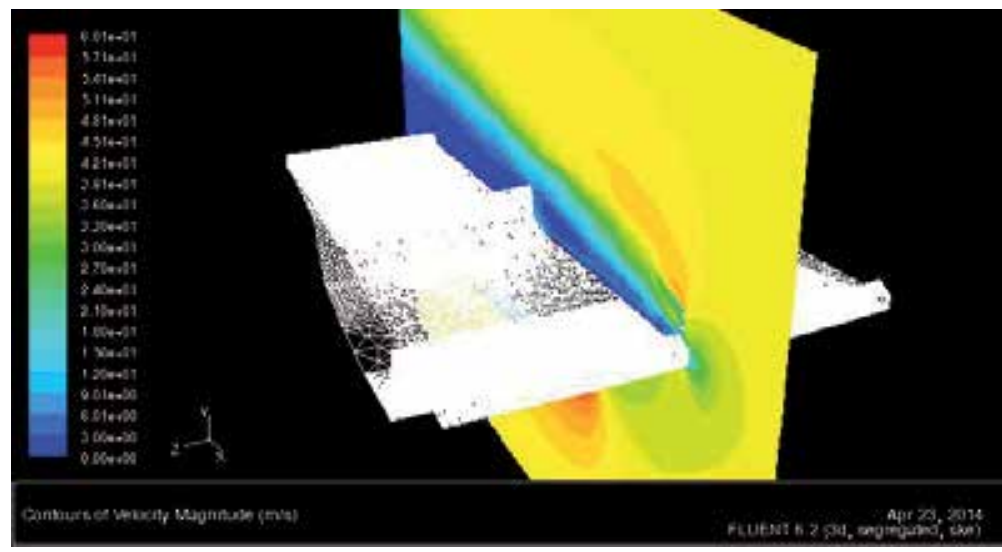

Figure 7. The computationally aided research of the wind-stroke, with the velocity of above $140 \mathrm{~km} / \mathrm{h}$ : after the "Staupunkt" zones, the air, as every Newtonian fluid [6] that eventually runs around the obstacle and increases its propagation velocity 
Even "weaker", sudden wind occurrences (out of $10 \mathrm{~m} / \mathrm{s}$ - Fig. 8) that do turn up more frequently in the roses of wind for the geographic region, where this highway viaduct is to be constructed, do present the traffic hazard for the infrastructure element in this road communication. Our investigation performed in CFD mode does correlate with modern studies [27] and does report [28] on the importance [1] of taking into account security and safety.

During this research, we were able to perform the importance of a prospective engineering approach in treating the (gaseous) fluid phenomena in a large-scale mode. In this case, the traffic safety profits out of our findings - where again those results are "pushing" towards further prospective investigations. Exactly CFD-based research obligates to continuing investigation of the mentioned highway bridge, where the whole set of interacting answers and their questions could be satisfied: by applying another turbulence treatment (while using the LES model, for instance), by changing the regime of the numerical approach in another time fashion, by discretising the computational domain while using the other basic-cell shapes, and by applying different real-case scenarios based upon the atmospheric data from the statistics of the meteorology services.

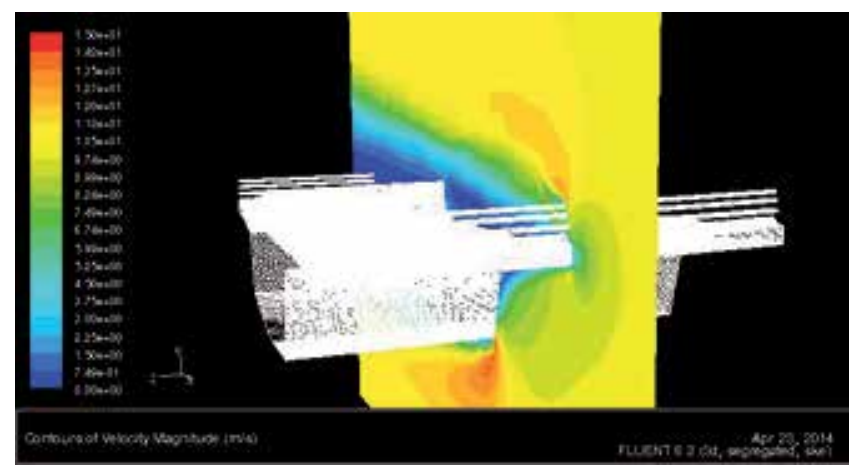

Figure 8. Even the "weaker" wind-stroke of some $36 \mathrm{~km} / \mathrm{h}$, after passing its "Staupunkt" zones, increases its velocity propagation and according to the CFD-based observation, reaches the wind fluctuation of above $50 \mathrm{~km} / \mathrm{h}$

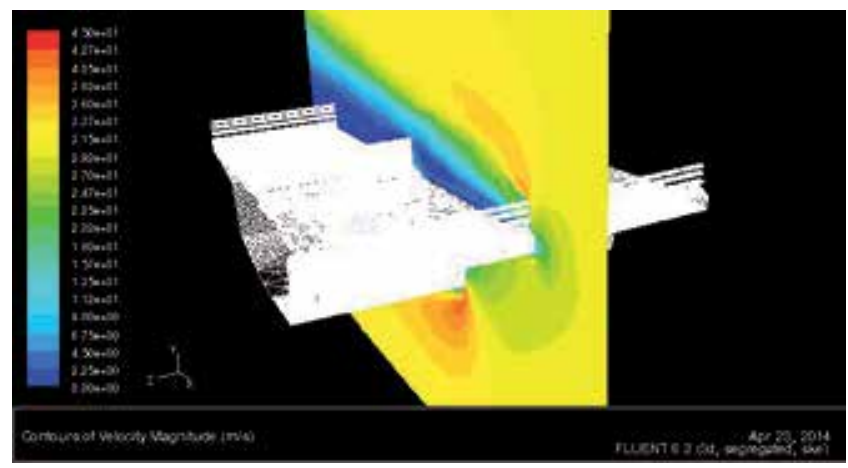

Figure 9. Averaged by its occurrence, the wind-stroke in a range of $70 \mathrm{~km} / \mathrm{h}$ is due to the climatic changes, one of the frequent and unwanted air blows over the viaduct infrastructure throughout Europe 


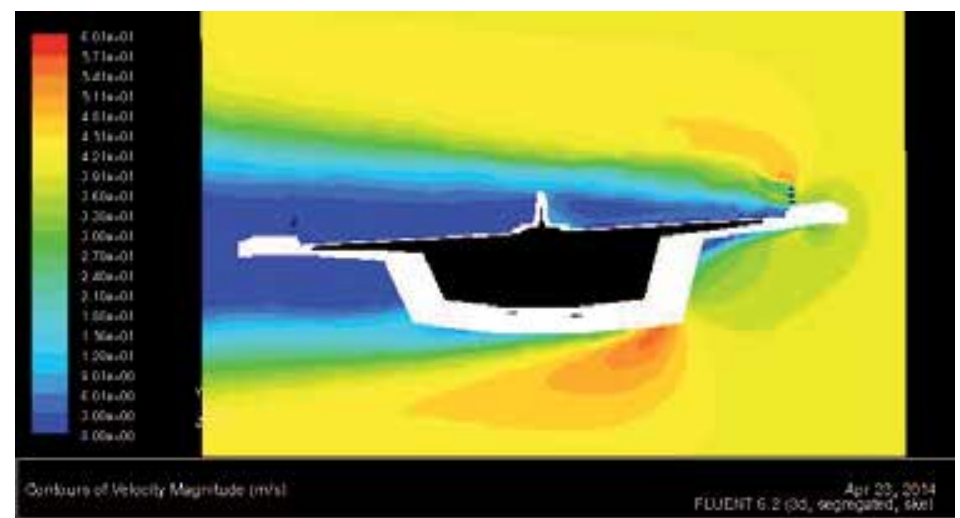

Figure 10. The mentioned dangerous increase of wind velocity after the obstacle in the form of traffic road security fence is visible

The safety issue caused by atmospheric occurrences varies in different parts of Europe (let alone in various parts of the world); what is inviting or even more is it is "provoking" us to further explore on these traffic safety issues. The landscape of the geographic regions where the future (both road and rail) infrastructure is to be constructed and to them belonging particular meteorological circumstances offers a unique perspective for each of these traffic objects. Modern hardware and software [29] developments [30] in the last 20 years [31] endorsed the computational modelling [32] into the modern and very reliable CFD toolkit [29] for scientific engineering and research [33].

\section{Author details}

Medzid Muhasilovic ${ }^{1 *}$, Kenan Imsirpasic ${ }^{1}$, Karel Ciahotny ${ }^{2}$ and Brano Sirok ${ }^{3}$

*Address all correspondence to: muhasilovic@gmail.com

1 IPSA-Institute, Sarajevo, Bosnia and Herzegovina

2 VSCHT, Vysoka Skola Chemicko Technologicka, Praha, Czech Republic

3 Univerza v Ljubljani, Fakulteta za Strojnistvo, Ljubljana, Slovenija

\section{References}

[1] H.K. Lorenzo Procino, G. Bartoli, A. Borsani, Wind barriers on bridges: the effect of wall porosity, in BBAA VI International Colloquium on: Bluff Bodies Aerodynamics \& Applications. 2008. Milano, Italy. 
[2] D. Hembre, C. Otto, J. Payette, G. Pingree, The 50th anniversary of the Golden-Gate Bridge, in Laboratory for Construction Technology, Graduate School of Design 1988, Harward University: Cambridge, Massatchussets, USA.

[3] P. Bowers, J. Boneck, Charleston's bridges cross troubled waters, in Charleston City Paper2012: Charleston, South Carolina, USA.

[4] E. Simiu, P. Vickery, A. Kareem, Relation between Saffir-Simpson hurricane scale wind speeds and peak 3-s gust speeds over open terrain. Journal of Structural Engineering, 2007. 133.

[5] A. Greeman, Calls for wind shields after lorry falls off bridge, in New Civil Engineer, www.nce.co.uk2005.

[6] Hideharu Yagi, Fumitake Yoshida, Gas absorption by newtonian and non-newtonian fluids in sparged agitated vessels. Industrial \& Engineering Chemistry Process Design and Development, 1975. 14(4): p. 15.

[7] C. Airong, Y. Qingzhong., Z. Xigang, M. Rujin, Z. Zhiyong, Aerodynamic problems of a super-long span cable-stayed bridge, in IABSE SYMPOSIUM2005: Lisbon, Portugal.

[8] T. Martin, I.A. MacLeod, The Tay Rail Bridge disaster revisited, in Proceedings of the Institution of Civil Engineers 2004. p. 5.

[9] D.E. Newland, Vibration of the London Millennium Footbridge: Part 1 - Cure, in Department of Engineering 2002, University of Cambridge: CAMBRIDGE CB2 1PZ, UK.

[10] S. Muzaferija, D. Gosman, Finite-volume CFD procedure and adaptive error control strategy for grids of arbitrary topology. Journal of Computational Physics, 1997. 138(2).

[11] S. Svaic, I. Boras, M. Andrassy, A numerical approach to hidden defects in thermal nondestructive testing. Journal of Mechanical Engineering, 2007. 53(3): p. 165.

[12] L. H. Cheng, T.H. Ueng, C. W. Liu, Simulation of ventilation and fire in the underground facilities. Fire Safety Journal, 2001. 36(6): p. 597-619.

[13] J. Modic, Porocilo o meritvah pri pozernem preizkusu v cestnem tunelu SENTVID, 2008, University in Ljubljana, Mechanical ENgineering: Ljubljana, Slovenia.

[14] L. ZhouI, Y. Ge., Wind tunnel test for vortex-induced vibration of vehicle-bridge system section model. Journal of the Brazilian Society of Mechanical Sciences and Engineering, 2008. 30(2).

[15] A. Kareem, Numerical simulation of wind effects: a probabilistic perspective. Journal of Wind Engineering and Industrial Aerodynamics, 2008. 96: p. 25.

[16] T. Kijewski-Correa, e.a., Validating wind-induced response of tall buildings: synopsis of the Chicago full-scale monitoring program. Journal of Structural Engineering, 2006. 132(10).

[17] D.-K. Kwon, T. Kijewski-Correa, A. Kareem, e-Analysis of high-rise buildings subjected to wind loads. Journal of Structural Engineering, 2008. 134: p. 1139. 
[18] X. Chen, A. Kareem, Identification of critical structural modes and flutter derivatives for predicting coupled bridge flutter. Journal of Wind Engineering and Industrial Aerodynamics, 2008. 96: p. 14.

[19] X. Chen, A. Kareem, Revisiting multimode coupled bridge flutter: some new insights. Journal of Structural Engineering, 2006. 132(10).

[20] http://www.fluent.com.

[21] H. K. Versteeg, W. Malalasekera, An Introduction to Computational Fluid Dynamics, 1995, London: Longman Group Ltd.

[22] M. Muhasilovic, M. Deville, Tunnel-curvatire's influence on the propagation of the consequences of large-scale accidental fire - a CFD-investigation. Turkish Journal of Engineering and Environmental Sciences, 2007. 31: p. 391.

[23] Hirsch, C., Numerical Computation of Internal and External Flows. Vol. I. 1988, Chichester Brisbane Toronto New York: John Wiley \& Sons. p. 515.

[24] M. Peric, J.H. Ferziger, Computational Methods for Fluid Mechanics, 2001, Berlin: Springer Verlag. p. 423.

[25] http://www.fhmzbih.gov.ba/.

[26] K. Ilic, Projekt i Izvedba Viadukta "Hreljin" na Autocesti Rijeka-Zagreb, in Inzenjerski Projektni Zavod, Zagreb 2008.

[27] H. Kozmar, L. Procino, G. Bartoli, A. Borsani, Wind barriers on bridges: effects of wind incidence angle on flow field characteristics, in The Seventh Asia-Pacific Conference on Wind Engineering 2009: Taipei, Taiwan.

[28] S. James, Forth Replacement Crossing Study - Windshielding on Bridge Options, 2007, Jacobs UK Ltd.

[29] S. R. Lee, H.S. Ryou, A numerical study on smoke movement in longitudinal ventilation tunnel fires for different aspect ratio. Building and Environment, 2006. 41(6): p. 719-725.

[30] M. Deville, A.D., COSMASE, 1996: Lausanne, Switzerland.

[31] J. S. M. Lee, W.K. Chow., Numerical studies on performance evaluation of tunnel ventilation safety systems. Tunneling and Underground Space Technology, 2003. 18(5): p. 435-452.

[32] S. Muzaferija, V. Seidl, A. Kneer Parallel multidimensional calculation of steady-state and time-dependent flows with combustion, in Euro-Par '97: the Third International Euro-Par Conference on Parallel Processing 1997.

[33] C. C. Hwang, J.C. Edwards, The critical ventilation velocity in tunnel fires - a computer simulation. Fire Safety Journal, 2005. 40(3): p. 213-244. 


\title{
Chapter 4
}

\section{Computational Fluid Dynamics Achievements Applied to Optimal Crop Production in a Greenhouse}

\author{
Jorge Flores-Velázquez, Abraham Rojano, Adriana Rojas-Rishor and \\ Waldo Ojeda Bustamante
}

Additional information is available at the end of the chapter

http://dx.doi.org/10.5772/61006

\begin{abstract}
Computational fluid dynamics has been successfully used in protected agriculture to simulate greenhouse weather as physical processes. The variables involved are velocity, wind direction related to either absolute or relative humidity, temperature as well as deficit vapor pressure, and carbon dioxide, among others. The research evolution is changing from the traditional validation of new designs and management to testing efficient production with less environmental pollution. This work points out this kind of assessment based on the physical principles of conservation of mass, momentum, and energy. Constitutive relationships like Darcy-Forchheimer porosity model in the momentum equation as well as the geometry and physical properties of the materials involved are needed to fulfill the particular solutions of temperature, wind, and humidity. This chapter is enhanced by the effect of solar radiation in more processes like crop transpiration with dynamical meshes and condensation.
\end{abstract}

Keywords: Computational fluid dynamics, greenhouse, climate variables, crop

\section{Introduction}

Intensive production systems are established in order to obtain the highest yields in the evolution of agriculture as the primary sector. One of the techniques of crop production is protected agriculture. Greenhouse has helped to increase production, improve quality, and provide sustained and profitable yields. By confining the environment in a greenhouse, the local atmosphere must fit well for cultivation. Making these adjustments sometimes cannot be easy, because the components of the atmosphere (temperature, humidity, $\mathrm{CO}_{2}$ ) are intrinsically linked. The Computational Fluid Dynamics (CFD) is a tool that provides alternatives for 
analysis and management of greenhouse environment from a numerical point of view. CFD has advantages over other simulators, because it allows spatial and temporal analysis of the dynamics of the air inside the greenhouse, by introducing local climatic variables as starting conditions. The mathematical approach is based on physical laws expressed as partial differential equations dealing with the dependent variables as unknowns. Therefore, solving this system of equations allows either to see specific details or monitoring of greenhouse environment.

\section{Models applied to protected agriculture}

The inside greenhouse climate depends mainly on local environmental factors, geometry, as well as the type of materials, but they all effect the global distributional variables like: inner air temperature, exterior temperature, relative humidity, solar radiation, and wind speed and direction, further the case of the crop in the greenhouse changes transpiration rate, steam and carbon dioxide. The mentioned variables are complex factors which force solutions for a better approximation.

\subsection{Lumped mechanistic models}

Mechanistic models are based on physical equations. A general representation of this model is shown in Eq. (1). The main equation calculates the temperature inside the greenhouse depending on the factors described on the right side:

$$
V_{\text {grh }} \rho_{\text {air }} c p_{\text {air }} \frac{d T_{\text {in }}}{d t}=Q_{\text {sun }}-Q_{\text {vent }}-Q_{\text {conv }}+Q_{\text {sol }}
$$

Depending on the components of the greenhouse, a more complete equation can be formulated as follows:

$$
\begin{aligned}
& V_{\text {grh }} \rho_{\text {air }} c p_{\text {air }} \frac{d T_{\text {in }}}{d t}=\varepsilon^{*} A_{c l} * R a d_{\text {out }}- \\
& -F_{v}^{*} \rho_{\text {air }} * c p_{\text {air }}\left(T_{\text {in }}-T_{\text {out }}\right)-\alpha_{c l}\left(T_{\text {in }}-T_{\text {out }}\right)+\alpha_{\text {soil }}\left(T_{\text {in }}-T_{\text {soil }}\right)
\end{aligned}
$$

In Eq. (1) and (2) are avoided culture or transport of energy by radiation because it does not provide low temperature. Eq. (2) describes the energy representing the greenhouse environment taking into account the ground effect.

\subsection{Black box model (ARX)}

The black box model allows statistical description based on the outputs, given inputs on a limited range. The model only uses data obtained from direct measurements and is 
considered an empirical approach. So this system also provides a description of the climate of a greenhouse [1].

In general, a mathematical way to write the ARX model with one input and one output is represented in Eq. (3)

$$
\begin{aligned}
& y(t)+a_{1} y(t-1)+\ldots . .+a_{n a} y(t-n a)= \\
& =b_{1} u(t-n k)+b_{2} u(t-n k-1)+\ldots \ldots .+b_{n b} u(t-n k-n b+1)+e(t)
\end{aligned}
$$

where

$y(t)=$ output of the ARX model for $t=t, t_{-1} t_{-n a} \cdots$

$u(t)=$ input of the ARX model for $t=t_{-n k}, t_{-n k-1} \cdots t_{-n k-n b+1}$

na $=$ number of time steps out in the past

$\mathrm{nb}=$ number of time steps in the past entry

$\mathrm{nk}=$ entry delay, $\mathrm{u}(\mathrm{t})$, the output $\mathrm{y}(\mathrm{t})$

\subsection{Artificial intelligence techniques: artificial neural networks, fuzzy logic-based systems, evolutionary algorithms}

Artificial neural networks are nonlinear empirical models, which have been successfully used for modeling greenhouse climate [2]. The nonlinear models are suitable candidates for dealing with complexities observed in the greenhouse behavior as moisture. However, in reference [3] mentions that require a large amount of data and time. Perhaps the best use of neural networks in the case of the greenhouse system is a combination of other alternatives: mechanistic models $[4,5]$. Thus, either technical or artificial intelligence as systems based on neural networks and fuzzy logic (neural-fuzzy system) would be extended and promising future applications.

\subsection{Distributed fluid dynamics models}

CFD techniques treat the values of the dependent variables as primary unknowns in a finite number of points, then a set of algebraic equations derived from the fundamental equations applied to the domain are solved through predefined algorithms. Three fundamental physical principles supporting the known Navier-Stokes equations are mass, momentum, and energy conservation. These are obtained for balance on a defined volume control, as given by Eq. (4)

$$
\frac{\partial(\rho \phi)}{\partial t}+\nabla \cdot(\rho \bar{u} \phi)=\nabla \cdot(\Gamma \nabla \phi)+S_{\varnothing}
$$

where the four terms correspond to transient, convection, diffusion, and source, respectively. They represent the variable $\varnothing$ depending on mass, velocity, temperature, and chemical species 
distributed in all locations in a given time $(\varphi=\varphi(x, y, z, t))$, in a Cartesian system with Euler viewpoint description. $\rho$ denotes density $\left(\mathrm{kg} \mathrm{m}^{-3}\right) ; t$ is time (s); $\nabla$ is the gradient operator; $\phi$ denotes the state variable like air temperature $(\mathrm{K})$, ammonia $\left(\mathrm{NH}_{3}\right)$, and mass of fraction $(\mathrm{kg}$ $\left.\mathrm{kg}^{-1}\right) ; u$ is the wind speed $\left(\mathrm{m} \mathrm{s}^{-1}\right) ; \Gamma$ is the diffusion coefficient $\left(\mathrm{m}^{2} \mathrm{~s}^{-1}\right)$; and $S_{\varphi}$ is the source term which represents the variation of the amount of material transported.

The computational studies of flow and heat transfer are mainly based on the solution of these equations together with the particular initial and boundary conditions that fulfill the problem. Commercial software regarding fluid dynamics have been designed with a user-friendly environment. To minimize the possibility of erroneously run away the desirable results by the numerical model is recommended to use prototype problems (benchmarks) $[6,7,8]$.

\subsubsection{Processes representation}

Since the atmosphere inside the greenhouse is influenced by local environmental variables, roofing material, irrigation systems, crop type, among others, a model based on computational fluid dynamics is able to represent these variables [9]. A simulation of wind distribution using CFD in a greenhouse is shown in Figure 1.

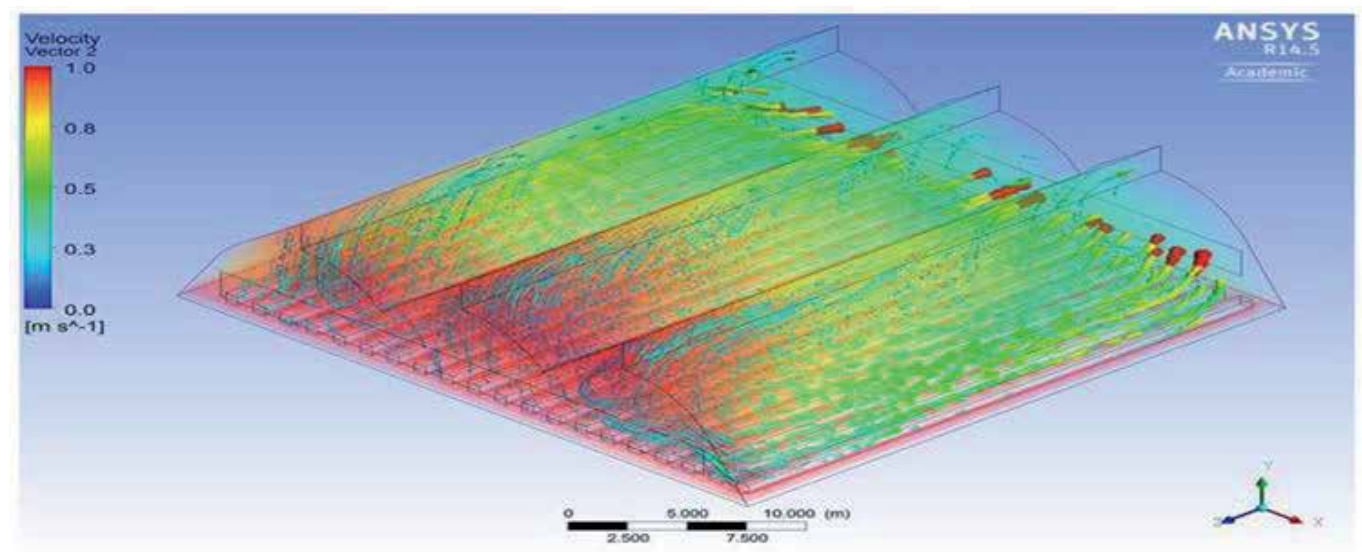

Figure 1. Climate conditions in a greenhouse by using CFD

\section{Computational fluid dynamics process simulation in a greenhouse}

The CFD is based on the Navier-Stokes equations, expressed as a solution of partial differential equations for mass and momentum balances. Their deduction is typically extended through heat and mass balance contained in a control volume. Such equations can be expressed in a generalized form as Eq. 4 [10]. Based on the three basics principles of conservation, mass, momentum, and energy, they can be represented in a conservative way 


\section{Continuity}

$$
\frac{\partial \rho}{\partial t}+\nabla \cdot(\rho \mathbf{V})=0
$$

Equation 5 is valid for any infinitesimal element, where $\varrho$ is the density of fluid $\left(\mathrm{kg} \mathrm{cm}^{-3}\right)$ and $\nabla \cdot \mathbf{V}$ represents the rate of change of volume, and $\frac{\partial \rho}{\partial t}$ is the density change with respect to time and $\mathbf{V}$ the flow velocity.

Momentum and energy equations are expressed for each component in Cartesian coordinates.

\section{Momentum}

X component:

$$
\frac{\partial(u)}{\partial t}+\nabla \cdot(\rho u \mathbf{V})=-\frac{\partial p}{\partial x}+\frac{\partial \tau_{x x}}{\partial x}+\frac{\partial \tau_{y x}}{\partial y}+\frac{\partial \tau_{z x}}{\partial z}+\rho f_{x}
$$

Y component:

$$
\frac{\partial(v)}{\partial t}+\nabla \cdot(\rho v \mathbf{V})=-\frac{\partial p}{\partial y}+\frac{\partial \tau_{x y}}{\partial x}+\frac{\partial \tau_{y y}}{\partial y}+\frac{\partial \tau_{z y}}{\partial z}+\rho f_{y}
$$

Z component:

$$
\frac{\partial(w)}{\partial t}+\nabla \cdot(\rho w \mathbf{V})=-\frac{\partial p}{\partial z}+\frac{\partial \tau_{x z}}{\partial x}+\frac{\partial \tau_{y z}}{\partial y}+\frac{\partial \tau_{z z}}{\partial z}+\rho f_{z}
$$

\section{Energy}

$$
\begin{aligned}
& \frac{\partial}{\partial t}\left[\rho\left(e+\frac{V^{2}}{2}\right)\right]+\nabla \cdot\left[\rho\left(e+\frac{V^{2}}{2}\right) \mathbf{V}\right]= \\
& =\rho q+\frac{\partial}{\partial x}\left(k \frac{\partial T}{\partial x}\right)+\frac{\partial}{\partial y}\left(k \frac{\partial T}{\partial y}\right)+\frac{\partial}{\partial z}\left(k \frac{\partial T}{\partial z}\right)- \\
& -\frac{\partial(u p)}{\partial x}-\frac{\partial(v p)}{\partial y}-\frac{\partial(w p)}{\partial z}+\frac{\partial\left(u \tau_{x x}\right)}{\partial x}+ \\
& +\frac{\partial\left(u \tau_{y x}\right)}{\partial y}+\frac{\partial\left(u \tau_{z x}\right)}{\partial z}+\frac{\partial\left(v \tau_{x y}\right)}{\partial x}+\frac{\partial\left(v \tau_{y y}\right)}{\partial y}+ \\
& +\frac{\partial\left(v \tau_{z y}\right)}{\partial z}+\frac{\partial\left(w \tau_{x z}\right)}{\partial x}+\frac{\partial\left(w \tau_{y z}\right)}{\partial y}+\frac{\partial\left(w \tau_{z z}\right)}{\partial z}+\rho \mathbf{f} \cdot \mathbf{V}
\end{aligned}
$$


where $\mathrm{q}$ is the rate of heat volumetric flow $\left(\mathrm{kJ} / \mathrm{m}^{3} \mathrm{~s}\right), \mathrm{k}$ is the thermal conductivity $\left(\mathrm{W} /\left(\mathrm{m}{ }^{\circ} \mathrm{C}\right)\right)$, Q is the fluid density $\left(\mathrm{kg} / \mathrm{m}^{3}\right)$, e is the internal energy of the fluid element due to random molecular motion per unit mass $(\mathrm{J}), \mathrm{V}^{2} / 2$ is the kinetic energy per unit mass $\left(\mathrm{m}^{2} / \mathrm{s}^{2}\right)$; this is due to translational movement of the fluid element, $\mathrm{T}$ is the fluid temperature $\left({ }^{\circ} \mathrm{C}\right), \mathrm{f}$ is the force $(\mathrm{N})$, and $\tau$ is the Reynolds stress, which represents the turbulent fluctuations of the fluid.

The equation for the internal energy e can be expressed as the static enthalpy $h$. Indeed, the total enthalpy is given by $\mathrm{h}=\mathrm{h}+\mathrm{V}^{2} / 2$.

The simulation is performed assuming steady-state conditions. Additionally, the greenhouse airflow patterns are assumed to be turbulent and the $k-\varepsilon$ model is applied to solve for the kinetic energy $(k)$ and the viscous dissipation rate of turbulent energy $(\varepsilon)$. Several researchers have shown that this model makes good representation of the turbulent nature of fluid flow within controlled environments [11, 12,13,14] and it is one of the most commonly used [15].

Because low wind speeds prevailed in the experimental site through the year, the average wind speed and gust speed computed from the measured values were 1.89 and $4.34 \mathrm{~m} \mathrm{~s}^{-1}$, respectively. Free and mixed convection may drive air exchange, instead of forced convection. Similar convection regimes were simulated in this study, as in previous studies performed on the same location [16].

\subsection{Problem formulation}

The simulation of physical and biological processes within the greenhouses is the challenge of the century from the numerical point of view. Although the theoretical basis had been established over the last centuries, advances in scalar or visualization representation were not given until this decade.

It is common to simulate single physical and biological processes, but is still pending models which combine, for example, the processes of photosynthesis and respiration, in order to improve the production of greenhouse crops.

\subsubsection{Climate modeling}

The analysis by CFD involving heat and mass transfer following the numerical solution of the fundamental equations of mass, energy, and momentum conservation, can deal with transient and distributed solutions, illustrating in space ( 2 or 3 dimensions) and time the variables of the climate and atmosphere, simultaneously.

Air movement will be based on a temperature gradient and consequently phenomenon of convection mass transport. Two models are essential for the analysis of this case; the flotation model for natural convection accounts for the variation of density as a function of temperature. Boussinesq model is used for suitable results and is given by Eq. (10) [17].

$$
\rho=\rho_{i} 1-\beta\left(T-T_{i}\right)
$$


where $\rho$ is the density $\left(\mathrm{kg} \mathrm{m}^{-3}\right), \beta$ is the coefficient of thermal expansion $\left(\mathrm{K}^{-1}\right), T$ is the air temperature $(\mathrm{K})$, and the subscript $i$ means inside.

Finally, air movement assumes a mixture of liquid, steam, and nonconsumable gas, the standard equation that governs the mixture model and the mixing turbulence model flow for vapor mass fraction (f) can be written as Eq. (11) [18].

$$
\frac{\partial}{\partial \varepsilon}(\rho f)+\nabla\left(\rho \vec{v}_{v} f\right)=\nabla(\gamma \nabla f)+R_{e}-R_{c}
$$

Beginning from this statement, the air movement has been exposed. For instance, Figures 2, 3, and 4 show the dynamics of the air into the greenhouse, under different frames, cenital (Figure 2), tunnel (Figure 3), and screen house (Figure 4).

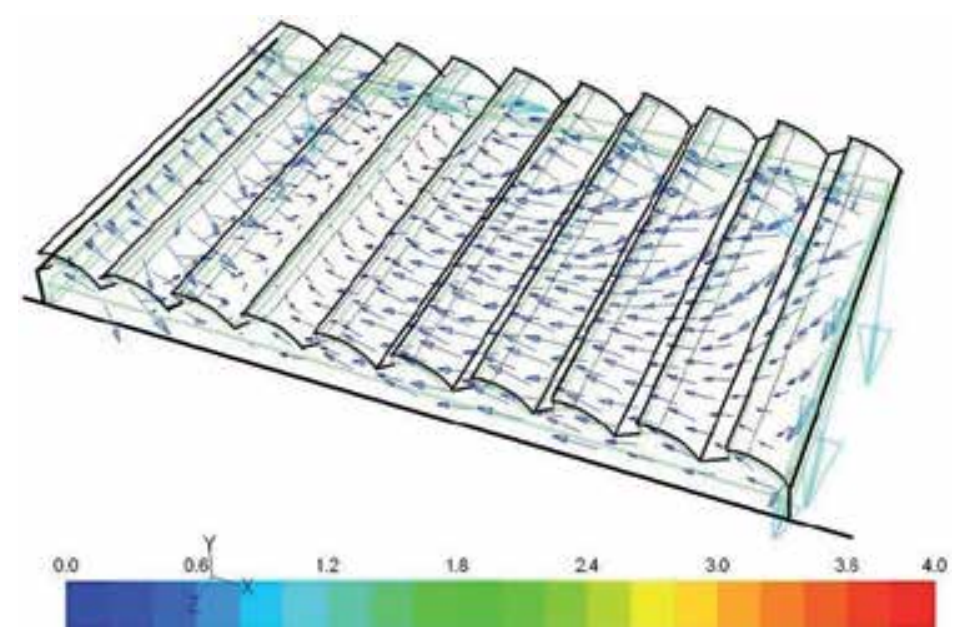

Figure 2. Wind behavior in a multi-spam (roof windows) frame

\subsubsection{Mechanical ventilation}

The mechanical ventilation is less used than natural ventilation, therefore investigation about this is scanty. For instance, the American Society of Agricultural Engineers (ASAE) provides the rules for the design of the mechanical ventilation system [19]; however, [20] there are limitations to these rules, such as the difficulty to incorporate approximate values for the transpiration coefficient in the calculus of the ventilation system.

Other studies like [21] and [22], show that the ventilator allows more control on the temperature in the greenhouse than the passive ventilation [23], and found a vertical gradient of temperature and humidity in the air much more homogeneous with mechanical ventilation. However, in other studies like [19], it was observed that even with mechanical ventilation there can be a notable lack of thermal and humidity homogeneity. Since they measured major 


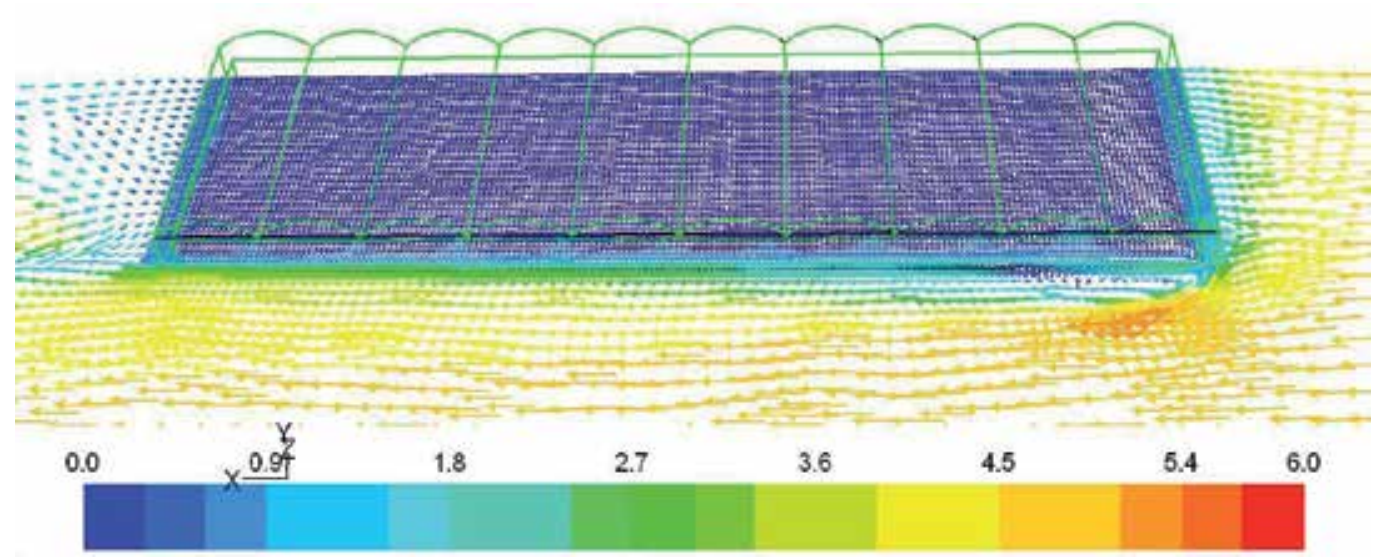

Figure 3. Wind velocity $\left(\mathrm{m} \mathrm{s}^{-1}\right)$ in a tunnel frame

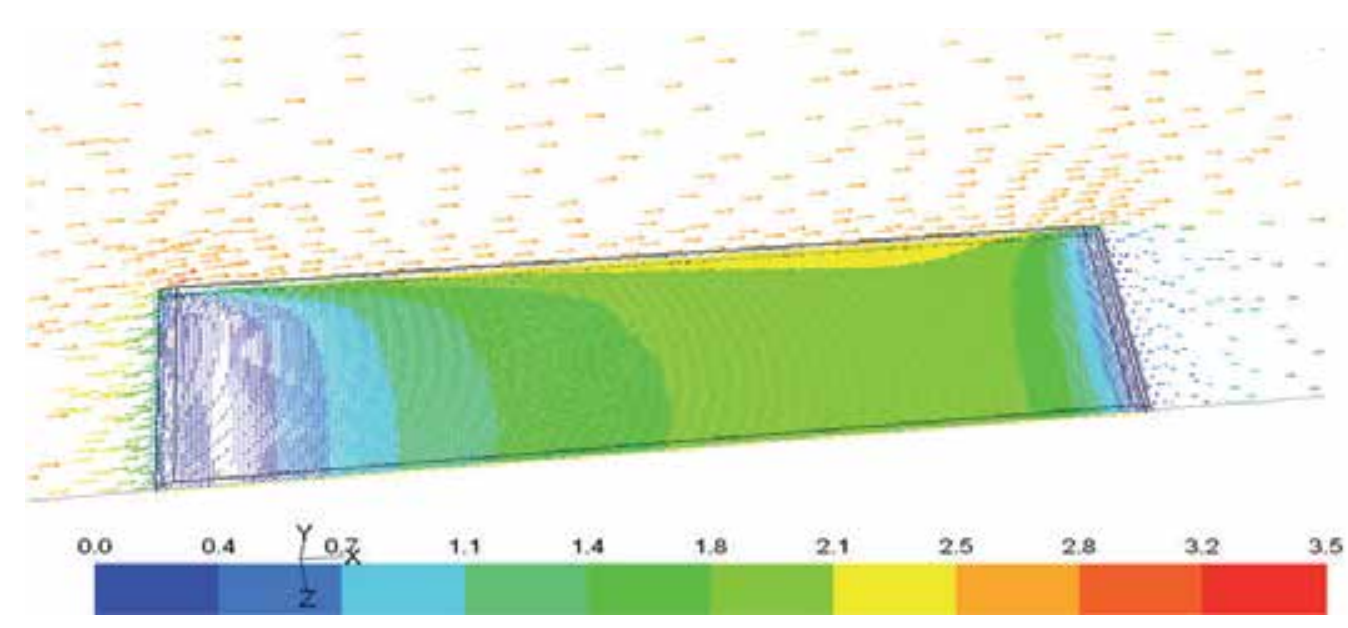

Figure 4. Wind velocity $\left(\mathrm{m} \mathrm{s}^{-1}\right)$ and distribution in a screen house frame

temperatures in the high part of the crop for some time, the homogeneity can also be improved if roof windows are lightly open, especially when the greenhouse is big [24]. Both, mechanical and natural ventilation are processes with a high degree of difficulty in the analysis. So the investigation about the combination between natural and mechanical ventilation is very incipient and limited.

Nowadays, Computational Fluid Dynamics (CFD) is a strong tool for analysis, because it allows to study in detail the ventilation process and climatic variables inside of the greenhouse $[24,25]$. Questions on the management of the system of ventilation to support sufficient traffic of the air and to achieve acceptable levels of heat transmission and mass between crop and the air can be solved by means of the application of this tool [13, 25-28]. An example of mechanical ventilation in a greenhouse is shown in Figure 5. 


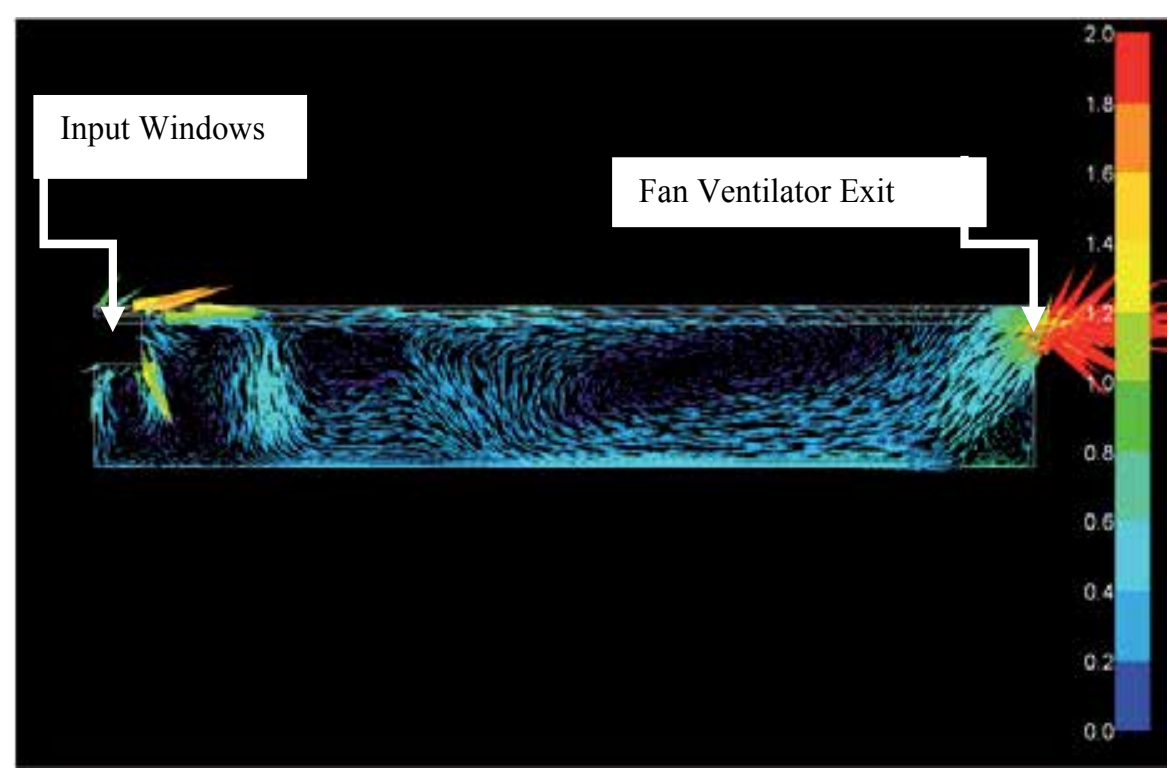

Figure 5. Wind vectors as a result of mechanical ventilation system (colored by magnitude)

\subsubsection{Heating system}

Greenhouse heating is one of the factors with a significant impact on crop production. From the environmental and economic points of view, the use of fossil fuels in agricultural production is costly; however, in regions with cold winters, the use of heating systems is essential to achieve sustainable production. A method to prevent the temperature going below the minimum threshold for crop production could be based on the forces of convection.

The heating source can be of various types such as collector walls or heating systems, based on water or gas driven through pipes. Heating pipes are effective devices used to keep the greenhouse warm and are implemented in many of the cold climate greenhouses to maintain a targeted temperature [29]. Their principles for heat transfer are convection and radiation; however there is a need to find ways to increase their efficiency. The position of the pipes in the greenhouse and the power of the heating system influence the spatial distribution of temperature and flow patterns due to convective forces.

Reference [29] reported that the best place to locate the pipes is at a half crop height, with the tubes near to the leaves. Other configurations have been studied by several researchers [30, 31], which highlighted the influence of the heating system on the cultivation in terms of convection and radiation. As a result of these studies, the advantages of placing a pipe heating system at the bottom of the crop without affecting the evapotranspiration from plants were discovered. Reference [32], reported that this type of heating system favors the removal of moisture. Humidity, as well as other factors, such as condensation [33] and cooling [34], especially in closed greenhouses [9], have been analyzed by computational fluid dynamics (CFD). 
From the numerical point of view, reference [35] performed an experimental validation of a greenhouse model based on CFD to analyze its air thermal gradients. They found that the largest temperature differences occur in the zones close to the ground and under the roof. Conversely, air temperatures inside the greenhouse were more uniform and had a homogeneous distribution. Similarly, Tadj et al. [36] used ANSYS CFX to analyze the airflow and temperature patterns under three scenarios simulating several pipe locations and a tomato crop. They observed the same behavior of the air and temperature patterns, which consisted of strong thermal gradients near the floor and ceiling, and a homogeneous distribution in the rest of the greenhouse.

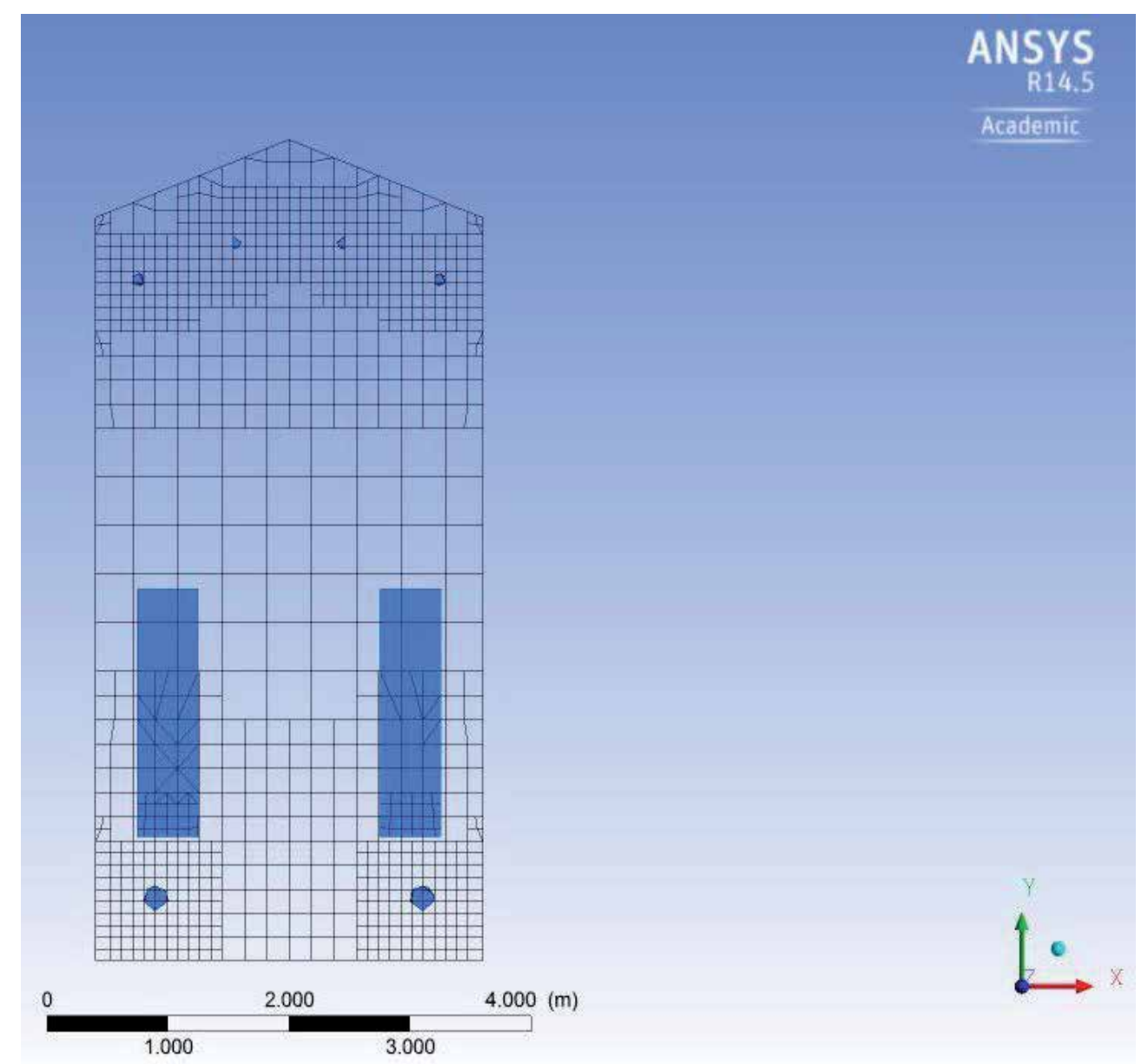

Figure 6. Meshing in a cabinet with heating system by pipes (low and upper)

In this chapter the feasibility of the natural convection phenomena as an energy transference process has been analyzed through simulations, produced in a Venlo type greenhouse with the presence of a fully developed tomato crop. Convection forces were assumed to be produced 
by the energy stored naturally in the soil mainly due to solar radiation and the energy produced from a pipe heating system (figure 6). The crop was simulated with the "single leaf" approach [37] by considering the plant canopy as a porous zone. CFD simulations were performed for the analysis considering the effects of an adiabatic wall temperature and the external environment. External temperature was given as a boundary condition for the plastic cover (figure 7). Results show the behavior of the greenhouse environment when it is driven by natural convection and assisted by a heating system of this kind.
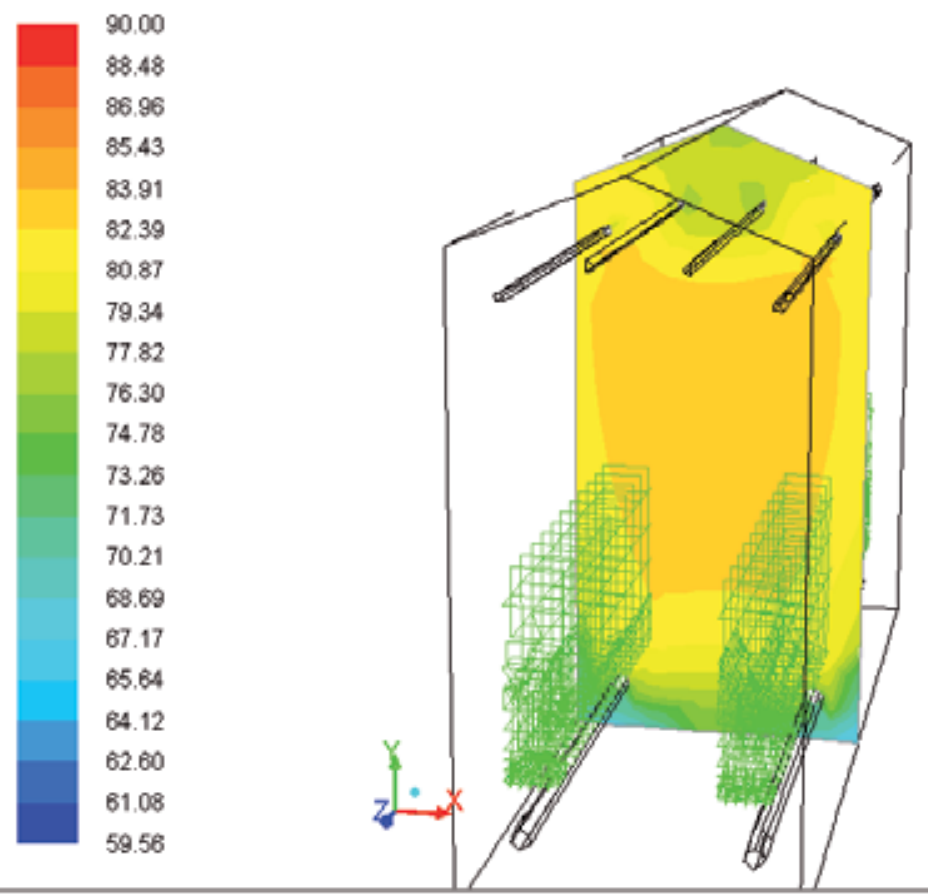

Figure 7. Contour of the Relative Humidity (\%) in the center of cabinet

\subsubsection{Mass diffusion within turbulent flows}

Air is assumed as a mixture of liquid, vapor, and non-consumable gases. In this study, the species transport model available in FLUENT is possibly used to simulate the mass transport of different gases, such as $\mathrm{CO}_{2}$, nitrogen, ammonia, etc., beginning from the diffusion flux of species $i$, which arises due to gradients of concentration and temperature. Such species model uses the dilute approximation (Ficks's law) to model mass diffusion. For turbulent flows, mass diffusion can be written as in Eq. 12 [18] 


$$
\bar{J}_{i}=\rho D_{i, m} \nabla Y_{i}-D_{T, i} \frac{\nabla T}{T}
$$

where $J_{i}\left(\mathrm{~m}^{2} / \mathrm{s}\right)$ is the diffusion flux of species $i$ (ammonia), $\rho$ is the density of the mixture $(\mathrm{kg} /$ $\left.\mathrm{m}^{3}\right), D_{i, m}$ is the mass diffusion coefficient for specie $i$ in the mixture $m\left(\mathrm{~m}^{2} / \mathrm{s}\right), D_{T, i}$ is the turbulent diffusion coefficient $\left(\mathrm{m}^{2} \mathrm{~s}^{-1}\right), Y i$ is the mass fraction of specie $i$, and $T$ is the temperature of the flow $(\mathrm{K})$.

To achieve the management of environment in the greenhouse, is fundamental the simulation, to know which scenario would be the best to obtain a better production for the specific case. That is why, the gasses simulation are necessary. One specific case is the animal farm. Rabbits and hens are the most representative cases of toxic gasses production. On the other hand, in a greenhouse, the $\mathrm{CO}_{2}$ production or extinction is a critical point in the crop production process.
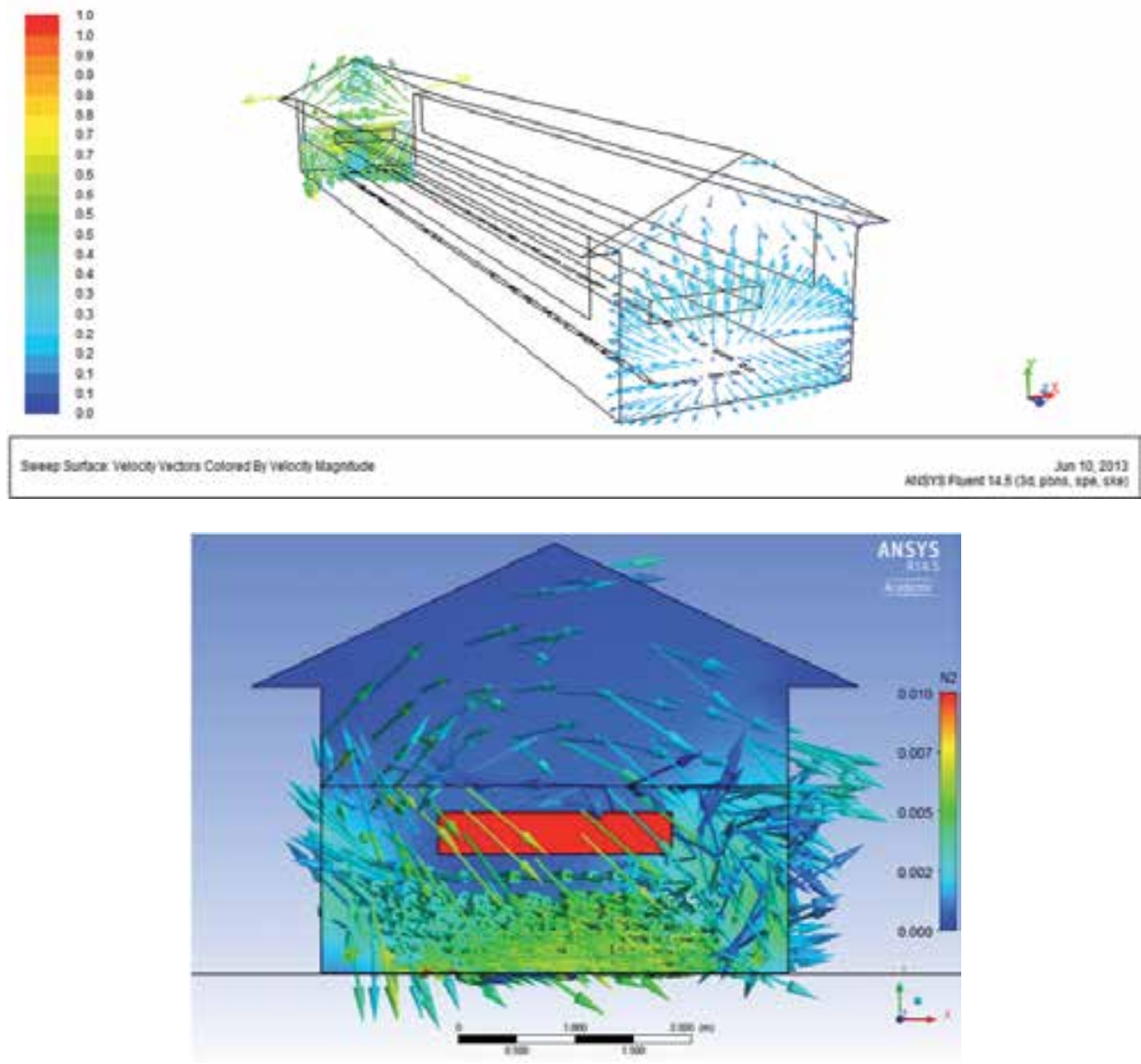

Figure 8. Wind velocity (up) and nitrogen distribution (dawn) in a domestic barn 
As a result of the application of the diffusion equations and species model to simulate some gasses, specific simulations are shown in Figures 8 and 9.

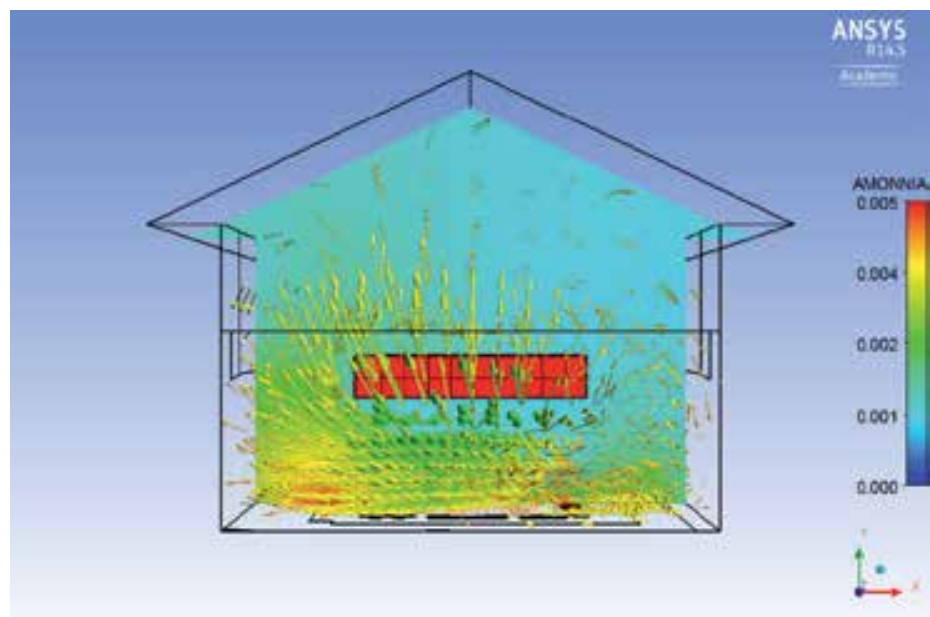

Figure 9. Ammonia representation as a result of natural ventilation system

\subsubsection{Distribution of ammonia}

Wind direction is the most important factor to exchange air in a semi-closed farm. The concentration of ammonia is greater near the ground because it is where the ammonia is produced (source) and because air renovation is limited due to the vents being located at an elevated position (Figure 9).

Figures 9, 10,11, and 12 represent ammonia distributions in a structure with rabbit production. If the wind is simulated parallel to the vents, the incoming air produced a lower concentration gradient at the zone beneath the cages (Figure 10). Lower concentration of ammonia results around the rabbit cages, even with the small ventilation rate produced. Thus, ammonia was more uniformly distributed when the wind direction was parallel.

Even though the air ventilation rate was much higher for the case when the wind direction was perpendicular to the vents, a notorious accumulation of ammonia in the area underneath the cages was produced (Figure 9). Ventilation rates increased at the vents level, but air circulation near the ground was limited. Therefore, the concentration of ammonia in the lower zone of the barn was higher than the concentration in the cages area and above. This was probably attributed to a limited air circulation within that zone.

If ammonia gets highly concentrated underneath, as it can be observed in Figure 10 for the scenario II, it represents a hazardous condition. The ammonia accumulated will eventually arise due to a flotation effect when high temperatures are present and that condition may be dangerous for the rabbits. 
The ventilation system design is the restricted air circulation observed underneath the cages on the simulation. Thus, having both vent openings at an elevated position from the ground level may not be effective to promote enough ventilation rates and lower the ammonia concentrations sufficiently, especially beneath the rabbit cages. That could be alleviated by lowering the height of the vents in the rabbit barn. Therefore, a modification on the vents position was proposed and simulated.

The modification on the ventilation system of the rabbit barn consisted of having a difference in height between the vent inlet and the vent outlet (Figure 10). The vent inlet was at $0.2 \mathrm{~m}$ above the ground level and ran continuously $22 \mathrm{~m}$ along the barn. The position of the vent outlet remained as in the original design, at a height of $1.2 \mathrm{~m}$ from the ground level.

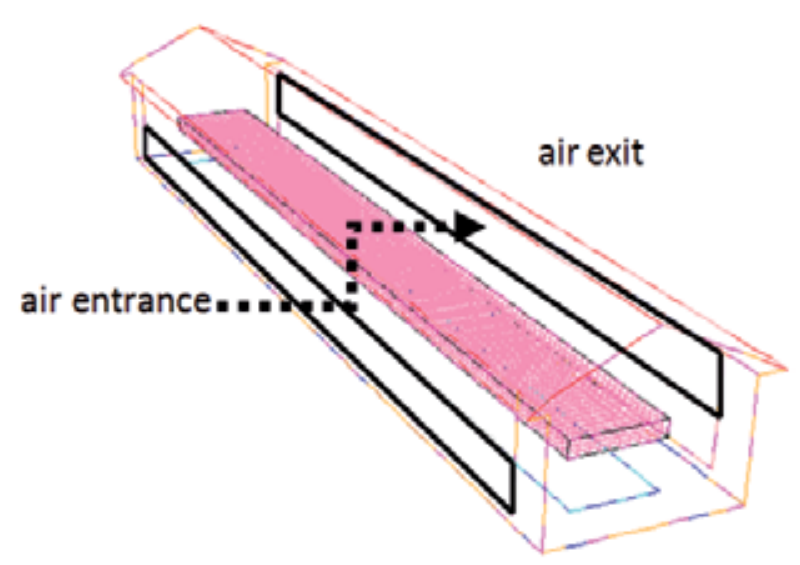

Figure 10. Design modification on the vent configuration of a barn. Vent inlet and vent outlet at a height of $0.2 \mathrm{~m}$ and $1.2 \mathrm{~m}$ above the ground, respectively

Air temperature and ammonia mass fraction on the vertical profile at the center of the barn are shown in Figures 11 and 12. Figure 11 shows the case when the wind is parallel and Figure 12 , when the wind is perpendicular to the barn. For presentation purposes, the height of the barn is shown in the Y-axis (dependent variable). Results show an improvement on the vertical distribution of air temperature and the ammonia mass fraction with the inlet vent located at $0.2 \mathrm{~m}$ of height (Figure 11). Air circulation was particularly improved in the area underneath the rabbit cages, resulting in a reduction of ammonia concentration. A similar result can be seen in Figure 12 (parallel wind direction) where the thermal vertical gradient improved significantly and a reduction of ammonia concentration was achieved in the zone below the rabbit cages.

\subsubsection{Theoretical approach of crop}

An approach in the analysis of greenhouse ventilation consists in establishing a reliable methodology that describes the interactions that are taking place in the interface porous matrix-fluid. 


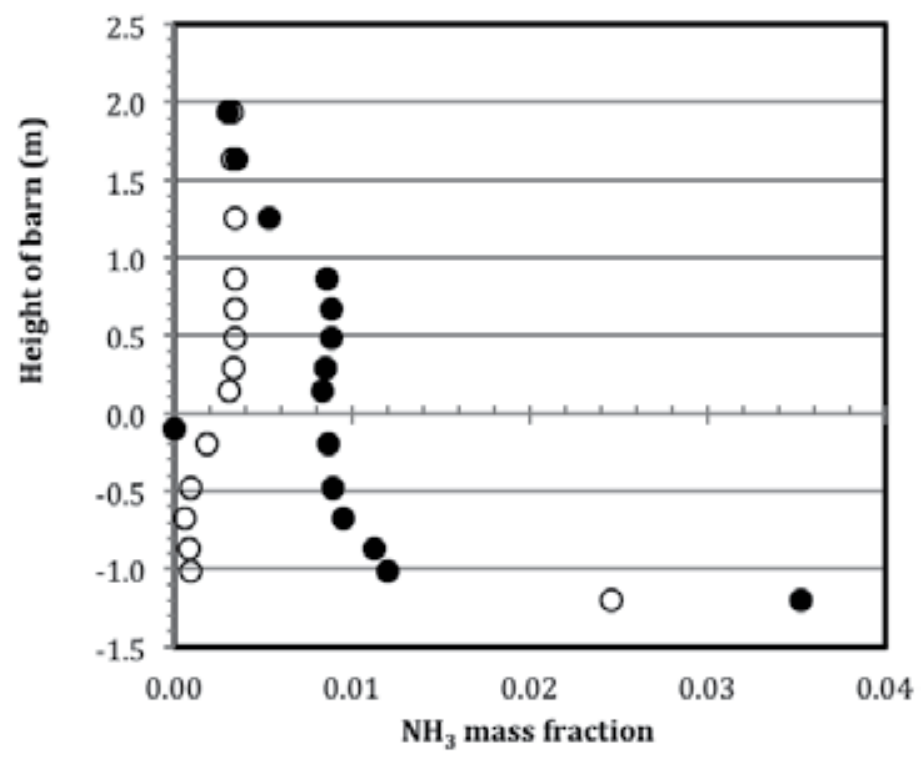

Figure 11. Ammonia distribution (mass fraction) wind direction parallel

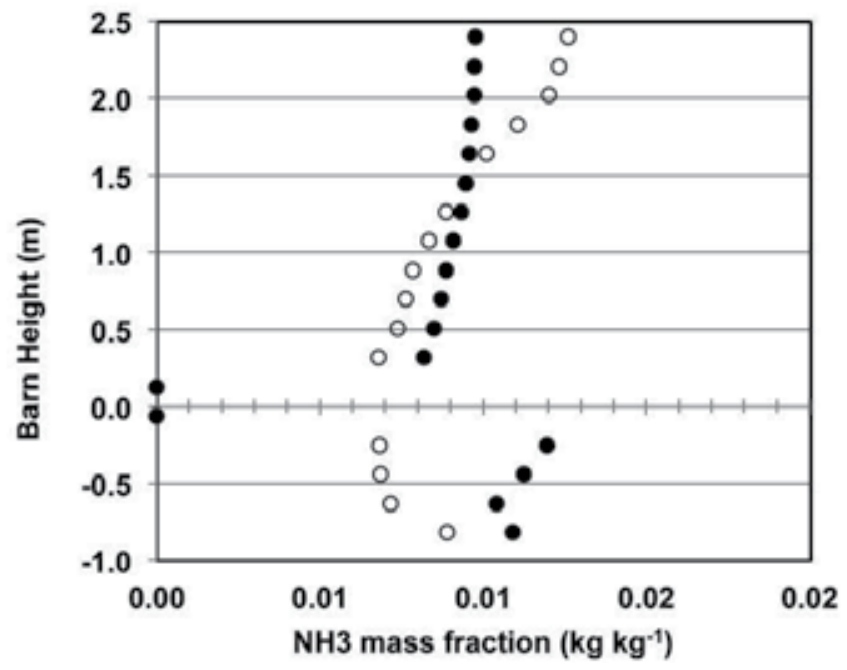

Figure 12. Ammonia distribution (mass fraction) wind direction orthogonal

The flow characteristics in a permeable material can be described in terms of permeability and porosity [38]. If a specific volume is considered, the porosity is represented by the space for which the fluid flows in relation with the total space contained in this volume. The porosity is 0 for zero flow and when it does not exist flow restrictions, the porosity will be 1 . The permeability is defined as the ability of the material to flow across itself, analogous when a conduit is totally opened the permeability tends to infinite. 
One of the first works to model the flow was published by [39] and later was used by Miguel et al. [38], who indicated that Eq. 13 can be used for analysis of one-dimensional mass transfer across a permeable material

$$
\frac{\partial}{\varepsilon} \frac{\partial u}{\partial t}+\frac{\rho}{\varepsilon^{2}} u \frac{\partial u}{\partial x}=-\frac{\partial p}{\partial x}-\frac{u}{K} u-\rho \frac{Y}{\sqrt{K}} u|u|+\frac{\mu}{\varepsilon} \frac{\partial^{2} u}{\partial x^{2}}
$$

where $u\left(u_{i} \varepsilon\right)$ is the superficial fluid speed $(\mathrm{m} / \mathrm{s}), u_{i}$ is the speed across the material $(\mathrm{m} / \mathrm{s}), \varepsilon$ is the porosity $\left(\mathrm{m}^{2} / \mathrm{m}^{2}\right)$, $\mathrm{Q}$ is the density of the fluid $\left(\mathrm{kg} / \mathrm{m}^{3}\right), \mathrm{P}$ is the pressure $(\mathrm{Pa}), \mu$ is the dynamic viscosity $(\mathrm{kg} / \mathrm{m} \cdot \mathrm{s}), \mathrm{Y}$ is the factor of inertia, $\mathrm{x}$ is the flow direction $(\mathrm{m})$, and $\mathrm{K}$ is the media permeability $(1 / \mathrm{m})$.

Equation 14 is a general equation in explicit form that indicates how the flow speed is related to the pressure gradient by the force to the viscous resistance

$$
\frac{\rho}{\varepsilon^{2}} u \frac{\partial u}{\partial x}
$$

The effect of inertia due to the pore is included in the following relation

$$
\rho \frac{Y}{\sqrt{K}} u|u|
$$

Equation 15 represents the flow of high pressure inertial loss. The force of viscous resistance due to the transfer at the moment in the interface matrix-fluid is included as Eq. 16

$$
\frac{\mu}{K} u
$$

And the resistance for viscosity of the flow of the fluid as Eq. 17

$$
\frac{\mu}{\varepsilon} \frac{\partial^{2} u}{\partial x^{2}}
$$

The equation of fluid movement across a porous media can be derived from this equation.

\subsubsection{Crops (porous zone)}

To study the effects of crop canopy in the airflow inside a greenhouse, Haxaire [40] made an experiment in a wind tunnel to determine the speed loss (drag effect) due to the presence of 
plants. He also analyzed the effect on the pressure drop (Dp) and the density of foliar area (L) due to changes in the air speed. The determination of the dragging coefficient has allowed new incursions in the study of the fluid dynamics; such studies have been conducted introducing this coefficient [41-44, 26].

The analysis and calculation of the effect of crops can be realized adding the term source into the Navier-Stokes Equation (Eq. 18):

$$
\frac{\partial(\rho \varnothing)}{\partial t}+\nabla(\partial(\bar{u} \varnothing))=\nabla(\Gamma \nabla \varnothing)+S_{\varnothing}
$$

The source term $S_{\varnothing}$ contains the variable of interest, which represents the moment consumption due to the effect of dragging (drag effect) of the crop. This friction force can be expressed as the unit of volume of coverage using the Wilson formula [45] (Eq. 19).

$$
S_{\varnothing}=-L C_{D} v^{2}
$$

where $v$ is the speed of the air $(\mathrm{m} / \mathrm{s})$, L refers to the canopy density $\left(\mathrm{m}^{2} / \mathrm{m}^{3}\right)$, and $C_{\mathrm{D}}$ is the friction coefficient (drag coefficient), which [40] experimentally established as 0.32 . In order to include the effect of dragging or friction (drag effect) proportionally to the density of foliate area, the crop (cultures) was considered as porous media and it was described by Darcy-Forchheimer in [47], as shown in Eq. 20.

$$
S_{\varnothing}=-\left[\frac{\mu}{K} v\right]+\rho \frac{C_{f}}{K} v^{2}
$$

where $C_{\mathrm{f}}=$ is the loss coefficient.

In greenhouse ventilation studies, the wind speed is such that the quadratic term of Eq. 20 dominates the linear term. This term can be neglected due to the small lost coefficient in the linear moment equation. The intrinsic permeability K can be deduced combining Eq. 19 and Eq. 20 by the following relation (Eq. 21)

$$
\frac{C_{f}}{K}=L C_{D}
$$

Dragging coefficients were estimated using experimental data for each crop as shown in Table 1, by applying Eq. 19 and Eq. 20. The flow reduction due to crop was simulated in the source term $\left(S_{\varnothing}\right)$ in Eq. 19. 


\begin{tabular}{lccc}
\hline Crop & LAD $\left(\mathrm{m}^{2} \mathbf{m}^{-3}\right)$ & Cd & S $\phi$ \\
\hline Tomato & 5.6 & 0.26 & 2.9 \\
Pepper & 5.8 & 0.23 & 2.7 \\
Egg Plant & 3.7 & 0.23 & 1.7 \\
Beans & 3.0 & 0.22 & 1.6 \\
\hline
\end{tabular}

Table 1. Characterization of four crops with different leave shape (Adapted from [46]).

The shape of the leaves determines air flow. Different crop shapes are shown in Figure 13.
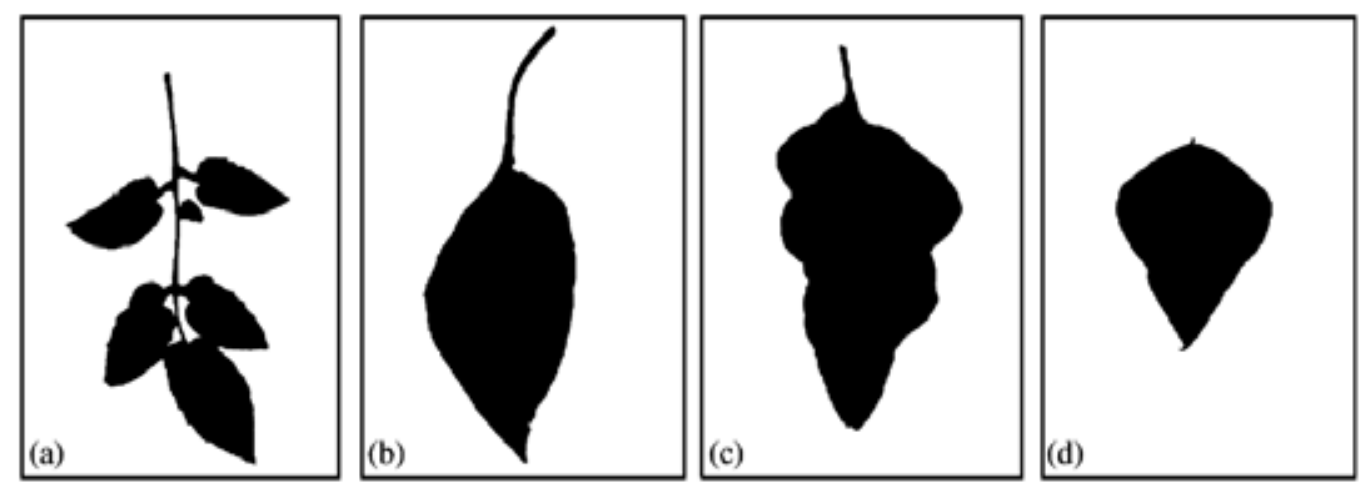

Figure 13. Morphology of the leaves of a) Tomato, b) Pepper, c) Egg plant, and c) Beans. Adapted from [46]

The unload coefficient is the factor that determines momentum loss according the transport equation; when insect mesh windows are used, the pressure drop is $80 \%$, with a variation of $8 \%$ depending on the mesh porosity [48]. This implies that air speeds inside the greenhouse usually are less than $1 \mathrm{~m} / \mathrm{s}$. The reduction is accentuated along the cropped area from the air inlet, depending on cropped length and in a staked crop.

In this case, the wind speed drop for two crops studies is shown in Figure 14. The results show that even if the leaves configuration is different, the pressure drop depends principally on canopy density. This can be attributed that differences in wind speed are not significant. In addition, we have the hypothesis that at the first contact with the crop (Figure 14), airflow modifies the angle inducing a leaves "alignment" that favor the flow and becomes constant and less restrictive. The presence of crop canopy represents a reduction in airflow, which increases as moved away from the wind-inlet window. In this study, an immediate drop was of $0.1 \mathrm{~m} / \mathrm{s}$, but as becomes stable, the speed variations are of $\pm 0.05 \mathrm{~m} / \mathrm{s}$ in $34 \mathrm{~m}$ of greenhouse length.

Due to presence of canopy, there is a speed reduction of $33 \%$ between the inlet and outlet windows (above) through crop canopy (Figure 15A). Inside the canopy, the wind speed is 


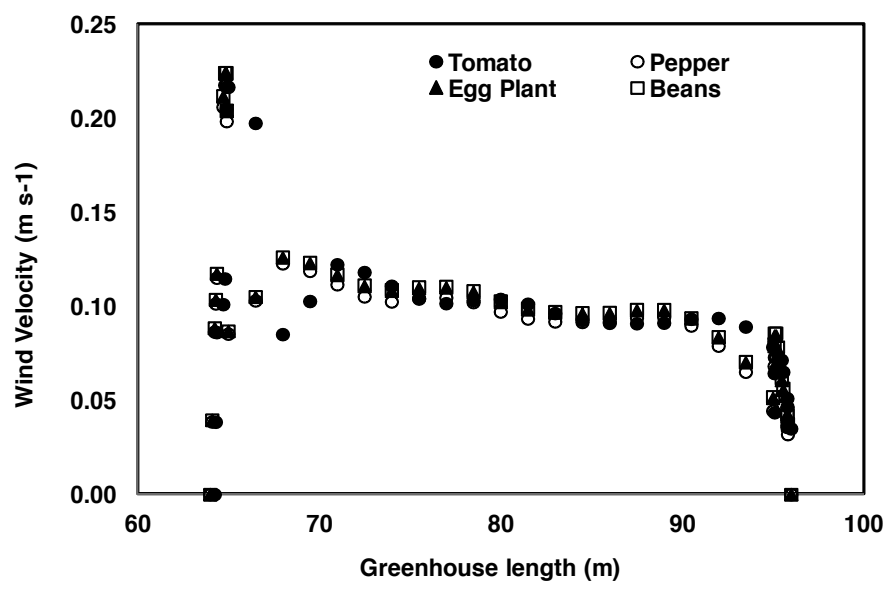

Figure 14. Longitudinal variation of the wind speed for four horticultural crops with different $\mathrm{Cd}$

about $0.1 \mathrm{~ms}^{-1}$, but above this, the increase is very small, up to $0.25 \%$; this can be very important for best placement of sensors to measure transpiration. This process is directly related to wind speed [49].

As a consequence of flow variation, the temperatures showed an increase toward the windows outlet. A $6 \mathrm{~K}$ increase was estimated (Figure 15B) with the particularity that this variable is dependent on the canopy height. This indicates the convective transport of the heat inside the greenhouse can be more homogeneous and independent from the air flow rate as it becomes stable.

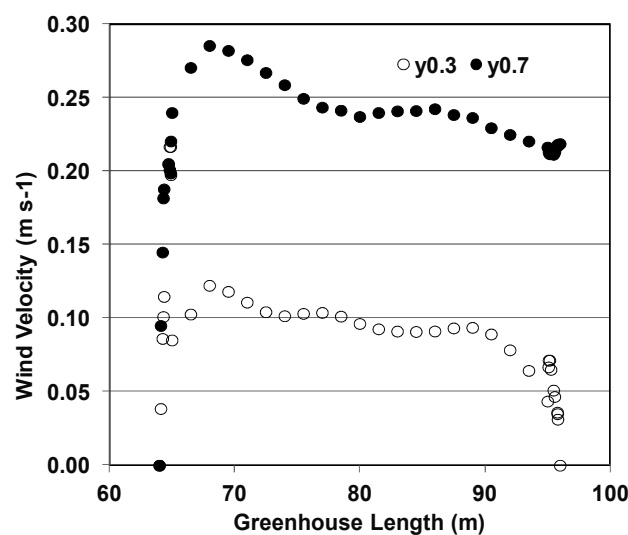

a)

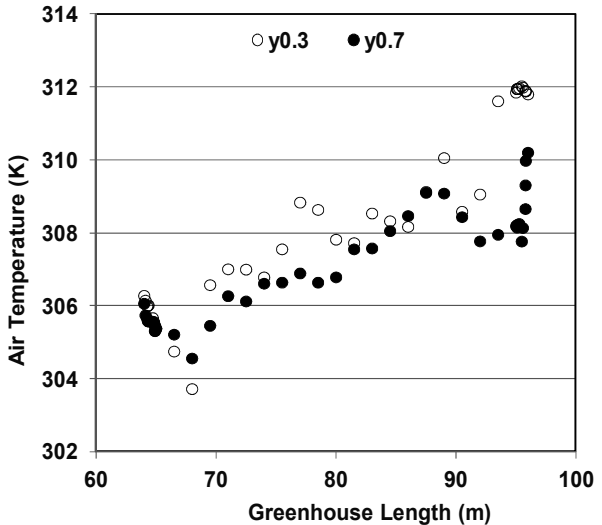

b)

Figure 15. Variation of the wind velocity (m s-1) (A) and temperature (K) (B) in the cropped area (tomato) along the greenhouse $(\mathrm{m})$ 
Schematically, Figure 16 shows the distribution of the speed vectors in the dominant wind direction, and spatial distribution of the temperature in the greenhouse ambient.

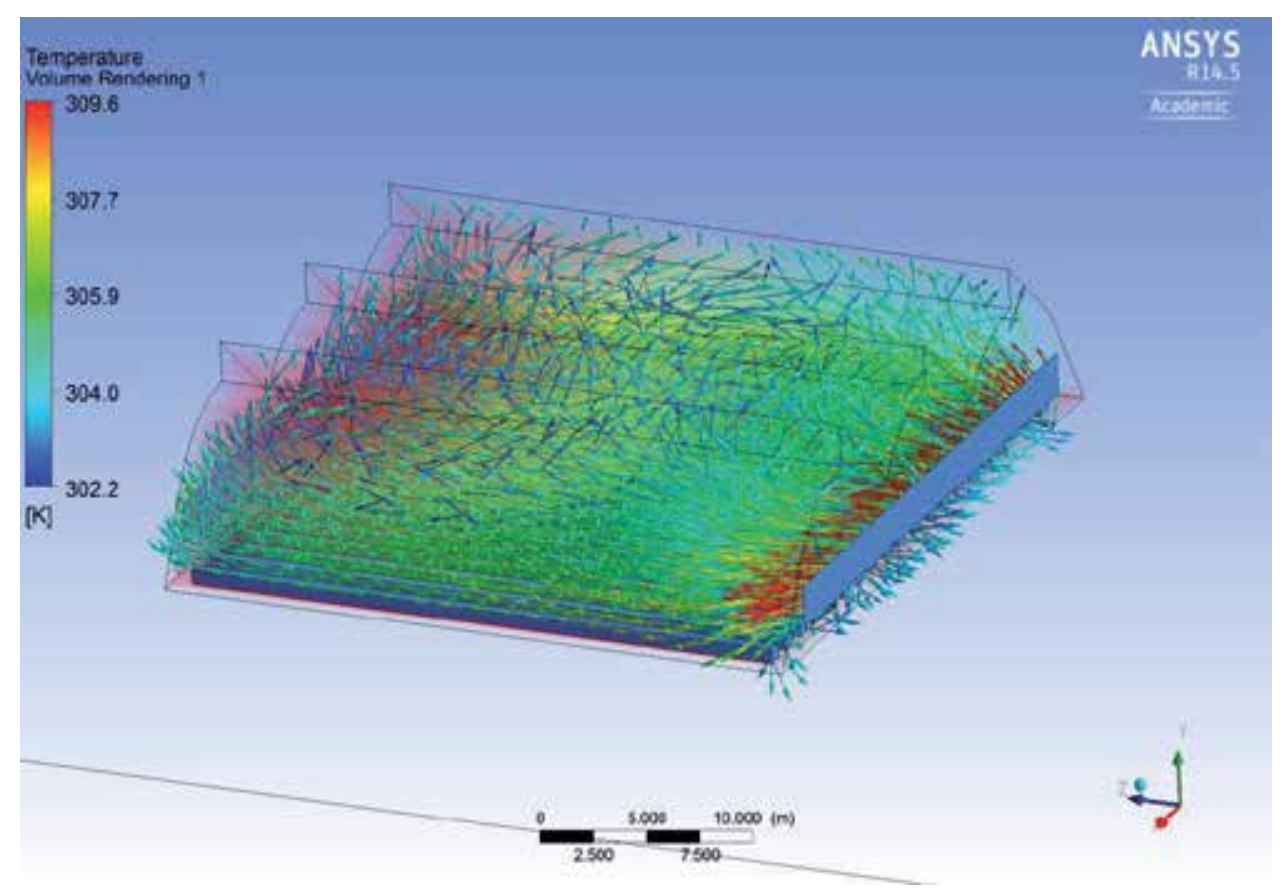

Figure 16. Spatial distribution of wind speed vectors $(\mathrm{m} / \mathrm{s})$ and temperature $(\mathrm{K})$

\section{Conclusions}

Most of the processes that occur in a greenhouse are related to mass transfer events (relative humidity, transpiration), momentum (velocity, species), and energy or exchange heat (convection, radiation) and consequently, the computational fluid dynamics is a robust tool for greenhouses and animal farm behavior simulation. The inclusion of culture and auxiliary climatic control systems are essential to the production process from an agricultural point of view. The results of this study indicate that once experimentally validated models, the use of these allows to know the spatial characteristics of the variables involved. From the mechanical point of view, the use of anti-insect screens and crop presence causes a drop of $80 \%$ in speed. A heating system for hot pipes under conditions of central Mexico is only necessary for specific periods before sunrise. A change in the position of the greenhouse windows in zigzag increases air movement and homogenized conditions inside, even if you keep the same ventilation ratio, but a variation in the porosity of the mesh has side effects in the air renewal ratio. The prospect of computational fluid dynamics applied to the protected agriculture, will focus on building 
a dynamic model with many variables to make efficient use of resources and reduce the use of fossil fuels in crop production.

\section{Author details}

Jorge Flores-Velázquez ${ }^{1 *}$, Abraham Rojano ${ }^{2}$, Adriana Rojas-Rishor ${ }^{3}$ and Waldo Ojeda Bustamante ${ }^{1}$

*Address all correspondence to: jorge_flores@tlaloc.imta.mx

1 Mexican Institute of Water Technology, Morelos, México

2 Autonomous University of Chapingo, Texcoco, México, México

3 University of Costa Rica, San José, Costa Rica

\section{References}

[1] Ljung L. System identification: theory for the user, 2nd ed. Prentice Hall, Linköping University, 1999, 375-380 p.

[2] Seginer I, Boulard T, Bailey B.J. Neural network models of the greenhouse climate. Journal of Agricultural Engineering Research. 1994; 53: 203-216.

[3] Boaventura J, Ruano A, Couto C. Identification of greenhouse climate dynamic models. In: Sixth international conference on computers in agriculture, Cancún, 1996, 161-171.

[4] Dayan J, Strassberg Y, Dayan E. Simulation and control of ventilation rates in greenhouses. Acta Horticulturae. 2001; 566: 67-74.

[5] Dayan J, Strassberg Y, Dayan E. The prediction of ventilation rates in greenhouses containing rose crops. Acta Horticulturae. 2002; 593: 55-62.

[6] Al-helal I. A computational fluid dynamics study of natural ventilation in arid region greenhouses. PhD thesis, Ohio State University, 1998.

[7] Romero-Gómez P, López-Cruz IL, Choi CY. Analysis of greenhouse natural ventilation under the environmental conditions of central Mexico. Transactions of the ASABE. 2008; 51: 1753-1761.

[8] Rico-García E, López-Cruz IL, Herrera-Ruiz G, Soto-Zarazúa GM, Castañeda-Miranda R. Effect of temperature on greenhouse natural ventilation under hot conditions: CFD simulations. Journal of Applied Sciences. 2008; 8: 4543-4551. 
[9] Flores-Velázquez J, De la Torre-Gea G, Rico-García E, López-Cruz IL, Rojano-Aguilar A. Applied to the greenhouse environment. In: Hyoung Woo Oh (ed) Applied computational fluid dynamics. InTech, Croatia, 2012, 37-62.

[10] Anderson JD. Computational fluid dynamics. University of Maryland: McGraw Hill, 1995, 37-80 p.

[11] Li W. Airflow and contaminants in a swine barn with recirculation assisted slot inlets. PhD thesis, University of Saskatchewan, 1997.

[12] Mistriotis A, Bot G.P., Picuno P, Scarascia-Mugnozza G. Analysis of the efficiency of greenhouse ventilation using computational fluid dynamics. Agriculture and Forest Meteorology. 1997; 85: 217-228.

[13] Norton T, Sun D, Grant J, Fallon R, Dodd V. Application of computational fluid dynamics (CFD) in the modeling and design of ventilation systems in the agricultural industry: A review. Bioresource Technology. 2007; 98: 2386-2414.

[14] Norton T, Grant J, Fallon R, Sun DW. Assessing the ventilation effectiveness of naturally ventilated livestock buildings under wind dominant conditions using computational fluid dynamics. Biosystems Engineering. 2009; 103: 78-99.

[15] Bjerg B, Cascone G, Lee IB, Bartzanas T, Norton T, Hong SW, Seo IH, Banhazi T, Liberati $P$, Marucci A, Zhang G. Modelling of ammonia emissions from naturally ventilated livestock buildings. Part 3: CFD modelling. Biosystems Engineering. 2013; 116: 259-275.

[16] Romero-Gómez P, Choi CY, Lopez-Cruz IL. Enhancement of the greenhouse air ventilation rate under climate conditions of central Mexico. Agrociencia. 2010; 44: 1-15.

[17] Boulard T, Kittas C, Roy JC, Wang S. Convective and ventilation transfers in greenhouses, part 2: Determination of the distributed greenhouse climate. Biosystems Engineering. 2002; 83: 129-147.

[18] ANSYS-FLUENT. Fluent user guide. Lebanon: Ansys Inc., 1998.

[19] ASHRAE. Applications handbook: American Society of Heating, Refrigeration and Air Conditioning Engineers. Atlanta: ASHRAE, 1999.

[20] Willits DH, Li S, Yunker CA. The cooling performance of natural ventilated greenhouse in the southeastern US. Proceedings of the International Symposium on Greenhouse Cooling. Acta Horticulturae 2006; 719: 73-80.

[21] Montero JI, Hunt GR, Kamarudddin R, Anton A, Bailey BJ. Effect of ventilator configuration on wind driven ventilation in a crop protection structure for the tropics. Agricultural Engineering Research. 2001; 80: 99-107.

[22] Kittas C, Karamanci M, Katsoulas N. Air temperature in a forced ventilation greenhouse with rose crop. Energy and Buildings. 2005; 37: 807-812. 
[23] Kittas C, Katsoulas N, Baille A. Influence of greenhouse ventilation regime on microclimate and energy partitioning of a rose canopy during summer conditions. Agricultural Engineering Research. 2001; 79: 349-360.

[24] Baeza EJ, Pérez-Parra JJ, Montero JI. Effect of ventilator size in natural ventilation in parral greenhouse by means of CFD simulations. Acta Horticulturae. 2005; 691: 465472.

[25] Kacira M, Sase S, Okushima L. Effects of side vents and span numbers on wind-induced natural ventilation of a gothic multi-span greenhouse. Japan Agricultural Research Quarterly. 2004; 38: 227-233.

[26] Bartzanas T, Kittas C, Boulard T. Effect of vent arrangement on windward ventilation of a tunnel greenhouse. Biosystems Engineering. 2004; 88: 479-490.

[27] Ould K, Bournet PE, Migeon C, Boulard T, Chasseriaux G. Analysis of greenhouse ventilation efficiency based on computational fluid dynamics. Biosystems Engineering. 2006; 95: 83-98.

[28] Baeza EJ. Optimization of ventilation systems design for greenhouse parral-type. $\mathrm{PhD}$ thesis, University of Almeria, 2007.

[29] Teitel M, Tanny J. Radiative heat transfer from heating tubes in a greenhouse. Agricultural Engineering Research. 1998; 69: 185-188.

[30] Popovski K. Location of heating installations in greenhouses for low temperature heating fluids. In: Industrial thermal effluents for greenhouse heating, European cooperative networks on rural energy, 1st ed., Rome, 1986, 51-53.

[31] Roy JC, Boulard T, Bailley Y. Characterization of the heat transfer from heating tubes in a greenhouse. In: E-Proceedings Symposium AGENG, Warwick, 2000.

[32] Kempkes F, Van de Braak NJ. Heating system position and vertical microclimate distribution in a chrysanthemum greenhouse. Agricultural and Forest Meteorology. 2000; 104: 133-142.

[33] Piscia D, Montero JI, Baeza E, Bailey BJ. A CFD greenhouse night-time condensation model. Biosystems Engineering. 2012; 111: 41-154.

[34] Kim K, Yoon JY, Kwon HJ, Han JE, Son JE, Nam SW, Giacomelli GA, Lee IB. 3-D CFD analysis of relative humidity distribution in greenhouse with a fog cooling system and refrigerative dehumidifiers. Biosystems Engineering. 2008; 100: 245-255.

[35] Boulard T, Haxaire R, Lamrani MA, Roy JC, Jaffrin A. Characterization and modelling or de air fluxes induced by natural ventilation in a greenhouse. Agricultural Engineering Research. 1999; 74: 135-144.

[36] Tadj N, Draoui B, Theodoridis G, Bartzanas T, Kittas C. Convective heat transfer in a heated greenhouse tunnel. Acta Horticulturae. 2007; 747: 113-120. 
[37] Alves I, Perrier A, Pereira LS. Aerodynamic cover and surface resistances of complete cover crops: how good is the "big leaf"? Transactions of the ASAE. 1998; 41: 345-351.

[38] Miguel AF, Vande-Braak NJ, Bot GP. Analysis of the airflow characteristic of greenhouse screening materials. Research in Agricultural Engineering. 1997; 67: 105-112.

[39] Hsu C, Cheng P. Thermal dispersion in a porous medium. International Journal of Heat and Mass Transference. 1990; 33: 1587-1597.

[40] Haxaire R. Characterization and modelling of the air flows within a greenhouse. PhD thesis, University of Nice Sophia Antipolis, 1999.

[41] Haxaire R, Boulard T, Mermier M. Greenhouse natural ventilation by wind forces. Acta Horticulturae. 2000; 534: 31-40.

[42] Lee IB, Short TH. Two-dimensional numerical simulation of natural ventilation in a multi-span greenhouse. Transaction of the ASAE. 2000; 43: 745-753.

[43] Fatnassi H, Boulard T, Bouirden L. Simulation of climatic conditions in full-scale greenhouse fitted with insect-proof screens. Agricultural and Forest Meteorology. 2003; 118: 97-111.

[44] Molina-Aiz FD, Valera DL, Alvarez AJ. Measurement and simulation of climate inside Almeria-type greenhouse using computational fluid dynamics. Agricultural and Forest Meteorology. 2004; 125: 33-51.

[45] Wilson JD. Numerical Studies of flow through a windbreak. Journal of Wind Engineering \& Industrial Aerodynamics. 1985; 21: 119-154.

[46] Molina-Aiz FD, Valera DL, Alvarez AJ, Madueño A. A wind tunnel study of airflow through horticultural crops: determination of the drag coefficient. Biosystems Engineering. 2006; 93: 447-457.

[47] ANSYS-FLUENT. Fluent user guide. Lebanon: Ansys Inc., 1997.

[48] Flores-Velazquez J, Montero JI. Computational fluid dynamics CFD study of largescale screenhouse. Acta Hortculturae. 2008; 797: 117-122.

[49] Stanghellini C. Transpiration of greenhouse crops: an aid to climate management. PhD thesis, Agricultural University of Wageningen, 1987. 
Chapter 5

\title{
Technologic Appliance and Performance Concerns in Wheelchair Racing - Helping Paralympic Athletes to Excel
}

\author{
Pedro Forte, Tiago M. Barbosa and Daniel A. Marinho \\ Additional information is available at the end of the chapter
}

http://dx.doi.org/10.5772/61806

\begin{abstract}
Numerical simulations have provided useful evidence in helping several sportsmen to excel in their field. This methodology aims to have a deeper understanding on the influence of equipment and sports techniques on sports performance. In wheelchair racing, technology was used without considering specific sport (some of the Paralympic sports used the same technology of their Olympic counterparts). It has induced unique changes in prosthetic and wheelchair devices. Eventually, technology has become an essential part of Paralympic sports, wheelchair-racing being one of the most popular events. Numerical simulations can help us gather evidence on the effects of drag force acting upon the athlete-chair system.

Different types of wheelchairs are designed for racing (track and road races), net, and invasion sports. One of the various strategies to enhance performance is to minimize the aerodynamic drag of the frame, tires, helmet, sports outfit, and body posture.

Numerical simulations can be used to predict the fluid dynamics.

The goal of this chapter is to review the state-of-the-art numerical simulations and suggest further studies in wheelchair racing. The chapter will include sections covering: (i) main determinants in wheelchair racing; (ii) the effect of aerodynamic force in in wheelchair racing performance; (iii) analytical models, experimental testing, and numerical simulations in wheelchair racing; and (iv) numerical simulations on equipment and techniques.
\end{abstract}

Keywords: CFD, Paralympic, Performance 


\section{Introduction}

Wheelchair racing is a major event in Paralympics. This sport holds short $(100 \mathrm{~m})$ to long distance $(42 \mathrm{~km})$ races. The athletes are classified according to their condition into classes (T5i, i.e., $\mathrm{i}=1,2,3$ and 4 with injuries at C5-6, C7-8, T1-7 and T8-S4 respectively) [1, 2]. In wheelchair racing, sports science has been expressed as a need in the modeling and computer simulations of racing wheelchairs and the racing itself $[3,4]$.

The first game for disabled persons, in pair with the Olympic Games, was held in Rome in 1960. Pioneer research on wheelchair racing happened in the 1980s which was based on highspeed films at laboratory or running track settings (5). In the 1980s, manufacturers estimated that more than 10,000 racing wheelchairs commercialized worldwide [6]. Being increasingly popular, the competitions between the contenders in these sports became tighter and research is eagerly needed to help Paralympic athletes to excel.

Nowadays, most world-ranked Paralympic athletes, including wheelchair racers, develop an evidence-based practice with the help of coaches and sports analysts. Every detail of a race is deeply examined to have a deeper insight of the determinant factors that might help them excel. The resistance acting on the wheelchair racer is one of the major concerns for practitioners. The resistance force having an opposite direction to the displacement must be minimized so that for a given amount of thrust, the subject can reach a higher acceleration and speed. Lately, a few research projects were reported in the literature under this topic. So, the aim of this chapter is to review the state-of-the-art numerical simulations in wheelchair racing and suggest further studies in this sport.

\section{The wheelchair race}

The stroke cycle in wheelchair racing is divided into two phases: the propulsion and the recovery phases (Figure 1). The propulsion phase is characterized by the tangential force applied to the handrim. In the beginning of the race, this phase should be its highest and longest contact time so that the mechanical impulse will also be high [7]. It represents $33 \%$ to $35 \%$ of the full stroke cycle. The remaining $65 \%$ to $67 \%$ of the stroke time is the recovery phase in elite wheelchair racers $[8,9,7,10]$. In this phase, we have the time period that the hand does not contact the rims. The hand tends to follow different paths until being positioned in the rim again to a new propulsion phase [11-13]. However the recovering phase may change, the free chosen push frequency (the preferred stroke frequency adopted by each subject) ranged from 32 to 86 pushes $/ \mathrm{min}$ at a $6.58 \mathrm{~m} / \mathrm{s}$ in wheelchair racing.

The stroke cycle can also be divided into 5 moments: catch, drive, release, lift and stretch, and finally, acceleration. Catch is the hand contact moment on the handrim, it usually occurs nearly the 1 and 2 o'clock positions. Drive phase is the moment of the hand and wrist acceleration on the handrim and it usually occurs nearly the 2 and 5 o'clock. Release phase is the moment of the contact breaking of the hand with the handrim, closer to the $6 o^{\prime}$ clock. Lift and stretch phase 
is characterized by the elbow flexion and elevation in the sagittal plan. Acceleration moment is when the elbow extension is done before the handrim contact.

\section{Wheelchair Racing Stroke Phases}

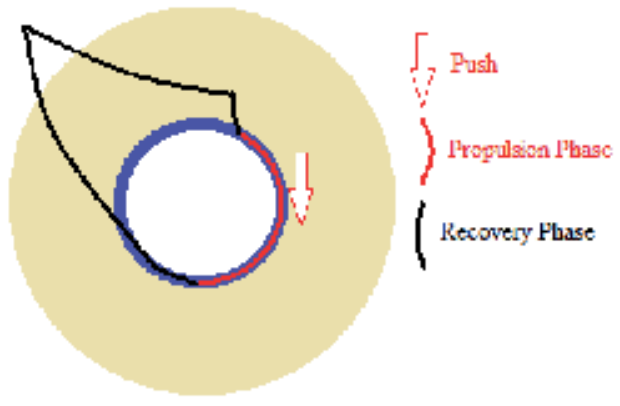

Figure 1. Stroke Cycle in Wheelchair Racing

During a race, the racers aim to reach the maximal speed as soon as possible and keep it for the remaining time (Figure 2). Figure 2 also depicts the intra-cycle speed (i.e. due to hand contact and recovery phase). This only proves that propulsive forces are higher than resistance forces (i.e. positive acceleration) at a given moment, and there are also moments that it will have higher intensity (i.e. negative acceleration).

\section{Speed Increase in a 10sg Race and intra-stroke speed mean}

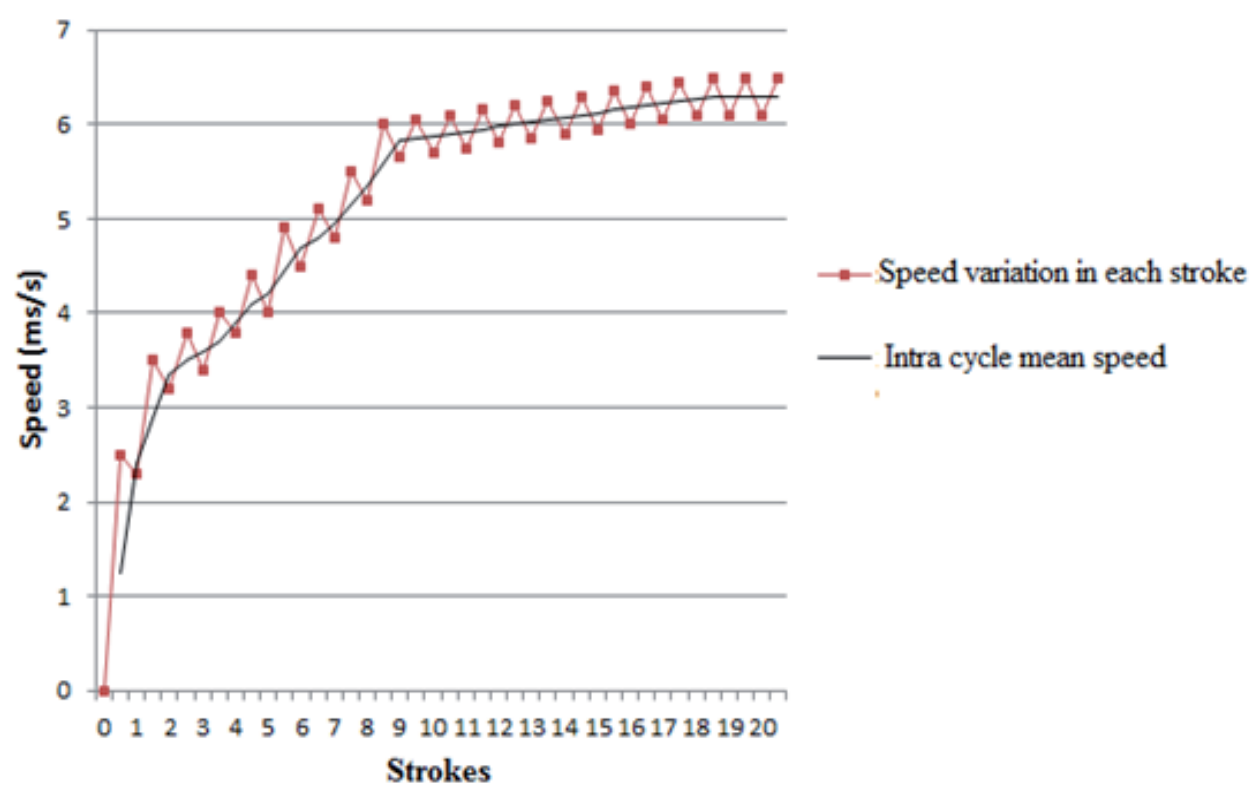

Figure 2. Theoretical Representation of the Speed over Time in 20 Stroke Cycles 
Stroke kinematics is selected on a regular basis to report the stroke cycle. This includes the assessment of the average speed, stroke frequency, and stroke length.

The stroke frequency can be evaluated by measuring the number of cycles per minute and stroke speed given by the product of stroke length and stroke frequency. Stroke length is the difference between the contact distance of a stroke and the contact covered distance of the next stroke. Thus, stroke length can be explained as the covered distance with handrim contact in one stroke [14]. The average speed is calculated dividing the stroke length by the stroke frequency [14].

A higher stroke frequency combined with the hand linear velocity will lead to a greater energy cost, and probably will enhance cardiorespiratory stress affecting blood lactate and heart rate [15]. The increase of speed is related with blood lactate concentration and rise of heart rate. Despite this, the increase of the handrim diameter seems to reduce the blood lactate concentration at $20 \mathrm{~km} / \mathrm{h}$, however it did not happen at $22 \mathrm{~km} / \mathrm{h}$. [15].

An increased time of hand contact on the handrim without wrist acceleration will generate deceleration by friction resistance applied by the hand on the handrim. Therefore, to achieve a lower contact, it is recommended to produce a high speed soon after the start [16], which is possible to improve as demonstrated by sprinters in strength development [17]. The elbow motion seems to range from $60.9^{\circ}$ to $5.2^{\circ}$ in flexion to extension movement; and the maximum flexion velocity ranges from $515.4 \% / \mathrm{s}$ and $572.8 \%$, which is independent of the lightweight of the wheelchair. However, the propulsion arc reduces by $12^{\mathrm{o}}$ to $14^{\mathrm{o}}$ in the pumping-stroke technique that results from the shortest handrim contact possible, usually at the start of the race [18]. In the 1986 National Wheelchair Track and Field Championship in Illinois, in elite finalists both in paraplegic and quadraplegic wheelchair racers, the mean velocity-time ranged in each stroke from $5.1 \mathrm{~m} / \mathrm{s}$ to $5.5 \mathrm{~m} / \mathrm{s}$ and $3.7 \mathrm{~m} / \mathrm{s}$ to $4.27 \mathrm{~m} / \mathrm{s}$ respectively. The mean velocity in each stroke ranged $0.4 \mathrm{~m} / \mathrm{s}$ and $0.6 \mathrm{~m} / \mathrm{s}$ between the propulsion and recovery phase for the same subjects respectively. As the stroke frequency increases, velocity peak also enhances, despite the negative correlation between the velocity peak and the hand contact time with the handrim. The contact phase of the hand in handrim ranged from $13.58^{\circ}$ to $15.6^{\circ}$ for quadriplegics and paraplegics. The start acceleration phase at the rims starts at $48.54^{\circ}$ and $71.1^{\circ}$. The break contact happens at $187.5^{\circ}$ and $238.9^{\circ}$. The peak velocity for each stroke, occurs at $181.1^{\circ}$ and $223.5^{\circ}$ for quadriplegics and paraplegics respectively. These results were founded in a 10-second sprint with 20 to 25 propulsive cycles (strokes) for each subject [19].

The velocity peak seems to occur near the 10-second sprint in wheelchair track racers [7]. Wheelchair basketball players achieve $80 \%$ of the peak velocity in the first three strokes. On the other hand, wheelchair racers at the third stroke only achieve $55 \%$ of the peak velocity. The stroke frequency in short distances is greater than in long distances. In 10 seconds, sprints were obtained with 2 strokes per second [20]. However, in an 800m race, the stroke frequency ranged between 1.77 and $1.72 \mathrm{~Hz}$ [7]. 


\section{Main determinants in wheelchair racing}

The wheelchair racing athlete intends to reach the maximum acceleration as soon as possible. According to Newton's Second Law, the acceleration (a) is the ratio between force ( $\mathrm{f}$ ) and mass (m) (Equation 1).

$$
a=\frac{F}{m}
$$

In the wheelchair racing case, it is also possible to say that:

$$
a=\frac{\left(F_{\text {Prop }}-F_{\text {Resist }}\right)}{m}
$$

$\mathrm{F}_{\text {Prop }}$ and $\mathrm{F}_{\text {Resist }}$ are the propulsive forces and the resistive ones respectively.

Positive acceleration is obtained by the applied propulsive forces on the wheels, overcoming the resistive ones (Figure 3). In wheelchair racing, the propulsive force is the push on the handrim, generating motion (i.e. the applied force by the ground in the wheel). The resistive forces are the rolling friction $(\mathrm{Fr})$ and aerodynamic drag $(\mathrm{Fd})$.
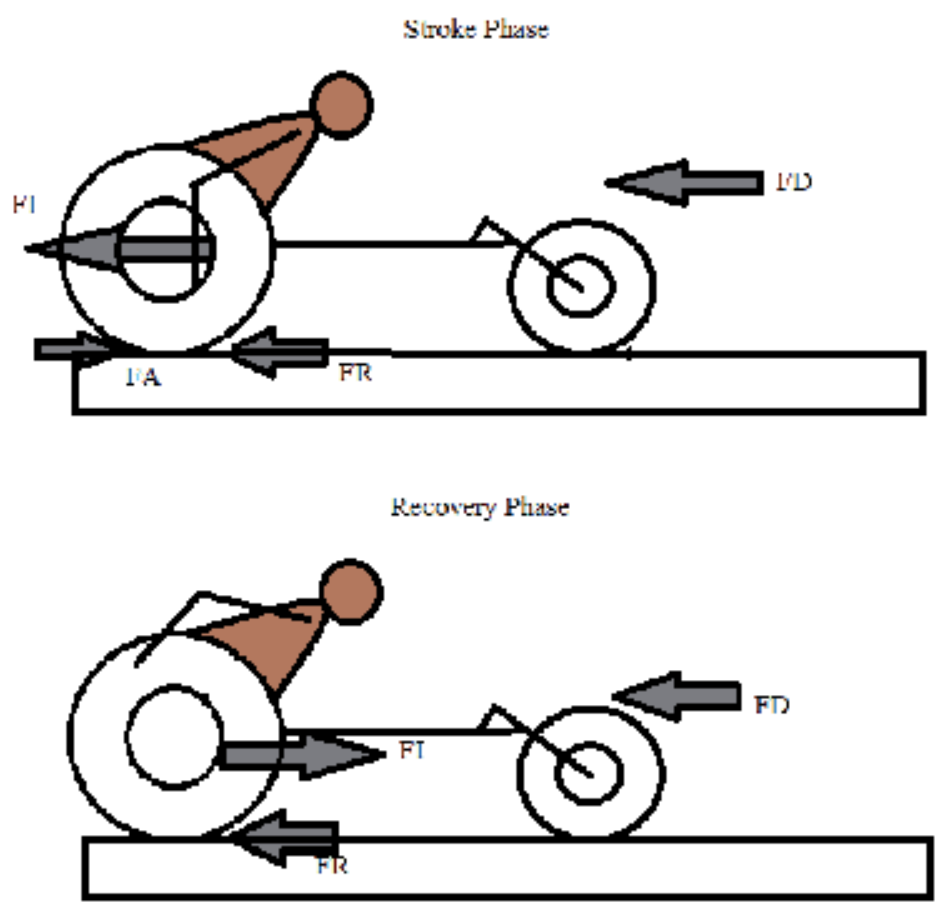

Figure 3. Free body diagram in wheelchair racing [21]; FI - Inertial force; FD - Drag force; FR - Rolling friction forces; FA - The applied force by the ground on the rear wheels. 
Fuss [21] reported an analytical model to describe the performance based on these major determinants. The inertial force is the required force to change the body state and start the motion. The drag force is the air resistance to the wheelchair-athlete system. Rolling friction is the resistive force of the ground on the wheels' tires. Considering Newton's Third Law, the applied force by the ground on the rear wheel is the opposite one of the wheel in the ground derived by propulsion.

According to Fuss [21], the total energy expended by the athlete is taken by rolling friction and air drag. It is possible to say that the velocity is dependent on the kinetic energy of the system and its mass.

$$
v=\sqrt{\frac{2\left(E_{\text {in }}-E_{\text {loss }}\right)}{m}}
$$

In the equation, $\mathrm{v}$ is the velocity, $\mathrm{E}_{\mathrm{in}}$ is the energy produced by the athlete and $\mathrm{E}_{\text {loss }}$ is the energy lost.

$$
\mathrm{E}_{\text {kin }}=\mathrm{E}_{\text {input }}-\left(\mathrm{E}_{\text {drag }}+\mathrm{E}_{\text {friction }}\right)
$$

The total kinetic energy is obtained from the sum of all mobile parts with a speed higher than zero. It is also considered that other movement oscillations do not contribute to the kinetic energy. Then:

$$
E_{k i n}=\frac{m v^{2}+\sum_{i=1}^{3} I_{i} \omega_{i}}{2}
$$

In Equation 5, Ii is the moment of inertia and $\omega \mathrm{i}$ is the angular velocity of wheel $\mathrm{i}$.

When the inertia force (FI) gets combined with distance, the kinetic energy (Ekin) can be formulated as:

$$
E_{k i n}=\int_{x 1}^{x 2} F_{I} d_{x}
$$

Equations 7 and 8 consider that velocity comes from the work of the rear wheel ( $\omega r i)$ and acceleration is the angular velocity of the rear wheel $(\mathrm{a}=\alpha \mathrm{ri})$, then solving it for FI, we obtain:

$$
F_{I}=a\left(m+\sum_{i=1}^{3} \frac{I_{i}}{r_{i}^{2}}\right)
$$


In this formula, $\mathrm{m}$ is the mass of wheelchair-athlete system and I is obtained from the relation between the one wheel mass and the wheel radius gyration squared. The mass of one wheel multiplied by its radius of gyration squared when accelerated, an equivalent mass results in FI:

$$
M=m+\sum_{i=1}^{3} \frac{I_{i}}{r_{i}^{2}}
$$

The propulsion cycle is given by the stroke and recovery phases, the equilibrium forces in the stroke one are given by:

$$
\begin{gathered}
F_{A}+F_{I}+F_{D}+F_{R}=0 \\
F_{A}+M_{a}+C_{D v^{2}}+m g \mu_{R}+m g k_{f v^{2}}=0
\end{gathered}
$$

$C_{D}$ is the drag coefficient, $v$ is the instantaneous velocity (considering distance and time at a given moment) and the acceleration $\left(\mathrm{d}^{2} \mathrm{x} / \mathrm{dt}^{2}\right)$. The $\mathrm{C}_{\mathrm{D}}$ cluster is obtained from 0.50cDA, being the product of air density (Q) with the drag coefficient of the air $\left(C_{D}\right)$ and the frontal surface area (A). Considering that wheelchairs are in straight line and according to Equation 14, 12 and 13, FR is given by:

$$
F_{R}=\mu_{R} m g+k_{f} m g v^{2}
$$

Where $\mu_{\mathrm{R}}$ is the rolling friction coefficient, $\mathrm{m}$ is the mass, $\mathrm{g}$ the gravitational acceleration and $\mathrm{k}_{\mathrm{f}}$ the coefficient of speed.

$$
F_{A}+M_{a}+c_{1} v^{2}+c_{2}=0
$$

$\mathrm{C}_{1}$ is given by,

$$
c_{1}=C_{D}+m g k_{f}
$$

And C2,

$$
c_{2}=m g \mu_{R}
$$

Thereby, in a wheelchair-athlete system, the responsible variables for the energy losses are FD and FR and both forces are equal to zero in a loss-free environment. 


\section{Propulsion}

In the propulsion phase, Fuss [21] described four forces as the main ones. The ground applied one at the rear wheel, aerodynamic drag force and the rolling friction one. When the mass gets accelerated, it produces an inertial force (FI). The kinetic energy derived from FI, is stored on the wheels, it depends of the moment of inertia and the angular speed of the wheels creating the system motion. The four forces in equilibrium are described as,

$$
F_{A}+F_{I}+F_{D}+F_{R}=0
$$

$\mathrm{F}_{\mathrm{A}}$ is the force applied to the ground on the wheels derived from the tangential force applied on the handrim $\left(\mathrm{F}_{\mathrm{I}}\right)$. For movement occurring, $\mathrm{F}_{\mathrm{A}}$ must be higher than $\mathrm{F}_{\mathrm{J}}, \mathrm{F}_{\mathrm{D}}$ and $\mathrm{F}_{\mathrm{R}}$.

Several investigations have been made to better understand the physiological factors and demands that may affect the wheelchair propulsion. Others, for instance, [10] presented the motion of the racing wheelchair derived by the sum of all external forces by:

$$
\mathrm{F}\left(\mathrm{R} / \mathrm{r}_{\mathrm{pr}}\right)=\mathrm{My}+\mathrm{Iv} / \mathrm{R}+\mathrm{I}_{\mathrm{f}} \mathrm{v} / \mathrm{r}+\mathrm{Fa}+\mathrm{M}_{\mathrm{B}} / \mathrm{r}_{\mathrm{ax}}+\mathrm{M}_{\mathrm{b}} / \mathrm{r}_{\mathrm{axf}}+\mathrm{F}_{\mathrm{R}}+\mathrm{F}_{\mathrm{r}}+\mathrm{W} \sin 0(\mathrm{x})
$$

Where, " $\mathrm{R} / \mathrm{r}_{\mathrm{pr}}$ " represents the gear ratio of the handring to the wheel. $\mathrm{F}$ - The tangential force in the handrings; $R$ and $r_{p r}$ - The radius of the rear wheels and handrings; $M$ - The mass of the system athlete-wheelchair; $\mathrm{v}$ - The velocity of the athlete-wheelchair system, given by: $\mathrm{v}=\mathrm{x}=$ $\mathrm{dx} / \mathrm{dt}$; I and $\mathrm{I}_{\mathrm{f}}$ - The inertia of the rear wheels and the wheels inertia; $\mathrm{r}$ - The radius of the front wheels; $\mathrm{Fa}$ - The air resistance of the athlete-wheelchair system; $\mathrm{M}_{\mathrm{B}}$ - The rear hubs bearing resistance; $M_{b}$ - The front hubs bearing resistance; $r_{a x}$ and $r_{a x f}$ - The radius of the rear and front axles respectively; $F_{R}$ and Fr- The rolling resistance of the rear and front wheels respectively; $\mathrm{W}$ - The weight of the athlete-wheelchair system; $\Theta(\mathrm{x})$ - The inclination angle (i.e., changes in elevation); $\mathrm{x}$ - The covered distance;

\section{Inertia}

The sum of the kinetic energy to all mobile parts is given by Equation 5. It includes the energy of the three wheels and the translational energy. The kinetic energy is equal to the inertial force combined with distance (Equation 6). Thus, the inertial force $\left(\mathrm{F}_{\mathrm{I}}\right)$ is given by:

$$
F_{I}=a M
$$

M represents the sum of the inertial moments.

The Inertial Force is the required force for a body state change. At the beginning of the wheelchair race, the inertial force is the required force for starting the wheelchair. Therefore, it is clear that the mass influences the inertial moment in the system and that the mass reduction 
will lead to a sooner and/or higher speed. Fuss [21] reported that a reduction of $1 \mathrm{~kg}$ in a wheelchair-athlete system will improve the winning time by 0.132 sg.

\section{Air drag and rolling resistance}

In wheelchair racing, the air drag results from the air resistance in the surface area of the athletewheelchair system. In contrast, the rolling resistance results from the friction of the tyres on the ground. Aerodynamic resistance can be minimized by reducing the frontal area of the subject, intending to improve the winning time of a racing wheelchair. At speeds greater than $5 \mathrm{~m} / \mathrm{s}$ aerodynamic drag represents $90 \%$ of the resistive forces [22]. Between $5.32 \mathrm{~m} / \mathrm{s}$ and $6.83 \mathrm{~m} /$ $\mathrm{s}$, rolling resistance is greater than aerodynamic drag with a partial contribution of $65 \%$ to $75 \%$ and $25 \%$ to $35 \%$ respectively. At greater speeds, aerodynamic drag starts to represent a greater contribution of the resistive forces [23].

Air drag and rolling resistance can be acceded from Equation 18 for rolling resistance and Equation 19 for air drag:

$$
\begin{gathered}
F_{R}=\mu_{g} m g+k_{f} m g v^{2} \\
F_{D}=0.5 \rho A_{d} v^{2} C_{D}
\end{gathered}
$$

In these two equations, $F_{R}$ is the rolling friction, $\mu_{\mathrm{g}}$ is the rolling friction coefficient, $\mathrm{m}$ is the mass of the athlete-wheelchair system, $g$ is the gravity acceleration and $k_{f}$ is the coefficient of the speed influence in rolling resistance force, $\mathrm{Q}$ is the air density, $\mathrm{A}_{\mathrm{d}}$ is the frontal area, $\mathrm{v}^{2}$ is the velocity, and $C_{D}$ is the drag coefficient.

Lightweight clothes should be worn by wheelchair racers to reduce the aerodynamic drag [23]. A flexed upper trunk position also reduces the aerodynamic drag, as does reducing the rear wheel spokes to 24 [22]. In cycling, a flexed upper trunk position reduces the frontal area in $20 \%$ to $29 \%$ [24]. In wheelchair racing, the same position reduces the frontal area in $3 \%$ to $4 \%$ [25].

Mass would also influence the rolling friction; reducing the wheelchair-athlete system would also improve the speed, mainly in short distances. A $1 \mathrm{~kg}$ reduction would improve $1-2.3 \%$ of the winning time in wheelchair racing [21].

\section{Analytical models, experimental testing, and numerical simulations in wheelchair racing}

\subsection{Experimental testing: Coast-down technique}

Aerodynamic and rolling resistance can be tested providing realistic opportunities that cannot be achieved in laboratory. The coast-down methods founded in literature are: (i) roll distance 
applying the ramp methods; (ii) the timing gate method that measures the velocity decrease in different points between two marks (gates); and (iii) the velocity method that measures directly the speed in each time.

The roll distance applying the ramp method consists rolling, a wheelchair from a ramp to the field, measuring the covered distance by the wheelchair [26]. The timing gate method was developed as an alternative to the roll distance, produced in less time and space compared with the whole roll until the wheelchair stops. Two marks should be performed counting the time when the wheelchair passes between them [27]. In the velocity method, the racer reaches the maximum speed, stops the propulsion and the velocity is recorded in each instance [27, 21]. A dynamometer-based coast-down test was used for the wheel deceleration calculus [28]. For wheel deceleration in each trial, hub (Hubxyz) and handrim (Rimxyz) markers were placed. The wheel rotation on each time was assessed by the law of cosines:

$$
\theta=\cos ^{-1}\left(\frac{A \times B}{\|A\|\|B\|}\right)
$$

A = Rimxyz(i) - Hubxyz(i); B = Rimxyz (i + 1) - Hubxyz (i + 1).

To avoid wheel size differences, the angular speed was converted to linear one. It has fitted a line to the linear velocity $(\mathrm{v})$ data intending to determine the wheel deceleration (ad), the time to coast down from $2 \mathrm{~m} / \mathrm{s}$ to $1 \mathrm{~m} / \mathrm{s}$ is represented by " $\mathrm{t}$ " and the initial wheel velocity is represented by " $\mathrm{v}_{0^{\prime \prime}}$.

$$
v=a_{d} t+v_{0}
$$

The estimation for rolling resistance can be done based on Cooper's method [10]. Intending to simplify the method, aerodynamic drag, wheel and roller bearing resistances, as other external resistances were neglected. Thus, the rear wheel motion was described by:

$$
\mathrm{T}_{\mathrm{w}}=\left(\mathrm{I}_{\mathrm{r}} \frac{\mathrm{R}}{\mathrm{r}} \mathrm{I}_{\mathrm{w}}\right) \mathrm{a}_{\mathrm{w}}+\mathrm{F}_{\mathrm{RR}} \mathrm{R}
$$

Where, " $\mathrm{T}_{\mathrm{w}}$ " was the torque applied by the hand to the rear wheel; " $\mathrm{I}_{\mathrm{r}}$ " and " $\mathrm{I}_{\mathrm{w}}$ " were the moment of inertia of the roller and the rear wheel, respectively; " $r$ " and " $R$ ", the roller and rear wheel radius, respectively; " $\mathrm{a}_{\mathrm{w}}$ " and " $\mathrm{F}_{\mathrm{RR}}$ " as the angular acceleration of the rear wheel and the rolling resistance force respectively.

Considering that the propulsive force was discontinued, " $\mathrm{T}_{\mathrm{w}}$ " is equal to zero, and " $\mathrm{a}_{\mathrm{w}}$ " represents the wheel angular deceleration. The equation is being used to describe the rear wheel rotation. The roller inertial moments $\left(0.87 \pm 0.15 \mathrm{~kg}-\mathrm{m}^{2}\right)$ and wheels $\left(0.12 \pm 0.02 \mathrm{~kg}-\mathrm{m}^{2}\right)$ were experimentally acceded resourcing the acceleration method by DiGiovine et al. [29]. 
Applying the " $\mathrm{I}_{\mathrm{r}}$ " and " $\mathrm{r}$ " values into Equation 22, and rewriting " $\mathrm{a}_{\mathrm{w}}$ " as the ratio between linear deceleration and radius $\left(a_{d} / R\right)$, rolling resistance force is obtained from the wheel radius, inertia, and deceleration, calculated by:

$$
\mathrm{F}_{\mathrm{RR}}=\frac{-\mathrm{a}_{\mathrm{d}}}{\mathrm{R}^{2}}\left(\mathrm{I}_{\mathrm{w}}+5.47 \mathrm{R}\right)
$$

Coast-down distance (CDD) calculation was computed using the equation of motion, where acceleration is assumed to be constant and $t$ is the time for coast-down technique ends, when the wheelchair stops:

$$
\mathrm{CDD}=\frac{1}{2} \mathrm{a}_{\mathrm{d}} \mathrm{t}^{2}+\mathrm{v}_{0} \mathrm{t}
$$

Another method exists in accelerating the wheelchair (e.g., from 2.5 to $12.8 \mathrm{~m} / \mathrm{s}$ ). The rider stops the propulsive phases reaching the target speed counting the length and/or time until the wheelchair stops [30]. In this method both aerodynamic drag and rolling resistance are obtained. Figure 4 depicts a speed decay of wheelchair considering the velocity method, measuring the speed in each time.

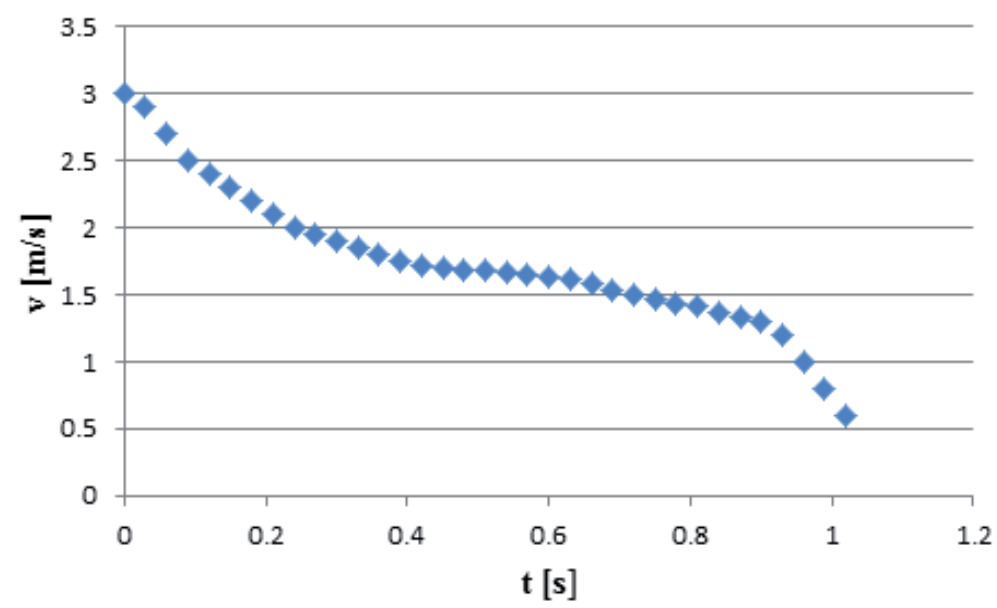

Figure 4. The Velocity Decay of a Manual Wheelchair over a Typical Trial

\subsection{Wind tunnel testing}

At least one paper can be found in the literature reporting the assessment of the aerodynamics in wind tunnel between two wheelchair models [31]. The drag forces were measured for speeds between $54.86 \mathrm{~km} / \mathrm{h}$ and $164.60 \mathrm{~km} / \mathrm{h}$ with intervals of $18.28 \mathrm{~km} / \mathrm{h}$. However, air viscosity and 
ground motion effect are not taken into account with this methodology. Aside from this, a better wind tunnel test should be performed intending to determine the best helmet and clothes to use in races [31]. The Drag was proportional with the speed in the two models A and B. Model A presented a strict nose, with fenders at the wheels and an exceeded front angle attack. The drag force was acceded in grams (gr) and ranged from 26(gr) to 360(gr) in both models, with and without pilot. With and without pilot, Model B presented a lower drag force for velocities lower than $91.44 \mathrm{~km} / \mathrm{h}$. At higher speeds, Model A presented lower drag force with and without pilot.

From this experimental test, it is possible to confirm that specific wheelchairs should be made for specific racers, and a sprint race wheelchair should be different to a long race wheelchair.

\subsection{Analytical method for drag assessment}

For rolling friction and aerodynamic drag, Burton, Fuss \& Subic [32] presented an analytical procedure to estimate rolling friction and aerodynamic drag. FR is non-linear when calculated from visco-elastic models, considering the deformation of the tyres, reduces in the ground higher speed in parabolic function. The equations for these two forces are expressed in Equations 18 and 19.

According to Fuss [21], based on vehicles' data [33], consider that the $\mu_{\mathrm{g}}$ of a racing wheelchair is $0.01, \mathrm{~K}_{\mathrm{f}}$ is $5 \times 10^{-6} \mathrm{~s}^{2} \mathrm{~m}^{-2}$, a mean speed of $10 \mathrm{~m} / \mathrm{s}$ and a mass athlete-wheelchair system of $80 \mathrm{~kg}$ the first and second term of the equation 22 is 7.85 and $0.35 \mathrm{~N}$.

In a partial contribution assessment of air drag, Barbosa et al. [23] assumed the air density of $1.2041 \mathrm{~kg} / \mathrm{m} 3$ in the sea level at $20^{\circ} \mathrm{C}$. The surface area was measured with the photogrammetric technique in the frontal plane and the drag coefficient was assumed to be 0.7 . All estimations were completed in each speed moment between $0 \mathrm{~m} / \mathrm{s}$ and $13 \mathrm{~m} / \mathrm{s}$ increasing in every $0.1 \mathrm{~m} / \mathrm{s}$. considering the world speed record, the air drag represents $34.89 \%$.

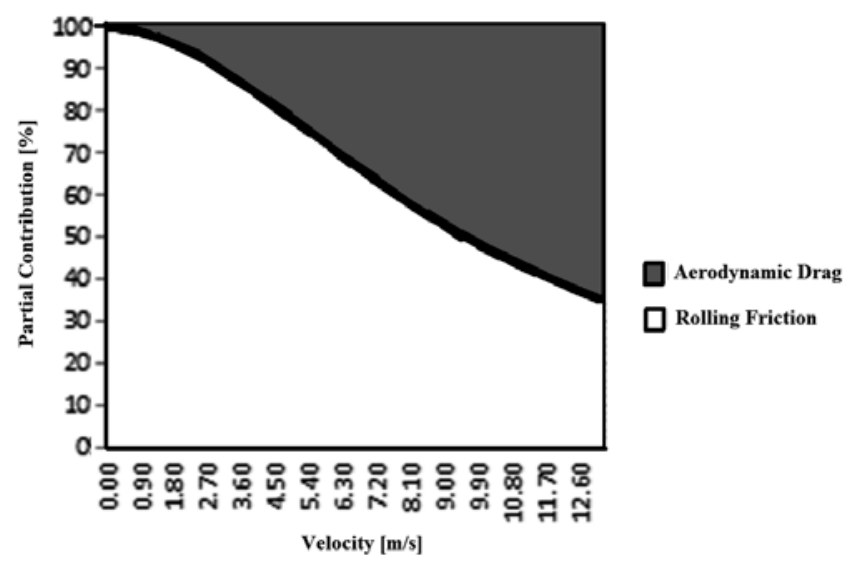

Figure 5. Partial Contribution of Aerodynamic Drag and Rolling Friction by Barbosa et al. [23]. 


\subsection{Numerical simulations: Computer fluid dynamics}

Computational Fluid Dynamics (CFD) has been used over the last 20 years. There are a lot of benefits on CFD and tools, such as FLUENT, CFX, STAR-CD and FiDAP, are all commercially available and used in industrial settings in the engineering community since the 1990s. Engineers and scientists started to use these simulations in competitive sports to reach a performance advantage, to improve sports equipment design and elite athletes' aerodynamics or hydrodynamic enhancements [34].

In sport sciences, the CFD presented concordance between numerical simulations and in vivo tests. For the simulation, a 3D body model scan is required and the images processing can be made with recourse to Anatomics Pro (Anatomics, Kannapolis, NC, USA) and FreeForm (Sensable Technologies, Woburn, MA, USA). The scan files are saved as IGES (*.igs) format, intending to be executable in Gambit/FLUENT (FLUENT Inc, Hanover, NH, USA). In Gambit/ FLUENT, it is possible to generate the greed and to define the finite elements in $3 \mathrm{D}$ areas.

The numerical simulation consists discretization of Navier-Stokes equations by the finites volumes methods. These equations come from Newton's Second Law in fluid mechanics, assuming that the fluid stress is the sum of diffusion of its viscosity, resulting from an applied pressure term. The equation resolution determines the fluid speed in a determined point at space and time. CFD is based on an approximated finite volume. In this approximation, space is divided into small cells to form a mesh or greed, applying a solver algorithm for the equations of fluid volume motion resolution $[35,36]$.

The Reynolds-Averaged Navier-Stokes (RANS) comes from decomposing the instantaneous values into means and/or fluctuating components. Fluid flow behavior (Equation 25), Reynolds stresses (Equation 26), temperature (Equation 27) and mass transfer (Equation 28) can be solved in this methodology.

$$
\begin{gathered}
\frac{\partial_{\mathrm{Ui}}}{\partial_{\mathrm{xi}}}=0 \\
\frac{\partial_{U i}}{\partial_{t}} \pm U_{j} \frac{\partial_{U i}}{\partial_{x_{j}}}=-\frac{1}{\rho} \frac{\partial P}{\partial x_{j}}+\frac{\partial}{\partial x_{j}}\left(2 v S_{i j}-\overline{\mu_{j}^{\prime} \mu_{i}^{\prime}}\right) \\
\frac{\partial_{\theta i}}{\partial_{t}} \pm U_{j} \frac{\partial_{\theta}}{\partial_{x_{j}}}=\frac{1}{\rho_{c p}} \frac{\partial}{\partial x_{j}}\left(k \frac{\partial \theta}{\partial x_{j}}-\overline{\mu_{j}^{\prime} \theta^{\prime}}\right) \\
\frac{\partial_{C}}{\partial_{t}} \pm U_{j} \frac{\partial_{C}}{\partial_{x_{j}}}=\frac{\partial}{\partial x_{j}}\left(D \frac{\partial C}{\partial x_{j}}-\overline{\mu_{j}^{\prime} c^{\prime}}\right)
\end{gathered}
$$


The $\mu_{\mathrm{i}}$ and $\mathrm{x}_{\mathrm{i}}$ are the instantaneous velocity and the position, $\mathrm{p}$ the instantaneous pressure, $\mathrm{t}$ is the time, $\mathrm{Q}$ the fluid density, $v$ is the molecular kinematic viscosity, $c_{\mathrm{p}}$ heat capacity, $\mathrm{k}$ is the thermal conductivity and $\mathrm{S}_{\mathrm{ij}}$ the strain-rate tensor, $\mathrm{c}$ is the instantaneous concentration, and $\mathrm{D}$ is the molecular diffusion coefficient.

The Reynolds stresses component $\left(\overline{\mu_{j}{ }^{\prime} \mu_{i}}\right)$, describes the turbulence of the mean flow being the exchange of momentum by the change of the fluid parcels. In a laminar flow, the molecules are the responsible for momentum exchange (molecular viscosity). However, in a turbulent flow (turbulent viscosity) the parcels of flow are the ones that exchange the momentum. To finish this calculus, it is also necessary to use a turbulence model to represent flow scales. The first order Boussinessq eddy-viscosity hypothesis to model the Reynolds stress in function of velocity and time is used. However, it is also a necessary model for the linear or non-linear eddy-viscosity distinction. The Reynolds stress is given by:

$$
\overline{\mu_{j}^{\prime} \mu_{i}^{\prime}}=2 v_{t} S_{i j}-\frac{2}{3} k \delta_{i j}
$$

$\mathrm{v}_{\mathrm{t}}$ is the turbulent viscosity and the mean strain rate $\mathrm{S}_{\mathrm{ij}}$ is given by,

$$
S_{i j}=\frac{1}{2}\left(\frac{\partial U_{i}}{\partial_{x j}}+\frac{\partial j}{\partial_{x i}}\right)
$$

The turbulent kinetic energy $(\mathrm{k})$ is given by,

$$
k=\frac{1}{2} \overline{\mu_{i}^{\prime} \mu_{i}^{\prime}}
$$

And the Kronecker delta $\left(\delta_{\mathrm{ij}}\right)$,

$$
\delta_{i j}=\left\{\begin{array}{l}
1 ; \text { if } i=j \\
0 ; \text { if } i \neq j
\end{array}\right.
$$

Assuming the gradient diffusion (Gradient-diffusion Assumption) for heat and mass fluxes, as function of the temperature gradients in the mean flow, the turbulence hot flow is given by,

$$
-\overline{\mu_{j}^{\prime} \theta^{\prime}}=D_{\theta t} \frac{\partial \vartheta}{\partial_{x i}}
$$

$D_{\theta t}$ is the turbulent heat diffusivity (turbulent Prandtl number), and $\frac{\partial \vartheta}{\partial_{x i}}$ is the temperature gradient in the mean flow. The turbulent Prandtl number is obtained by, 


$$
P r_{t}=\frac{v_{t}}{D_{\theta, t}}
$$

And the turbulent mass flow $-\overline{\mu_{j}^{\prime} c^{\prime}}$ is given by,

$$
-\overline{\mu_{j}^{\prime} c^{\prime}}=D_{c, t} \frac{\partial C}{\partial x_{i}}
$$

$\mathrm{D}_{c, t}$ is the turbulent mass diffusivity (turbulent Schmidt number) and $\frac{\partial C}{\partial x_{i}}$ the concentrated gradient in the mean flow. The turbulent Schmidt number is calculated from Equation 36,

$$
S c_{t}=\frac{v_{t}}{D_{\theta, t}}
$$

Once RANS needed a turbulence model, less expensive equations are created with additional variables, transforming in meanings of the instantaneous equations calculations. This results from removing several small equations and adding other unknown variables, determined by the turbulence models. Standard $K-\varepsilon$ turbulence model used by [37] in a computational simulation on a counter-clock cyclist helmet, however, it is only valid in a completely turbulent fluid. The same mode is assumed for wheelchair racing. In FLUENT, the turbulence models available are: (i) Standard K - psilon; (ii) Standard K - $\varepsilon$; (iii) Spalart - Allmoras; (iv) Reynolds Stress (RSM) [38].

In this model (Standard $\mathrm{K}-\varepsilon$ ) the Bussinesq hypothesis is given by,

$$
-\rho \overline{\mu_{i}^{\prime} \mu_{j}^{\prime}}=2 \mu_{t} S_{i j}-\frac{2}{3} \rho k \delta_{i j}
$$

Where the turbulent viscosity,

$$
\mu_{t}=\rho C_{\mu} \frac{k^{2}}{\varepsilon}
$$

The mean tension rate is given by Equation 30. The kinetic energy of turbulent fluctuation and the dissipation of the kinetic energy $\left(\mathrm{m}^{2} / \mathrm{s}^{2}\right)$ are given by Equations 31 and 40 respectively.

$$
k=\frac{1}{2} \overline{\mu_{i}^{\prime} \mu_{i}^{\prime}}=\frac{1}{2} \overline{\mu^{\prime 2}}+\overline{v^{\prime 2}}+\overline{w^{\prime 2}}
$$


$\mathrm{k}$, is the energy measuring associated with the turbulent fluctuations in the flow.

$$
\varepsilon=v \frac{\overline{\partial \mu_{i}^{\prime} \partial \mu_{i}^{\prime}}}{\partial x_{j} \partial x_{j}}
$$

$\varepsilon$, is caused by the work of the smallest eddies against the viscous stresses in the flow.

Then the determination of $k$ (Equation 41) and $\varepsilon$ (Equation 42) by their transport equations are,

$$
\begin{gathered}
\rho \frac{\partial k}{\partial t}+\rho \frac{\partial}{\partial x_{i}}\left(k \mu_{i}\right)=\frac{\partial}{\partial x_{j}}\left(\left(\mu+\frac{\mu_{t}}{\sigma_{k}}\right) \frac{\partial k}{\partial x_{j}}\right)+G_{k}+G_{b}-\rho \varepsilon \\
\rho \frac{\partial \varepsilon}{\partial t}+\rho \frac{\partial}{\partial x_{i}}\left(\epsilon \mu_{i}\right)=\frac{\partial}{\partial x_{j}}\left(\left(\mu+\frac{\mu_{t}}{\sigma_{\varepsilon}}\right) \frac{\partial \varepsilon}{\partial x_{j}}\right)+C_{1 \varepsilon} \frac{\varepsilon}{k}\left(G_{k}+C_{3 \varepsilon} G_{b}\right)-\rho C_{2 \varepsilon} \frac{\varepsilon^{2}}{k}
\end{gathered}
$$

Where, $\rho \frac{\partial \varepsilon}{\partial t}$ and $\rho \frac{\partial k}{\partial t}$ are the variation of the local in time, $\rho \frac{\partial}{\partial x_{i}}\left(k \mu_{i}\right)$ and $\rho \frac{\partial}{\partial x_{i}}\left(\mu_{i}\right)$ the adjective term, $\frac{\partial}{\partial x_{j}}\left(\left(\mu+\frac{\mu_{t}}{\sigma_{k}}\right) \frac{\partial k}{\partial x_{j}}\right)$ and $\frac{\partial}{\partial x_{j}}\left(\left(\mu+\frac{\mu_{t}}{\sigma_{\varepsilon}}\right) \frac{\partial \varepsilon}{\partial x_{j}}\right)$ are the diffusion, $\mathrm{G}_{\mathrm{k}}$ is the generation of $\mathrm{k}$ by the gradients mean velocity, $G_{b}$ the generation of $k$ due the fluctuation and $\varrho \varepsilon$ the dissipation of k. $\sigma \mathrm{k}$ and $\sigma \varepsilon$ are the turbulent Prandtl numbers for $\mathrm{k}$ and $\varepsilon$ respectively. And the constants $\mathrm{C}_{1 \varepsilon}, \mathrm{C}_{2 \varepsilon}, \mathrm{C}_{\mu}, \sigma_{\mathrm{k}}$ e $\sigma_{\varepsilon}$ were experimentally determined, $\mathrm{C}_{1 \varepsilon}=1.44 ; \mathrm{C}_{2 \varepsilon}=1.92 ; \mathrm{C}_{\mu}=0.09 ; \sigma_{\mathrm{k}}=1.0$; $\sigma_{\varepsilon}=1.3$.

The Gambit software allows the building of a representative graphic model of the volume subdivided in sub-volumes, trying to make the process as realistic as possible. This software also allows defining the frontiers. In solid frontiers and close to them, the FLUENT software computes the Reynolds tension and $\varepsilon$. It applies solid specific frontiers conditions for Reynolds tension using balance hypotheses, without considering the convection and diffusion of tension transport (Equation 25). In a local coordinate system, $\mathrm{T}$ is tangential coordinate, $\eta$ the normal and $\lambda$ the binomial one. The Reynolds tension in the adjacent cells to the frontier, are calculated by the equation:

$$
\frac{\overline{\mu_{\mathrm{A}}^{\prime 2}}}{\mathrm{k}}=1.098, \frac{\overline{\mu_{\eta}^{\prime 2}}}{\mathrm{k}}=0.247, \frac{\overline{\mu^{\prime 2}}}{\mathrm{k}}=0.655, \frac{\overline{\mu_{\mathrm{t}}^{\prime} \mu_{\eta}^{\prime}}}{\mathrm{k}}=0.255
$$

FLUENT solves the transport equation (41) for k obtaining. For calculus convenience, the equation is globally solved, albeit the calculus of the $\mathrm{k}$ values is only necessary near the frontier. In the rest of the domain, the $\mathrm{k}$ is calculated by the equation (26).

The mesh can be constructed by quadrangular elements, with a space size of $0.1 \mathrm{~mm}$. The resulting data from the computational simulation of the determined flow regime and the visualization of the pressure profiles and speed are obtained by FLUENT. The data processing allows calculating the drag coefficient in the diverse forms [39]. 


\subsection{Numerical simulations on wheelchair racing}

CFD methodology starts being used for equipment tests. In wheeler riders' helmets, CFD has shown that airflow velocities could be improved with grooves in polymer foam liner and also improving the sweat evaporation. A top helmet hole would improve the velocity in that point, however velocities at the back became lower [40].

The ideal posture and some changes in the wheelchair could be possible to define the three first places. The tests revealed that the most drag negative influence in performance came from the athlete and not from the wheelchair. With a subtle modification in the sitting position, it could save $10 \%$ of the aerodynamic drag. No results were presented by the authors relatively to the wind tunnel test [41].

CFD methodology must be applied in different fabrics, helmets, and wheelchairs. The frame design, as the tube sizes must influence the fluid flow behavior. Also, different positions should be considered to be analyzed the flexed head in the start of the race or the look forward position. In each stroke phase, the different fluid flows should also be analyzed intending to reach the ideal motion in the stroke phase and recovery one, as the effect of the wheelchair designs and materials in the fluid flow [23].

Thus, fluid dynamics analysis should be performed intending to evaluate possible different designs of the wheelchairs and fulfill the lack of literature in this area. There is a need of results presentation such as drag and drag coefficient in different positions, helmets, and cloths at different speeds.

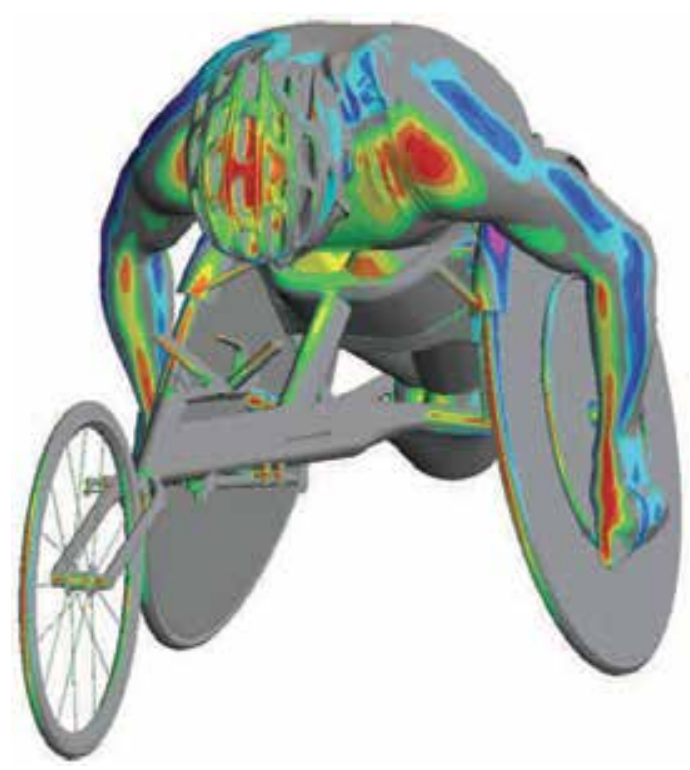

Figure 6. CFD Methodology of a Scanned Model in TotalSim (http://www.totalsimulation.co.uk/wp/cfd-image-gallery/) 


\section{Summary and conclusions}

The sports science of wheelchair racing lacks research. However, there is some information that could positively contribute to increase performance in athletes. Managing the mathematical models based on Physics laws, coaches could identify the mechanic performance obstacles and try to minimize them.

The drag reduction by the rolling and air resistance access also contributed to increase the performance in athletes. Better propulsion could be obtained by reducing the total drag combined with a mass reduction and other possible aerodynamic positions. The purpose of computational simulations and/or computer fluid dynamics is the time improvement achieved by reducing the aerodynamic drag. No data was found about CFD tests in wheelchair racing.

The stroke technique should also be focused, despite the lack of indications about the number of hours off training after an injury occurrence. It is defined that the high angular velocities near the shoulder and the elbow generated by the strokes induce an overuse stress increasing the risk for joint injuries. There are also some indications about the contact zones, force applying, and contact break at the rear wheels.

Physiological variables should also be studied in wheelchair racing athletes, an area that lacks information in this sport. Thus, a precise control of the total drag and the efficiency of the stroke technique, which is related to high levels of strength derived by the strength and physical condition training, will positively contribute for a better performance.

\section{Author details}

Pedro Forte ${ }^{1,2}$, Tiago M. Barbosa ${ }^{2,3}$ and Daniel A. Marinho ${ }^{1,2^{*}}$

*Address all correspondence to: dmarinho@ubi.pt

1 University of Beira Interior, Department of Sport Sciences, Covilhã, Portugal

2 Research Centre in Sports, Health and Human Development, Covilhã, Portugal

3 National Institute of Education. Nanyang Technological University, Singapore

\section{References}

[1] IWAS (2008) IWAS Athletics, worldwide ranking. http://athletics.iwasf.com/. Accessed 14 Aug 2009

[2] IPC International Paralympic Committee (2007) IPC athletics classification manual for physical impairments 2008-2010. http://www.paralympic.org/release/Summ- 
er_Sports/Athletics/News/2007_12_07_ClassificationManual.pdf. Accessed 14 Aug 2009.

[3] Depauw, K. P. (1986). Research on sport for athletes with disabilities. Adap Phys Activ Q, 3(4): 292-299.

[4] Depauw, K. P. (1988). Sports for individuals with disabilities: Research opportunities. Adap Phys Activ Q, 5: 80-89.

[5] Cooper, R. A. (1990c). Wheelchair racing sports science: a review. Journal of rehabilitation research and development, 27(3), 295-312.

[6] Lakomy, H. K. A., Cambell, I. \& Williams, C. (1987). Treadmill performance and selected physiological characteristics of wheelchair athletes. Br J Sports Med, 21(3), 130-133.

[7] Ridgway, M., Pope, C., Wilkerson, J. (1988). A kinematic analysis of 800-meter wheelchair racing techniques. Adapt Phys Act Q 5(2): 96-107.

[8] Bymes, D.P. (1983). APlm and EMG drive phase analysis of the competitive wheelchair stroke. Unpublished master's thesis, University of Alberta.

[9] Cooper, R. A. (1990b) An exploratory study of racing wheelchair propulsion dynamics. Adapt Phys Act Q, 7(1): 74-85.

[10] Sanderson, D. J., Sommer, H. J. (1985). Kinematic features of wheelchair propulsion. J Biomech 18(6): 423-429.

[11] Cooper, R. A. (1990). A systems approach to the modeling of racing wheelchair propulsion. J Rehabil Res Dev, 27(2): 151-62.

[12] Davis, R., Ferrara, M., \& Byrnes, D. (1988). The competitive wheelchair stroke. NSCA Journal, 10(3), 4-10.

[13] Higgs, C. (1985). Propulsion of racing wheelchairs. In C. Sherrill (Ed.), Sport and disabled athletes (pp. 165-172). Champaign, IL: Human Kinetics

[14] Chow, J. W., Chae, W. (2007). Kinematic analysis of the $100 \mathrm{~m}$ wheelchair race. J Biomech, 40: 2564-2568.

[15] Costa, G. B., Rubio, M. P., Belloch, S. L., \& Soriano, P. P. (2009). Case study: effect of handrim diameter on performance in a paralympic wheelchair athlete.Adapted physical activity quarterly, 26(4), 352-363.

[16] O'Connor, T., Robertson, R. N., Cooper, R. A. (1998). Three-dimensional kinematic analysis and physiologic assessment of racing wheelchair propulsion. Adapt Phys Activ Q, 15: 1-14.

[17] Keogh, J. W. L. (2011). Paralympic sport: an emerging area for research and consultancy in sports biomechanics. Sports Biomech, 10: 249-268. 
[18] Rudins, A., Laskowski, E. R., Growney, E. S., Cahalan, T. D., \& An, K. N. (1997). Kinematics of the elbow during wheelchair propulsion: a comparison of two wheelchairs and two stroking techniques. Archives of physical medicine and rehabilitation, 78(11), 1204-1210.

[19] Gehlsen, G. M., Davis, R. W., \& Bahamonde, R. (1990). Intermittent velocity and wheelchair performance characteristics. Adapted Physical Activity Quarterly, 7(3), 219-230.

[20] Coutts, K. D. (1990). Kinematics of sport wheelchair propulsion. Journal of rehabilitation research and development, 27(1), 21-26.

[21] Fuss, F. K. (2009). Influence of mass on the speed of wheelchair racing. Sports Engineering, 12(1): 41-53.

[22] LaMere, T.J., \& Labanowich, S. (1984). The history of sports wheelchairs: Part III, The racing wheelchair 1976-1983. Sports 'n Spokes, 10 (1), 12-16.

[23] Barbosa, T. M., Forte, P., Morais, J. E., Coelho, E. (2014). Partial contribution of rolling friction and drag force to total resistance of an elite wheelchair athlete. 1st International Conference in Sports Sciences \& Technology. ICSST, Advanced Materials for Sport Technology, 749-753.

[24] Burke, E. (Ed.). (1986). Science of cycling. Champaign, IL: Human Kinetics Publishers.

[25] Hedrick, B., Wang, Y. T., Moeinzadeh, M., \& Adrian, M. (1990). Aerodynamic positioning and performance in wheelchair racing. Adapted Physical Activity Quarterly, $7(1)$.

[26] FIFA. (2001). Quality Concept for Football Turf. Available: http://www.fifa.com/mm/ document/afdeveloping/pitchequip/fqc_football_turf_folder_342.pdf.

[27] Kolitzus, H. J. (2003). Ball Roll Behavior: The Functional Relationship of the Ball Roll Distance and the Timing Gate Method; How to Calculate the Ball Roll Distance from Timing Gate Measurements. ISSS - International Association for Sports Surface Sciences, Eschenz / Switzerland, Available: http://www.isss-sportsurfacescience.org/ downloads/documents/ZPKPAJJUWY_Ball_Roll_BehaviorKS.pdf.

[28] Kwarciak, A. M., Yarossi, M., Ramanujam, A., Dyson-Hudson, T. A., \& Sisto, S. A. (2009). Evaluation of wheelchair tire rolling resistance using dynamometer-based coast-down tests. J Rehabil Res Dev, 46(7), 931-38.

[29] DiGiovine CP, Cooper RA, Boninger ML. Dynamic calibration of a wheelchair dynamometer. J Rehabil Res Dev. 2001;38(1):41-55.

[30] Candau, R. B., Grappe, F., Menard, M., Barbier, B., Millet, G.Hoffman, M. D. 1999. Simplified deceleration method for assessment of resistive forces in cycling. Medicine and Science in Sports and Exercise, 31: 1441-1447. 
[31] MacLeish, M. S., Cooper, R. A., Harralson, J., \& Ster, J. F. (1993). Design of a composite monocoque frame racing wheelchair. Journal of rehabilitation research and development, 30, 233-233.

[32] Burton, M., Fuss, F. K., \& Subic, A. (2010). Sports wheelchair technologies. Sports Technology, 3(3), 154-167.

[33] Petrushov, V. A. (1998). Improvement in vehicle aerodynamic drag and rolling resistance determination from coast-down tests. Proceedings of the Institution of Mechanical Engineers, Part D: Journal of Automobile Engineering, 212(5), 369-380.

[34] Hanna, R. K. (2012). CFD in Sport-a Retrospective; 1992-2012. Procedia Engineering, $34,622-627$.

[35] Marinho, D. A., Barbosa, T. M., Mantha, V., Rouboa, A. I., \& Silva, A. J. (2012). Modelling propelling force in swimming using numerical simulations. In: Juarez LH (Ed). Fluid Dynamics, Computational Modeling and Applications. Pp. 439-448. InTech. Rijeka.

[36] Marinho, D.A., Silva, A.J., Reis, V.M., Barbosa, T. M., Vilas-Boas, J.P., Alves, F.B., Machado, L., Rouboa, A. (2011) Three-dimensional CFD analysis of the hand and forearm in swimming. Journal of Applied Biomechanics. ISSN 1065-8483. 27:1, p. 74-80.

[37] Caboz, E. B. (2010). Simulação computacional do escoamento em torno de um capacete de ciclista usado nas provas de contra-relógio. Dissertação de mestrado. Faculdade de Engenharia da Universidade do Porto.

[38] White, F. (1999). Mecanica dos Fluidos, Ed. McGraw -Hill, 4⿳⺈ edicao. ISBN : 85-86804-24-X.

[39] Carvalho, F. (2008). A aplicação da dinâmica computacional de fluidos no estudo do arrasto hidrodinâmico na natação. Dissertação de Mestrado em Educação Fisica e Desporto. Universidade de Trás os Montes. Vila Real.

[40] Pinnoji, P. K., \& Mahajan, P. (2006, September). Impact analysis of helmets for improved ventilation with deformable head model. In Proceeding of IRCOBI conference, Madrid (pp. 159-70).

[41] Rushby-Smith, T., \& Douglas, L. (2012). Paralympic Technology. Ingenia, 51, p.33. 


